\title{
ON THE INTEGRAL HODGE CONJECTURE FOR REAL VARIETIES, I
}

\author{
OLIVIER BENOIST AND OLIVIER WITTENBERG
}

\begin{abstract}
We formulate the "real integral Hodge conjecture", a version of the integral Hodge conjecture for real varieties, and raise the question of its validity for cycles of dimension 1 on uniruled and Calabi-Yau threefolds and on rationally connected varieties. We relate it to the problem of determining the image of the Borel-Haefliger cycle class map for 1-cycles, with the problem of deciding whether a real variety with no real point contains a curve of even geometric genus and with the problem of computing the torsion of the Chow group of 1-cycles of real threefolds. New results about these problems are obtained along the way.
\end{abstract}

\section{INTRODUCTION}

One of the central problems in the study of algebraic cycles of codimension $k$ on a smooth proper complex algebraic variety $X$ consists in determining the subgroup $H_{\text {alg }}^{2 k}(X(\mathbf{C}), \mathbf{Z}) \subseteq H^{2 k}(X(\mathbf{C}), \mathbf{Z})$ formed by their cycle classes. Hodge theory provides a chain of inclusions

$$
H_{\text {alg }}^{2 k}(X(\mathbf{C}), \mathbf{Z}) \subseteq \operatorname{Hdg}^{2 k}(X(\mathbf{C}), \mathbf{Z}) \subseteq H^{2 k}(X(\mathbf{C}), \mathbf{Z}),
$$

where $\operatorname{Hdg}^{2 k}(X(\mathbf{C}), \mathbf{Z})$ denotes the set of those classes whose images in $H^{2 k}(X(\mathbf{C}), \mathbf{C})$ have type $(k, k)$ with respect to the Hodge decomposition. By the Hodge conjecture, the first inclusion should become an equality after tensoring with $\mathbf{Q}$. It is customary to refer to the property that the first inclusion is itself an equality as the integral Hodge conjecture. Despite its name, this property can fail. Its study for specific $X$ and $k$ has nevertheless played a significant role in recent years (see [Voi06], [CTV12], [Voi14, Chapter 6] and the references therein, and \$2.1for a more detailed discussion).

Let now $X$ denote a smooth proper real algebraic variety, by which we mean a smooth proper scheme over $\mathbf{R}$. With any algebraic cycle of codimension $k$ on $X$, Borel and Haefliger [BH61 have associated a cycle class in $H^{k}(X(\mathbf{R}), \mathbf{Z} / 2 \mathbf{Z})$. The study of the subgroup $H_{\text {alg }}^{k}(X(\mathbf{R}), \mathbf{Z} / 2 \mathbf{Z}) \subseteq H^{k}(X(\mathbf{R}), \mathbf{Z} / 2 \mathbf{Z})$ formed by these classes is a classical topic in real algebraic geometry (see [Sil89, Chapter III], [BCR98, §11.3], [BK98, Man17, Chapitres 3-4] and the references therein), related to the problem of $\mathscr{C}^{\infty}$ approximation of submanifolds of $X(\mathbf{R})$ by algebraic subvarieties.

Despite the formal similarity between these two settings, one critical difference stands out: being expressed with torsion coefficients, the definition of the subgroup

Date: January 2nd, 2018; revised on April 30th, 2019. 
$H_{\mathrm{alg}}^{k}(X(\mathbf{R}), \mathbf{Z} / 2 \mathbf{Z})$ misses any information that might come from the Hodge theory of the underlying complex variety. The latter, however, does have an influence on this subgroup (see, e.g., [vH00a, Chapter IV, Corollary 4.4] or [Man97]).

The main aim of the present work is to put forward and examine the real integral Hodge conjecture, a statement for real algebraic varieties which is analogous to the (complex) integral Hodge conjecture recalled above and whose study refines, at the same time, that of $H_{\text {alg }}^{k}(X(\mathbf{R}), \mathbf{Z} / 2 \mathbf{Z})$. In part I, we formulate it (\$2) and, focusing on the case of 1-cycles, study its consequences (\$3, \$5) while part II (that is, [BW18]) establishes particular cases of it, again for 1-cycles (see [BW18, §6, §7, §8, §§9.4-9.5]).

More specifically, we define, in \$2, a subgroup

$$
\operatorname{Hdg}_{G}^{2 k}(X(\mathbf{C}), \mathbf{Z}(k))_{0} \subseteq H_{G}^{2 k}(X(\mathbf{C}), \mathbf{Z}(k)),
$$

where $G=\operatorname{Gal}(\mathbf{C} / \mathbf{R})$ acts on the space $X(\mathbf{C})$ and on the group $\mathbf{Z}(k)=(\sqrt{-1})^{k} \mathbf{Z}$ and where $H_{G}^{2 k}(X(\mathbf{C}), \mathbf{Z}(k))$ denotes $G$-equivariant cohomology in the sense of Borel, by combining the Hodge condition in $H^{2 k}(X(\mathbf{C}), \mathbf{C})$ with a topological condition in $H_{G}^{2 k}(X(\mathbf{R}), \mathbf{Z}(k))$ discovered by Kahn and Krasnov [Kah87, Kra94. When $k \leq 1$, this topological condition is trivial; when $\operatorname{dim}(X) \leq 3$ and $k=2$, it is simply the requirement that the pull-back to any real point of $X$ of the equivariant cohomology class under consideration should vanish.

We prove that the subgroup (10.2) is compatible with cup products, pull-backs and proper push-forwards (see \$1.6.4 and Theorem 1.21). It contains the subgroup of $H_{G}^{2 k}(X(\mathbf{C}), \mathbf{Z}(k))$ formed by the equivariant cycle classes of algebraic cycles of codimension $k$. The real integral Hodge conjecture refers to the property that every element of (10.2) is the equivariant cycle class of some algebraic cycle of codimension $k$. Just as in the complex situation, the real integral Hodge conjecture sometimes fails, though it always holds when $k=1$ (an observation due to Krasnov) or $k=\operatorname{dim}(X)$ (see \$2.3.2), it is a birational invariant when $k=2$ or $k=\operatorname{dim}(X)-1$ (see \$2.3.3), and it holds for projective spaces (see \$2.3.4).

As was noted by Krasnov Kra94 and by van Hamel vH00a, there exists a canonical map

$$
H_{G}^{2 k}(X(\mathbf{C}), \mathbf{Z}(k)) \rightarrow H^{k}(X(\mathbf{R}), \mathbf{Z} / 2 \mathbf{Z})
$$

which sends the equivariant cycle class of any codimension $k$ algebraic cycle to its Borel-Haefliger cycle class. Considering the image $H$ of $\operatorname{Hdg}_{G}^{2 k}(X(\mathbf{C}), \mathbf{Z}(k))_{0}$ by this map now leads to a chain of inclusions analogous to (0.1):

$$
H_{\text {alg }}^{k}(X(\mathbf{R}), \mathbf{Z} / 2 \mathbf{Z}) \subseteq H \subseteq H^{k}(X(\mathbf{R}), \mathbf{Z} / 2 \mathbf{Z})
$$

Obviously, if $H \neq H^{k}(X(\mathbf{R}), \mathbf{Z} / 2 \mathbf{Z})$, then $H_{\mathrm{alg}}^{k}(X(\mathbf{R}), \mathbf{Z} / 2 \mathbf{Z}) \neq H^{k}(X(\mathbf{R}), \mathbf{Z} / 2 \mathbf{Z})$. This implication already explains all of the known examples of real varieties $X$ such that $H_{\text {alg }}^{k}(X(\mathbf{R}), \mathbf{Z} / 2 \mathbf{Z}) \neq H^{k}(X(\mathbf{R}), \mathbf{Z} / 2 \mathbf{Z})$ for some $k$ (see Remark $\left.2.7(\mathrm{ii})\right)$. In $\$ 4.3$, 
we provide an example that cannot be explained by this mechanism. It is based on a degeneration argument to positive characteristic, as in [Tot13], to contradict the real integral Hodge conjecture.

Unlike the equality $H_{\mathrm{alg}}^{k}(X(\mathbf{R}), \mathbf{Z} / 2 \mathbf{Z})=H^{k}(X(\mathbf{R}), \mathbf{Z} / 2 \mathbf{Z})$, the real integral Hodge conjecture turns out to be an interesting property when $X(\mathbf{R})=\varnothing$ as well. When $X(\mathbf{R})=\varnothing$ and $X$ has pure dimension $d$, we construct a canonical map

$$
H_{G}^{2 d-2}(X(\mathbf{C}), \mathbf{Z}(d-1)) \rightarrow \mathbf{Z} / 2 \mathbf{Z}
$$

which sends the equivariant cycle class of a reduced curve $Z \subseteq X$ with normalisation $Z^{\prime}$ to $\chi\left(Z^{\prime}, \mathscr{O}_{Z^{\prime}}\right)$ modulo 2 (see Theorem [3.6). In particular, the equivariant cycle class of a geometrically irreducible curve on $X$ determines its geometric genus modulo 2, whereas the other cycle classes we have mentioned do not. Considering the image of $\operatorname{Hdg}_{G}^{2 d-2}(X(\mathbf{C}), \mathbf{Z}(d-1))_{0}$ by this map now provides a possible obstruction to the existence of a geometrically irreducible curve of even geometric genus on $X$, and, in particular, to the existence of a geometrically rational curve. We give examples of this in $\$ \$ 4.14 .2$. We note that any smooth proper and geometrically irreducible variety of dimension $\geq 2$ contains a geometrically irreducible curve of odd geometric genus (see Proposition [3.9); the existence of a geometrically irreducible curve of even geometric genus is a property which does not seem to have been considered systematically before (though see [Kol13, Example 41, Question 42]).

Let $\psi$ denote the restriction of the map (0.3) for $k=d-1$, if $X(\mathbf{R}) \neq \varnothing$, or of the map (0.5), if $X(\mathbf{R})=\varnothing$, to the subgroup

$$
H_{G}^{2 d-2}(X(\mathbf{C}), \mathbf{Z}(d-1))_{0} \subseteq H_{G}^{2 d-2}(X(\mathbf{C}), \mathbf{Z}(d-1))
$$

cut out by the topological condition which enters the definition of (0.2) (disregarding the Hodge condition). To draw consequences of the real integral Hodge conjecture for 1-cycles, one quickly faces the problem of determining the image of $\psi$. Using a new result of a purely topological nature established in \$1 (see Theorem 1.12), we solve it completely in \$3 (see Theorem [3.3), thus providing an answer to a question of van Hamel (see [H00a, p. 93], where the map $\rho_{1}$ is our $(\underline{0.3})$ for $k=d-1$ ).

When the 2-torsion subgroup of $\operatorname{Pic}\left(X_{\mathbf{C}}\right)$ is trivial (e.g., when $X(\mathbf{C})$ is simply connected), the map $\psi$ is surjective and we show that its kernel consists of norms from $\mathbf{C}$ to $\mathbf{R}$ of classes in $H^{2 d-2}(X(\mathbf{C}), \mathbf{Z})$. A direct relation follows, in this case, between four motifs: the real integral Hodge conjecture, the complex integral Hodge conjecture, the surjectivity of the Borel-Haefliger cycle class map and the existence of geometrically irreducible curves of even geometric genus (see Theorem 3.22).

Two theorems that we obtain as consequences of these results - in conjunction, in the case of Theorem $\mathrm{B}$, with Voisin's theorem according to which complex uniruled or Calabi-Yau threefolds satisfy the integral Hodge conjecture (see [Voi06]) - are 
the following. If $M$ is an abelian group, we denote by $M\left[2^{\infty}\right]$ its 2 -primary torsion subgroup.

Theorem A (see Theorem 3.16). Let X be a smooth, proper and geometrically irreducible real variety, of dimension $d \geq 1$. Assume that $X$ satisfies the real integral Hodge conjecture for 1 -cycles and that $H^{2}\left(X, \mathscr{O}_{X}\right)=0$.

(i) The subgroup $H_{\text {alg }}^{d-1}(X(\mathbf{R}), \mathbf{Z} / 2 \mathbf{Z}) \subseteq H^{d-1}(X(\mathbf{R}), \mathbf{Z} / 2 \mathbf{Z})$ is the exact orthogonal complement, under the Poincaré duality pairing, of the image of $\operatorname{Pic}(X)\left[2^{\infty}\right]$ by the Borel-Haefliger cycle class map $\operatorname{Pic}(X) \rightarrow H^{1}(X(\mathbf{R}), \mathbf{Z} / 2 \mathbf{Z})$.

(ii) There exists a geometrically irreducible curve of even geometric genus in $X$ if and only if the natural map $\operatorname{Pic}(X)\left[2^{\infty}\right] \rightarrow \operatorname{Pic}\left(X_{\mathbf{C}}\right)^{G}\left[2^{\infty}\right]$ is onto.

Theorem $\mathrm{A}$ applies, in particular, to surfaces of geometric genus zero. Even in this case, its conclusions are new, except for (i) when $X_{\mathbf{C}}$ is a surface of geometric genus zero such that $\operatorname{Pic}\left(X_{\mathbf{C}}\right)[2]=0$ (Silhol, van Hamel; see [Man17, Théorème 3.7.18]), an Enriques surface (Mangolte and van Hamel [MvH98]) or a birationally ruled surface (Kucharz Kuc01]). Among the new corollaries of Theorem A, we find that any real Enriques surface contains a geometrically irreducible curve of even geometric genus and that any real surface of geometric genus zero such that $H^{1}(X(\mathbf{R}), \mathbf{Z} / 2 \mathbf{Z}) \neq 0$ satisfies $H_{\text {alg }}^{1}(X(\mathbf{R}), \mathbf{Z} / 2 \mathbf{Z}) \neq 0$ (see 3.6 .2$)$.

Theorem B (see Corollary 3.23). Let $X$ be a smooth and proper real threefold. Assume that $X_{\mathbf{C}}$ is rationally connected or is simply connected Calabi-Yau. Then the real integral Hodge conjecture for $X$ is equivalent to the equality $H_{\mathrm{alg}}^{2}(X(\mathbf{R}), \mathbf{Z} / 2 \mathbf{Z})=$ $H^{2}(X(\mathbf{R}), \mathbf{Z} / 2 \mathbf{Z})$, if $X(\mathbf{R}) \neq \varnothing$, or to the existence of a geometrically irreducible curve of even geometric genus on $X$, if $X(\mathbf{R})=\varnothing$.

As Theorem B clearly illustrates, the existence of a geometrically irreducible curve of even geometric genus must be considered as the analogue, in the absence of real points, of the equality $H_{\mathrm{alg}}^{d-1}(X(\mathbf{R}), \mathbf{Z} / 2 \mathbf{Z})=H^{d-1}(X(\mathbf{R}), \mathbf{Z} / 2 \mathbf{Z})$.

Over $\mathbf{C}$, Voisin has proved the integral Hodge conjecture for 1-cycles on uniruled or Calabi-Yau threefolds and, conditionally on the Tate conjecture for surfaces over finite fields, on rationally connected varieties of any dimension (see [Voi06], [Voi13]). The analogy between the real and complex integral Hodge conjectures, on the one hand, and the good properties of the real integral Hodge conjecture, on the other hand, prompt the following question, which serves as a guiding problem for [BW18]: if $X_{\mathbf{C}}$ is a uniruled threefold, a Calabi-Yau threefold, or a rationally connected variety, does $X$ satisfy the real integral Hodge conjecture for 1-cycles?

By Theorem $\mathrm{A}$, a positive answer would imply that rationally connected varieties (of positive dimension) over $\mathbf{R}$ satisfy $H_{\mathrm{alg}}^{d-1}(X(\mathbf{R}), \mathbf{Z} / 2 \mathbf{Z})=H^{d-1}(X(\mathbf{R}), \mathbf{Z} / 2 \mathbf{Z})$ and contain geometrically irreducible curves of even geometric genus. A conjecture of 
Kollár predicts that such varieties should even contain geometrically rational curves (see [AK03, Remarks 20], [Kol13, Question 42]).

In BW18, we provide evidence towards a positive answer to the above question by establishing the real integral Hodge conjecture for 1-cycles on $X$ under any of the following assumptions:

(1) $X$ is a conic bundle over a variety which itself satisfies the real integral Hodge conjecture for 1-cycles (e.g., $X$ can be any conic bundle threefold);

(2) $X$ is a Fano threefold with no real point;

(3) $X$ is a threefold fibred over a curve into del Pezzo surfaces of degree $\delta$, when $\delta \notin\{1,2,4\}$, as well as in some cases for which $\delta \in\{1,2,4\}$ (see [BW18] for precise statements).

In view of Theorem $\mathrm{A}$, these results have concrete consequences. Among them:

(i) the existence of a geometrically irreducible curve of even geometric genus in any smooth real quartic threefold;

(ii) the equality $H_{\mathrm{alg}}^{2}(X(\mathbf{R}), \mathbf{Z} / 2 \mathbf{Z})=H^{2}(X(\mathbf{R}), \mathbf{Z} / 2 \mathbf{Z})$ when $X$ is the total space of a fibration into cubic surfaces over a real curve $B$ such that $B(\mathbf{R})$ is connected.

The determination of the subgroup $H_{\mathrm{alg}}^{k}(X(\mathbf{R}), \mathbf{Z} / 2 \mathbf{Z})$ also has consequences on the problem of $\mathscr{C}^{\infty}$ approximation of submanifolds of $X(\mathbf{R})$ by algebraic subvarieties. As an example, combining (ii) with a result of Akbulut and King [AK88] (see also BK03] and [BW18, §6.2]) shows that for any threefold $X$ as in (ii), any $\mathscr{C}^{\infty}$ loop in $X(\mathbf{R})$ can be approximated arbitrarily well by the real locus of an algebraic curve.

Finally, we examine, in \$5, the implications of the real integral Hodge conjecture for the study of torsion 1-cycles on real varieties. We obtain, in particular, the following theorem. Its proof relies, on the one hand, on Bloch-Ogus theory, which we develop further in \$5 for real varieties, and, on the other hand, on the topological result already mentioned above (Theorem 1.12).

Theorem C (see Corollary 5.5). Let $X$ be a smooth, proper and geometrically irreducible real variety, of dimension d. Assume that $\mathrm{CH}_{0}\left(X_{\mathbf{C}}\right)$ is supported on a surface (such is the case, for instance, if $X_{\mathbf{C}}$ is a uniruled threefold) and that $H^{2}\left(X, \mathscr{O}_{X}\right)=0$. If $X$ satisfies the real integral Hodge conjecture for 1-cycles, then the image of $\mathrm{CH}_{1}(X)\left[2^{\infty}\right]$ by the Borel-Haefliger cycle class map

$$
\mathrm{CH}_{1}(X) \rightarrow H^{d-1}(X(\mathbf{R}), \mathbf{Z} / 2 \mathbf{Z})
$$

is the exact orthogonal complement, under the Poincaré duality pairing, of the subgroup $H_{\text {alg }}^{1}(X(\mathbf{R}), \mathbf{Z} / 2 \mathbf{Z}) \subseteq H^{1}(X(\mathbf{R}), \mathbf{Z} / 2 \mathbf{Z})$.

Theorem $\mathrm{A}$ (i) and Theorem $\mathrm{C}$ coincide in the case of surfaces.

For real threefolds $X$ which satisfy the real integral Hodge conjecture, Bloch-Ogus theory also enables us to control the torsion subgroup of the kernel of the equivariant cycle class map $\mathrm{CH}_{1}(X) \rightarrow H_{G}^{2 d-2}(X(\mathbf{C}), \mathbf{Z}(d-1))$. As an example, we prove in $\$ 5$ 
that for any smooth real quartic threefold $X$ with no real point, the abelian group $\mathrm{CH}_{1}(X)_{\text {tors }}$ is isomorphic to $\mathbf{Z} / 2 \mathbf{Z} \oplus(\mathbf{Q} / \mathbf{Z})^{30}$ (see Proposition [5.7, one can even determine the full structure of $\mathrm{CH}_{1}(X)$, see Remark 5.10 (i)). This relies on the real integral Hodge conjecture for such $X$, which we establish in BW18.

In this article as well as in [BW18], we work over an arbitrary real closed field, except when we use the specific archimedean properties of $\mathbf{R}$, via the StoneWeierstrass theorem as in [BW18, §6] or via Hodge theory as in [BW18, §7]. The statements of Theorems A, B, C, established in the present article, remain true in this generality, mutatis mutandis, while some of the results proved in [BW18] do not. As is well known, and as we recall in [BW18, §9.1], the truth, over an arbitrary real closed field, of an assertion such as the equality $H_{\mathrm{alg}}^{d-1}(X(\mathbf{R}), \mathbf{Z} / 2 \mathbf{Z})=H^{d-1}(X(\mathbf{R}), \mathbf{Z} / 2 \mathbf{Z})$ or the existence of a geometrically irreducible curve of even geometric genus in $X$ is equivalent to the truth of the same assertion over the reals together with a bound, in any bounded family of real varieties, on the degree of the curves whose existence it predicts. Thus, for instance, it follows from Theorem 3.16 (which is Theorem A over a real closed field) that in any bounded family of geometrically rational surfaces $X$, every $\mathscr{C}^{\infty}$ loop in $X(\mathbf{R})$ is homologically equivalent to the real locus of an algebraic curve of bounded degree. For rationally connected threefolds, however, the same is not true, as the real integral Hodge conjecture can fail for them over non-archimedean real closed fields (see [BW18, §9]). This is in marked contrast with the situation over algebraically closed fields of characteristic 0: by the Lefschetz principle, Voisin's theorem on the integral Hodge conjecture for rationally connected threefolds readily extends to such fields.

The text is organised as follows. We devote $\$ 1$ to the cohomological tools that are used throughout the article (both reminders and new results); we refer the reader to the introduction of $\$ 1$ for more details. In \$2, we formulate the real integral Hodge conjecture, prove a few basic results about it (e.g., its birational invariance for cycles of dimension 1 or of codimension 2) and raise the question of its validity for rationally connected varieties, uniruled threefolds and Calabi-Yau threefolds over $\mathbf{R}$. We proceed, in \$3, to relate the real integral Hodge conjecture for 1-cycles to the study of the group $H_{\mathrm{alg}}^{d-1}(X(\mathbf{R}), \mathbf{Z} / 2 \mathbf{Z})$ and of geometrically irreducible curves of even geometric genus. This leads us, in particular, to Theorems $\mathrm{A}$ and $\mathrm{B}$. A number of examples of smooth, proper and geometrically irreducible varieties $X$ over $\mathbf{R}$ such that $H_{\mathrm{alg}}^{d-1}(X(\mathbf{R}), \mathbf{Z} / 2 \mathbf{Z}) \neq H^{d-1}(X(\mathbf{R}), \mathbf{Z} / 2 \mathbf{Z})$ or such that $X$ does not contain any geometrically irreducible curve of even geometric genus are presented in \$4. Finally, we develop Bloch-Ogus theory and apply the real integral Hodge conjecture to the study of torsion 1-cycles in \$5, where we prove, in particular, Theorem C,

Acknowledgements. Krasnov and van Hamel were the first to approach algebraic cycles on real varieties in a systematic way through the study of the cycle class map 
into the equivariant integral singular cohomology of the complex locus. We would like to emphasise the importance of their work to the development of the subject considered in this article. In addition, we thank the referee for their careful work and for many suggestions which helped improve the exposition.

\section{Cohomology of Real algebraic varieties}

We introduce, in this section, the cohomological tools on which this article and its sequel [BW18] heavily depend. Some of these tools are standard (at least for varieties over the field $\mathbf{R}$ of real numbers), while some are new. Let us describe the organisation of \$1.

For lack of an adequate reference to the existing literature, we first recall, in \$1.1, the formalism of sheaf cohomology and equivariant sheaf cohomology for algebraic varieties over a real closed field, together with some of the standard properties that we shall use throughout: the two spectral sequences of equivariant cohomology; Poincaré duality à la Verdier; purity, equivariant purity; covariant functoriality. Over the field of real numbers, these topics are discussed in [vH00a, Chapter II and Chapter III]. Over a real closed field, one has to replace singular cohomology with semi-algebraic cohomology, a theory first developed by Delfs and Knebusch (see [DK82a], Del91]).

Letting $G=\mathbf{Z} / 2 \mathbf{Z}$, the $G$-equivariant cohomology groups of a space endowed with the trivial action of $G$, with coefficients in $\mathbf{Z} / 2 \mathbf{Z}$, in $\mathbf{Z}$, or in the twisted integers $\mathbf{Z}(1)$, canonically decompose as direct sums of non-equivariant cohomology groups. These decompositions appear in [Kah87], Kra94], vH00a, Chapter III, \$\$6-7]. They play an essential role in the formulation of the real integral Hodge conjecture (see \$1.6.3 and \$2.2). We discuss them in \$1.2, in the setting of semi-algebraic cohomology and with locally constant sheaves as coefficients.

In $\$ 1.3$, we show that in the case of the complex locus of a smooth real algebraic variety with support in the real locus, the long exact sequence of equivariant cohomology with support decomposes into canonically split short exact sequences, when one works with $\mathbf{Z} / 2 \mathbf{Z}$ coefficients (Proposition 1.3) or with appropriately twisted integer coefficients (Proposition [1.7). Proposition [1.3 is an improvement on [vH00b, §2.2]; Proposition 1.7 and its companion Proposition 1.8, however, seem to be entirely new. The results of $\$ 1.3$ are used in \$1.4, in \$5] and in [BW18, proof of Theorem 6.1, Step 4].

The goal of $\$ 1.4$ is to formulate and prove Theorem 1.12, a duality result which combines, for any smooth and proper real variety, Poincaré duality for the real locus with Lefschetz duality for the complement of the real locus in the complex locus. Theorem 1.12 is new, and is key to the proofs of Theorem 3.3 and Theorem 5.4.

In \$1.5, we establish a Lefschetz hyperplane theorem for equivariant cohomology with twisted integral coefficients (Proposition 1.15). This will be used in [BW18, proof of Theorem 7.1, §7.3]. 
Finally, we devote $\$ 1.6$ to the equivariant cycle class map associated with a smooth real algebraic variety, and to the topological constraint discovered by Kahn [Kah87] and Krasnov [Kra94 that all algebraic cycle classes must satisfy (see Theorem 1.18). A new result here is Theorem 1.21, which asserts the compatibility of this topological constraint with proper push-forwards. Its proof rests on the contents of $\$ 1.3$ and on a relative version of Wu's theorem due to Atiyah and Hirzebruch. Theorem 1.21 is used in \$2.3.3 and in [BW18, proofs of Theorem 6.1 and Theorem 8.1 (iv)].

In the whole article, in an effort to keep the notation as tidy as possible (especially in the equivariant setting), we stick to cohomology and do not introduce Borel-Moore homology. For smooth equidimensional varieties, this makes no difference: if $\mathscr{F}$ is a locally constant sheaf of abelian groups on an equidimensional semi-algebraic space $V$ subject to the assumptions of $\$ 1.1 .4$ below, one could regard $H_{i}^{\mathrm{BM}}(V, \mathscr{F})$ as shorthand for $H^{\operatorname{dim}(V)-i}\left(V, \mathscr{F} \otimes_{\mathbf{Z}}\right.$ or $\left._{V}\right)$, where or $_{V}$ denotes the orientation sheaf of $V$ (see [Del91, Chapter III, §9, Theorem 9.3]). Some of the results below (e.g., Theorem [3.6) would extend to singular varieties if one replaced cohomology with Borel-Moore homology.

1.1. Sheaf cohomology over real closed fields. We fix a real closed field $R$ and set $C=R(\sqrt{-1}), G=\operatorname{Gal}(C / R)$ and $\mathbf{Z}(j)=(\sqrt{-1})^{j} \mathbf{Z} \subset C$ for $j \in \mathbf{Z}$. Thus $C$ is algebraically closed, the group $G$ has order 2 and $\mathbf{Z}(j)$ is canonically isomorphic, as a $G$-module, to $\mathbf{Z}$ or to $\mathbf{Z}(1)$, depending on the parity of $j$. If $M$ is a $G$-module, we let $M(j)=M \otimes_{\mathbf{Z}} \mathbf{Z}(j)$ and $M[G]=M \otimes_{\mathbf{Z}} \mathbf{Z}[G]$; these $G$-modules fit into canonical short exact sequences

$$
0 \rightarrow M \rightarrow M[G] \rightarrow M(1) \rightarrow 0
$$

and

$$
0 \rightarrow M(1) \rightarrow M[G] \rightarrow M \rightarrow 0,
$$

which we shall refer to as the real-complex exact sequences. We denote the field of real (resp. complex) numbers by $\mathbf{R}$ (resp. $\mathbf{C}$ ). We use the term variety (over $R$ ) as a synonym for separated scheme of finite type (over $R$ ).

For the whole of \$1, we fix a variety $X$ over $R$.

1.1.1. Semi-algebraic spaces and their cohomology. The set $X(R)$ of rational points of $X$ is a locally complete (hence affine) semi-algebraic space in the sense of Delfs and Knebusch (see [DK81a, [DK81b], [DK82b], [Rob83], [DK85, Chapter I, Example 7.1], [Del91]). We shall always consider it as such; thus, a sheaf of abelian groups on $X(R)$ will refer to a sheaf on the semi-algebraic site of $X(R)$ (see [DK82b, §1, §5]). We denote by $H^{*}(X(R), \mathscr{F})$ the cohomology groups of such a sheaf. If $\mathscr{F}$ is the constant sheaf associated with $M$, we simply write $H^{*}(X(R), M)$. The semi-algebraic cohomology groups $H^{*}(X(R), M)$ are finitely generated if $M$ is a finitely generated abelian group; when $R=\mathbf{R}$, they coincide with the singular cohomology of the naive topological space $X(\mathbf{R})$, with coefficients in $M$ (see [DK82b, Proposition 6.2]). 
We also view $X(C)$ as a locally complete semi-algebraic space, namely as the space of $R$-points of the Weil restriction from $C$ to $R$ of $X_{C}=X \otimes_{R} C$. (To be precise, the Weil restriction makes sense when $X$ is quasi-projective. In general, one proceeds by choosing an affine open cover and then gluing; see [Sch94, Lemma 5.6.1].) This semi-algebraic space carries a natural action of $G$. If $\mathscr{F}$ is a $G$-equivariant sheaf of abelian groups on $X(C)$, we denote by $H_{G}^{*}(X(C), \mathscr{F})$ its equivariant cohomology groups (see $\left[\right.$ Sch94, (6.1.3)]). We simply write $H_{G}^{*}(X(C), M)$ if $\mathscr{F}$ is the constant sheaf associated with a $G$-module $M$. When $R=\mathbf{R}$, this coincides with equivariant Betti cohomology of $X(\mathbf{C})$ with coefficients in $M$. Finally, we recall that when $M$ is torsion, there are canonical isomorphisms $H^{*}(X(C), M)=H_{\text {ét }}^{*}\left(X_{C}, M\right)$ and $H_{G}^{*}(X(C), M)=H_{\text {ét }}^{*}(X, M)$ (see [Sch94, Corollary 15.3.1]). It follows, as these groups are finite when $M$ is finite and as the $G$-module $\mathbf{Q} / \mathbf{Z}(1)$ is canonically isomorphic to the group of roots of unity of $C$, that there are canonical isomorphisms $H_{G}^{*}(X(C), \mathbf{Z}(j)) \otimes_{\mathbf{Z}} \mathbf{Z}_{\ell}=H_{\text {ét }}^{*}\left(X, \mathbf{Z}_{\ell}(j)\right)$ for all primes $\ell$ and all $j$.

More generally, for any $G$-invariant locally closed semi-algebraic subset $V \subseteq X(C)$, any $G$-equivariant sheaf of abelian groups $\mathscr{F}$ on $V$, any integer $i$ and any $G$-invariant closed semi-algebraic subset $Z \subseteq V$, we denote by $H_{G, Z}^{i}(V, \mathscr{F})$ the value on $\mathscr{F}$ of the $i$ th right derived functor of the functor of $G$-invariant global sections supported on $Z$. Let $\pi: V \rightarrow V / G$ denote the quotient map (see [Bru87, Corollary 1.6]). We denote by $\mathscr{H}^{i}(G, \mathscr{F})$ the sheaf, on $V / G$, defined as the sheafification of the presheaf $U \mapsto H^{i}\left(G, \mathscr{F}\left(\pi^{-1}(U)\right)\right)$. We recall that the two spectral sequences of equivariant cohomology with support in $Z$ take the shape

$$
E_{2}^{p, q}=H_{Z / G}^{p}\left(V / G, \mathscr{H}^{q}(G, \mathscr{F})\right) \Rightarrow H_{G, Z}^{p+q}(V, \mathscr{F})
$$

and

$$
E_{2}^{p, q}=H^{p}\left(G, H_{Z}^{q}(V, \mathscr{F})\right) \Rightarrow H_{G, Z}^{p+q}(V, \mathscr{F})
$$

(see [Gro57, Théorème 5.2.1]); the latter is the Hochschild-Serre spectral sequence.

Viewing $\mathscr{F}[G]=\mathscr{F} \otimes_{\mathbf{Z}} \mathbf{Z}[G]$ as a $G$-equivariant sheaf with the diagonal action of $G$, let us now consider the spectral sequence (1.4) associated with $\mathscr{F}[G]$ (rather than with $\mathscr{F})$. As $H_{Z}^{q}(V, \mathscr{F}[G])=H_{Z}^{q}(V, \mathscr{F})[G]$, we have $E_{2}^{p, q}=0$ for $p>0$ and $E_{2}^{0, q}=H_{Z}^{q}(V, \mathscr{F})$ (see Bro94, Chapter III, Corollary 5.7, Proposition 5.9, Corollary 6.6]), hence

$$
H_{G, Z}^{i}(V, \mathscr{F}[G])=H_{Z}^{i}(V, \mathscr{F})
$$

for all $i$. Setting $\mathscr{F}(1)=\mathscr{F} \otimes_{\mathbf{Z}} \mathbf{Z}(1)$, the sequences (1.1) and (1.2) with $M=\mathbf{Z}$, tensored by $\mathscr{F}$, therefore induce long exact sequences

$$
\cdots \rightarrow H_{G, Z}^{i}(V, \mathscr{F}) \rightarrow H_{Z}^{i}(V, \mathscr{F}) \rightarrow H_{G, Z}^{i}(V, \mathscr{F}(1)) \rightarrow H_{G, Z}^{i+1}(V, \mathscr{F}) \rightarrow \cdots
$$


and

$$
\cdots \rightarrow H_{G, Z}^{i}(V, \mathscr{F}(1)) \rightarrow H_{Z}^{i}(V, \mathscr{F}) \rightarrow H_{G, Z}^{i}(V, \mathscr{F}) \rightarrow H_{G, Z}^{i+1}(V, \mathscr{F}(1)) \rightarrow \cdots
$$

for any $G$-equivariant sheaf of abelian groups $\mathscr{F}$ on $V$. We will refer to the map $H_{Z}^{i}(V, \mathscr{F}) \rightarrow H_{G, Z}^{i}(V, \mathscr{F})$ appearing in (1.7) as the norm map.

1.1.2. Notation: the class $\omega$ and its variants. For any integer $i \geq 1$, we shall denote by $\omega_{V}^{i} \in H_{G}^{i}(V, \mathbf{Z}(i))$ the image of $1 \in \mathbf{Z} / 2 \mathbf{Z}=H^{i}(G, \mathbf{Z}(i))=H_{G}^{i}(\mathrm{pt}, \mathbf{Z}(i))$ by pullback with respect to the map from $V$ to the point, and by $\omega_{V, \mathbf{Z} / 2 \mathbf{Z}}^{i} \in H_{G}^{i}(V, \mathbf{Z} / 2 \mathbf{Z})$ the image of $\omega_{V}^{i}$ by the map $H_{G}^{i}(V, \mathbf{Z}(i)) \rightarrow H_{G}^{i}(V, \mathbf{Z} / 2 \mathbf{Z})$ induced by the surjection $\mathbf{Z}(i) \rightarrow \mathbf{Z} / 2 \mathbf{Z}$. The subscript $V$ will be omitted when no confusion can arise. We shall write $\omega$ (resp., $\omega_{\mathbf{Z} / 2 \mathbf{Z}}$ ) for $\omega^{1}$ (resp., $\omega_{\mathbf{Z} / 2 \mathbf{Z}}^{1}$ ). The notation is justified by the remark that $\omega^{i}$ (resp., $\omega_{\mathbf{Z} / 2 \mathbf{Z}}^{i}$ ) coincides with the $i$-fold cup product of $\omega$ (resp., $\omega_{\mathbf{Z} / 2 \mathbf{Z}}$ ) with itself. Finally, we note that the map $H_{G, Z}^{i}(V, \mathscr{F}) \rightarrow H_{G, Z}^{i+1}(V, \mathscr{F}(1))$ which appears in (1.7) can be interpreted as the cup product with $\omega$ (see [Kah93, §A3]).

1.1.3. Cohomological dimension. For a $G$-module $M$, we shall consider the relative equivariant cohomology groups $H_{G}^{*}(X(C), X(R), M)$, defined as $H_{G}^{*}\left(X(C), j_{!} M\right)$, where $j: X(C) \backslash X(R) \hookrightarrow X(C)$ denotes the inclusion and $j_{!} M$ is the extension by zero. These groups fit into the localisation long exact sequence

$$
\cdots \longrightarrow H_{G}^{i}(X(C), X(R), M) \longrightarrow H_{G}^{i}(X(C), M) \longrightarrow H_{G}^{i}(X(R), M) \longrightarrow \cdots
$$

(induced by the exact sequence of $G$-equivariant sheaves $0 \rightarrow j_{!} M \rightarrow M \rightarrow \iota_{*} M \rightarrow 0$, where $\iota: X(R) \hookrightarrow X(C)$ denotes the inclusion). As the action of $G$ on $X(C)$ is discontinuous (see Gro57, §5.3]), it follows from the spectral sequence (1.3) that

$$
H_{G}^{i}(X(C), X(R), M)=H^{i}(X(C) / G, X(R), M)
$$

and

$$
H_{G}^{i}(X(C) \backslash X(R), M)=H^{i}((X(C) \backslash X(R)) / G, M),
$$

where $M$ now also denotes the locally constant sheaf $\mathscr{H}^{0}(G, M)$ on $(X(C) \backslash X(R)) / G$. By [Del91, Chapter II, Lemma 9.1], these two groups vanish for $i>2 \operatorname{dim}(X)$. In particular, the restriction map $H_{G}^{i}(X(C), M) \rightarrow H_{G}^{i}(X(R), M)$ is an isomorphism if $i>2 \operatorname{dim}(X)$.

1.1.4. Semi-algebraic Verdier duality and purity. Let $f: V \rightarrow W$ denote a continuous semi-algebraic map between locally complete semi-algebraic spaces over $R$. We assume that $V$ and $W$ are homology manifolds (see Del91, Chapter III, §3, Definition 1]) and denote by $\mathrm{or}_{V}$, or $_{W}$ their orientation sheaves; these are locally constant sheaves with stalks isomorphic to Z . We let or $_{V / W}=\mathscr{H}$ om $\left(f^{*}\right.$ or $_{W}$, or $\left._{V}\right)$. The main examples of such manifolds, in this article, will be the spaces $X(R), X(C)$ and $(X(C) \backslash X(R)) / G$ for a smooth variety $X$ over $R$ (loc. cit., Example 3.3). For 
any noetherian ring $\Lambda$ and any $T \in\{V, W\}$, we let $D^{+}(T, \Lambda)$ denote the derived category of bounded below complexes of sheaves of $\Lambda$-modules on the semi-algebraic site of $T$. According to [EP14, Theorem 4.1], the derived direct image functor with proper support $\mathrm{R} f_{!}: D^{+}(V, \Lambda) \rightarrow D^{+}(W, \Lambda)$, defined in [Del91, Chapter II, §8], admits a right adjoint $\mathrm{R} f^{!}: D^{+}(W, \Lambda) \rightarrow D^{+}(V, \Lambda)$.

The following statement combines Poincaré duality and a version of the Thom isomorphism in the context of semi-algebraic spaces over real closed fields.

Proposition 1.1. For any noetherian ring $\Lambda$ and any bounded complex $\mathscr{F}$ of sheaves of $\Lambda$-modules on $W$ whose cohomology sheaves are locally constant, there is a canonical isomorphism

$$
\mathrm{R} f^{!} \mathscr{F}=\left(\operatorname{or}_{V / W} \otimes_{\mathbf{Z}} f^{*} \mathscr{F}\right)[\operatorname{dim}(V)-\operatorname{dim}(W)]
$$

in $D^{+}(V, \Lambda)$.

Proof. Applying [EP14, Theorem 4.10] to $V$ and to $W$ yields a canonical isomorphism

$$
\mathrm{R} f^{!} \mathrm{or}_{W}=\operatorname{or}_{V}[\operatorname{dim}(V)-\operatorname{dim}(W)]
$$

in $D^{+}(V, \mathbf{Z})$. On the other hand, there is a canonical morphism

$$
\mathrm{R} f^{!} \mathrm{or}_{W} \otimes \mathbf{Z} f^{*} \mathscr{H} o m\left(\mathrm{or}_{W}, \mathscr{F}\right) \rightarrow \mathrm{R} f^{!} \mathscr{F}
$$

(see [KS94, Proposition 3.1.11]). It suffices to prove that the latter is an isomorphism. By the triangulated five lemma, we may assume that $\mathscr{F}$ is concentrated in degree 0 . After shrinking $W$, we may assume that $\mathscr{F}=M \otimes_{\mathbf{Z}}$ or $_{W}$ for some $\Lambda$-module $M$. The $\Lambda$-module structure is now irrelevant; we may therefore assume that $\Lambda=\mathbf{Z}$. The question being compatible with filtered direct limits, we may also assume that $M$ is a finitely generated abelian group, and then that $M=\mathbf{Z}$ or that $M=\mathbf{Z} / N \mathbf{Z}$ for some $N \geq 1$. In this case, the assertion follows from [EP14, Theorem 4.10] applied four times (to $V$ and to $W$, with coefficients $\mathbf{Z}$ and $\mathbf{Z} / N \mathbf{Z}$ ).

When $f$ is a closed embedding of pure codimension $c$, the isomorphism (1.10) with $\mathscr{F}=\Lambda=\mathbf{Z} / 2 \mathbf{Z}$ induces a canonical isomorphism

$$
H^{i-c}(V, \mathbf{Z} / 2 \mathbf{Z})=H_{V}^{i}(W, \mathbf{Z} / 2 \mathbf{Z})
$$

for any $i \in \mathbf{Z}$ (see [KS94, Proposition 3.1.12]), and hence, by forgetting the support, a Gysin map $H^{i-c}(V, \mathbf{Z} / 2 \mathbf{Z}) \rightarrow H^{i}(W, \mathbf{Z} / 2 \mathbf{Z})$. By definition, the fundamental class $s_{V / W} \in H_{V}^{c}(W, \mathbf{Z} / 2 \mathbf{Z})$ of $V$ in $W$ is the image of the constant section $1 \in H^{0}(V, \mathbf{Z} / 2 \mathbf{Z})$ by the isomorphism (1.13) for $i=c$.

More generally, if $f$ is a proper semi-algebraic map (see [Del91, Chapter II, Remark 7.6]) and if we let $c=\operatorname{dim}(W)-\operatorname{dim}(V)$, the isomorphism (1.10) yields, by 
adjunction, a canonical morphism $\mathrm{R} f_{*}\left(\operatorname{or}_{V / W} \otimes_{\mathbf{Z}} f^{*} \mathscr{F}\right) \rightarrow \mathscr{F}[c]$, since $\mathrm{R} f_{*}=\mathrm{R} f_{!}$. This morphism induces, in turn, a push-forward homomorphism

$$
f_{*}: H^{i-c}\left(V, \operatorname{or}_{V / W} \otimes_{\mathbf{Z}} f^{*} \mathscr{F}\right) \rightarrow H^{i}(W, \mathscr{F})
$$

for any $i \in \mathbf{Z}$. Pull-back and proper push-forward are related by the formula

$$
f_{*}\left(\alpha \smile f^{*} \beta\right)=f_{*} \alpha \smile \beta,
$$

valid in $H^{i+j+c}\left(W, \mathscr{F} \otimes_{\mathbf{Z}}^{\mathbf{L}} \mathscr{G}\right)$ for all $\alpha \in H^{i}\left(V\right.$, or $\left._{V / W} \otimes_{\mathbf{Z}} f^{*} \mathscr{F}\right), \beta \in H^{j}(W, \mathscr{G})$, all $i, j$, and all bounded complexes of sheaves of abelian groups $\mathscr{F}$ and $\mathscr{G}$ on $W$ with locally constant cohomology sheaves (and therefore also in $H^{i+j+c}\left(W, \mathscr{F} \otimes_{\mathbf{z}} \mathscr{G}\right)$ if $\mathscr{F}$ and $\mathscr{G}$ are themselves just locally constant sheaves). To prove (1.15), we note that the map $\beta \mapsto f_{*}\left(\alpha \smile f^{*} \beta\right)$ is induced by a morphism $\mathscr{G} \rightarrow \mathscr{F} \otimes_{\mathbf{Z}}^{\mathbf{L}} \mathscr{G}[i+c]$ in $D^{+}(W, \mathbf{Z})$ that depends functorially on $\mathscr{G}$. In view of the general fact stated below (which could also be taken as a definition for the cup product), this map can be interpreted, for all $j$ and all $\mathscr{G}$, as the cup product with the class in $H^{i+c}(W, \mathscr{F})$ obtained by taking $j=0, \mathscr{G}=\mathbf{Z}, \beta=1$, i.e., with the class $f_{*} \alpha$.

Fact 1.2. Let $\mathscr{A}_{1}, \mathscr{A}_{2}$ be bounded above complexes of sheaves of abelian groups on $W$ and let $x_{1} \in H^{0}\left(W, \mathscr{A}_{1}\right), x_{2} \in H^{0}\left(W, \mathscr{A}_{2}\right)$. We set $\mathscr{A}_{3}=\mathscr{A}_{1} \otimes_{\mathbf{Z}}^{\mathbf{L}} \mathscr{A}_{2}$ and $x_{3}=x_{1} \smile x_{2} \in H^{0}\left(W, \mathscr{A}_{3}\right)$. Letting $\varphi_{i}: \mathbf{Z} \rightarrow \mathscr{A}_{i}$ denote the morphism in $D^{-}(W, \mathbf{Z})$ corresponding to $x_{i}$, we have $\varphi_{3}=\left(\operatorname{Id}_{\mathscr{A}_{1}} \otimes \varphi_{2}\right) \circ \varphi_{1}$.

As explained in [Del73, §3.2.6], when $V$ has pure dimension $d$ and $W$ is a point, the isomorphism (1.10) with $\mathscr{F}=\Lambda=\mathbf{Z} / N \mathbf{Z}$ for a divisible enough $N \geq 1$ induces, for any locally constant sheaf $\mathscr{G}$ of abelian groups of finite exponent on $V$, a perfect Poincaré duality pairing

$$
H_{c}^{i}(V, \mathscr{G}) \times H^{d-i}\left(V, \mathscr{G}^{\vee} \otimes_{\mathbf{Z}} \text { or }_{V}\right) \rightarrow \mathbf{Q} / \mathbf{Z}
$$

for every $i \in \mathbf{Z}$, where $H_{c}^{i}(V, \mathscr{G})$ denotes semi-algebraic cohomology with complete supports (see [Del91, Chapter II, §1]) and $\mathscr{G}^{`}=\mathscr{H} o m(\mathscr{G}, \mathbf{Q} / \mathbf{Z})$.

1.1.5. Equivariant purity. We keep the notation of $\$ 1.1 .4$ and assume, in addition, that the group $G=\operatorname{Gal}(C / R)$ acts on $V$ and $W$ and that $f$ is $G$-equivariant; thus, the sheaves or $_{V}$, or $_{W}$, and or $_{V / W}$ are $G$-equivariant. Using a finite-dimensional approximation of the Borel construction, we now transfer the results of \$1.1.4 to the setting of equivariant cohomology. Let $G$ act on the $n$-dimensional semi-algebraic unit sphere $S^{n} \subset R^{n+1}$ by the antipodal involution. For $T \in\{V, W\}$, the diagonal action of $G$ on $T \times S^{n}$ is free and discontinuous, hence the quotient semi-algebraic space $\left(T \times S^{n}\right) / G$ is again a homology manifold. Moreover, if $p: T \times S^{n} \rightarrow T$ denotes the first projection, then for any $G$-equivariant sheaf $\mathscr{F}$ of abelian groups on $T$, the 
$G$-equivariant sheaf $p^{*} \mathscr{F}$ uniquely descends to a sheaf $\mathscr{F}^{\prime}$ on $\left(T \times S^{n}\right) / G$ and for any $G$-invariant closed semi-algebraic subset $Z \subseteq T$, there are canonical isomorphisms

$$
H_{G, Z}^{i}(T, \mathscr{F})=H_{G, Z \times S^{n}}^{i}\left(T \times S^{n}, p^{*} \mathscr{F}\right)=H_{\left(Z \times S^{n}\right) / G}^{i}\left(\left(T \times S^{n}\right) / G, \mathscr{F}^{\prime}\right)
$$

for every $i<n$, as follows from (1.3) and from the Leray spectral sequence for $p$. If $\mathscr{F}$ is a locally constant $G$-equivariant sheaf of abelian groups (by which we mean that it is a $G$-equivariant sheaf of abelian groups which, as a sheaf of abelian groups, is locally constant), then $\mathscr{F}^{\prime}$ is locally constant. We note, however, that $\mathscr{F}$ being a constant sheaf does not imply that $\mathscr{F}^{\prime}$ is constant.

As a consequence, when $f$ is a closed embedding of pure codimension $c$, applying Proposition 1.1 to the inclusion of $\left(V \times S^{n}\right) / G$ in $\left(W \times S^{n}\right) / G$ for a large enough $n$ yields a canonical isomorphism

$$
H_{G}^{i-c}\left(V, \operatorname{or}_{V / W} \otimes_{\mathbf{z}} f^{*} \mathscr{F}\right)=H_{G, V}^{i}(W, \mathscr{F})
$$

for any $i \in \mathbf{Z}$ and any locally constant $G$-equivariant sheaf $\mathscr{F}$ of abelian groups on $W$. Similarly, when $f$ is a proper semi-algebraic map, considering (1.14) for the map $\left(V \times S^{n}\right) / G \rightarrow\left(W \times S^{n}\right) / G$ induced by $f$ for a large enough $n$ leads to a push-forward homomorphism

$$
f_{*}: H_{G}^{i-c}\left(V, \mathrm{or}_{V / W} \otimes_{\mathbf{z}} f^{*} \mathscr{F}\right) \rightarrow H_{G}^{i}(W, \mathscr{F})
$$

for any $i \in \mathbf{Z}$ and any locally constant $G$-equivariant sheaf $\mathscr{F}$ of abelian groups on $W$, where $c=\operatorname{dim}(W)-\operatorname{dim}(V)$.

In particular, if $X$ is a smooth variety over $R$, purely of dimension $d$, we obtain a canonical isomorphism

$$
H_{G}^{i-d}(X(R), \mathbf{Z} / 2 \mathbf{Z})=H_{G, X(R)}^{i}(X(C), \mathbf{Z} / 2 \mathbf{Z})
$$

for any $i \in \mathbf{Z}$ by taking $\mathscr{F}=\Lambda=\mathbf{Z} / 2 \mathbf{Z}$ and $V=X(R), W=X(C)$. The equivariant fundamental class $s_{G, X(R) / X(C)} \in H_{G, X(R)}^{d}(X(C), \mathbf{Z} / 2 \mathbf{Z})$ of $X(R)$ in $X(C)$ is the image of the constant section $1 \in H_{G}^{0}(X(R), \mathbf{Z} / 2 \mathbf{Z})$ by the isomorphism (1.20) for $i=d$.

We note that for any smooth variety $X$ of pure dimension $d$ over $R$, there is a canonical isomorphism of $G$-equivariant sheaves or ${ }_{X(C)}=\mathbf{Z}(d)$ (see Del91, Chapter IV, $\S 1$, Example 1.7]). As a consequence, for any smooth variety $X$ over $R$, any smooth subvariety $Y \subseteq X$ of pure codimension $k$ and any $G$-module $M$, we find that $\operatorname{or}_{Y(C) / X(C)}=\mathbf{Z}(-k)$ and we obtain a canonical isomorphism

$$
H_{G}^{i-2 k}(Y(C), M(-k))=H_{G, Y(C)}^{i}(X(C), M)
$$

for any $i \in \mathbf{Z}$, by taking $\Lambda=\mathbf{Z}, \mathscr{F}=M, V=Y(C), W=X(C)$ in (1.18); and hence, by forgetting the support, also a Gysin map $H_{G}^{i-2 k}(Y(C), M(-k)) \rightarrow H_{G}^{i}(X(C), M)$.

More generally, for any proper morphism $f: Y \rightarrow X$ of smooth equidimensional varieties over $R$ and for any $G$-module $M$, if we let $k=\operatorname{dim}(X)-\operatorname{dim}(Y)$, we obtain, 
in view of (1.19), a push-forward homomorphism

$$
f_{*}: H_{G}^{i-2 k}(Y(C), M(-k)) \rightarrow H_{G}^{i}(X(C), M)
$$

for any $i \in \mathbf{Z}$, since or $_{Y(C) / X(C)}=\mathbf{Z}(-k)$. The projection formula

$$
f_{*}\left(\alpha \smile f^{*} \beta\right)=f_{*} \alpha \smile \beta
$$

now holds in $H_{G}^{i+j+2 k}\left(X(C),\left(M \otimes_{\mathbf{Z}} M^{\prime}\right)(k)\right)$ for all $G$-modules $M$ and $M^{\prime}$, all $\alpha \in H_{G}^{i}(Y(C), M), \beta \in H_{G}^{j}\left(X(C), M^{\prime}\right)$, and all $i, j$ (see (1.15)).

1.2. Canonical decompositions. Let $\mathscr{F}$ be a locally constant sheaf of abelian groups on $X(R)$, with stalks isomorphic to $\mathbf{Z}$, viewed as a $G$-equivariant sheaf with the trivial action. For any $G$-module $M$ and any $i \geq 0$, as $G$ acts trivially on $X(R)$, there is a canonical isomorphism

$$
H_{G}^{i}\left(X(R), \mathscr{F} \otimes_{\mathbf{Z}} M\right)=H^{i}\left(X(R), \mathscr{F} \otimes_{\mathbf{Z}}^{\mathbf{L}} \mathrm{R} \Gamma(G, M)\right) .
$$

Indeed, for any $G$-equivariant sheaf of abelian groups $\mathscr{G}$ on $X(R)$, there is a canonical isomorphism $H_{G}^{i}(X(R), \mathscr{G})=H^{i}\left(X(R), \mathrm{R} \mathscr{H}^{0}(G, \mathscr{G})\right)$, where $\mathrm{R} \mathscr{H}^{0}(G,-)$ denotes the total right derived functor of the functor $\mathscr{H}^{0}(G,-)$ introduced just before (1.3); as $\operatorname{R} \mathscr{H}^{0}\left(G, \mathscr{F} \otimes_{\mathbf{Z}} M\right)=\mathscr{F} \otimes_{\mathbf{Z}}^{\mathbf{L}} \mathrm{R} \Gamma(G, M)$, this yields (1.24).

1.2.1. With $\mathbf{Z} / 2 \mathbf{Z}$ coefficients. Let us apply (1.24) to $M=\mathbf{Z} / 2 \mathbf{Z}$. As $\mathbf{Z} / 2 \mathbf{Z}$ is a field, there exists, in the derived category of $\mathbf{Z} / 2 \mathbf{Z}$-modules, a canonical isomorphism

$$
\mathrm{R} \Gamma(G, \mathbf{Z} / 2 \mathbf{Z})=\bigoplus_{q \geq 0} H^{q}(G, \mathbf{Z} / 2 \mathbf{Z})[-q]
$$

(There is, in fact, a unique isomorphism inducing the identity on the cohomology groups.) As $H^{q}(G, \mathbf{Z} / 2 \mathbf{Z})=\mathbf{Z} / 2 \mathbf{Z}$ for all $q \geq 0$, a canonical decomposition

$$
H_{G}^{i}(X(R), \mathbf{Z} / 2 \mathbf{Z})=\bigoplus_{0 \leq p \leq i} H^{p}(X(R), \mathbf{Z} / 2 \mathbf{Z})
$$

results, for any $i$. We note that as the category of $\mathbf{Z} / 2 \mathbf{Z}$-modules is semisimple, the derived cup product map $\mathrm{R} \Gamma(G, \mathbf{Z} / 2 \mathbf{Z}) \otimes_{\mathbf{Z} / 2 \mathbf{Z}}^{\mathbf{L}} \mathrm{R}(G, \mathbf{Z} / 2 \mathbf{Z}) \rightarrow \mathrm{R} \Gamma(G, \mathbf{Z} / 2 \mathbf{Z})$ has to coincide, via (1.25), with the direct sum, over all $r$, of the cup product maps

$$
\bigoplus_{p+q=r} H^{p}(G, \mathbf{Z} / 2 \mathbf{Z}) \otimes_{\mathbf{Z} / 2 \mathbf{Z}} H^{q}(G, \mathbf{Z} / 2 \mathbf{Z}) \rightarrow H^{r}(G, \mathbf{Z} / 2 \mathbf{Z})
$$

shifted by $-r$. The cup product of $x \in H_{G}^{k}(X(R), \mathbf{Z} / 2 \mathbf{Z}), y \in H_{G}^{\ell}(X(R), \mathbf{Z} / 2 \mathbf{Z})$ in $H_{G}^{k+\ell}(X(R), \mathbf{Z} / 2 \mathbf{Z})$ can therefore be written, in terms of (1.26), as

$$
x \smile y=\left(\sum_{p+q=r} x_{p} \smile y_{q}\right)_{0 \leq r \leq k+\ell}
$$

if $x=\left(x_{p}\right)_{0 \leq p \leq k}$ and $y=\left(y_{q}\right)_{0 \leq q \leq \ell}$. 
As an alternative way to obtain (1.26), one can identify $H_{G}^{i}(X(R), \mathbf{Z} / 2 \mathbf{Z})$ with $H^{i}\left(X(R) \times\left(S^{n} / G\right), \mathbf{Z} / 2 \mathbf{Z}\right)$ for some $n>i$, via the Borel construction (see \$1.1.5), and then apply the Künneth formula. This leads to the same decomposition, as we have $\tau_{\leq i} \mathrm{R} \Gamma(G, \mathbf{Z} / 2 \mathbf{Z})=\tau_{\leq i} \mathrm{R} \pi_{*} \mathbf{Z} / 2 \mathbf{Z}$ in $D^{+}(X(R), \mathbf{Z} / 2 \mathbf{Z})$ if $\pi: X(R) \times\left(S^{n} / G\right) \rightarrow X(R)$ denotes the first projection.

1.2.2. With integral coefficients. Let us now apply (1.24) to $M=\mathbf{Z}(j)$ for $j \in \mathbf{Z}$. In the derived category of abelian groups, there exists a canonical isomorphism

$$
\mathrm{R} \Gamma(G, \mathbf{Z}(j))=\bigoplus_{q \geq 0} H^{q}(G, \mathbf{Z}(j))[-q]
$$

as can be seen from the standard explicit complex representing the total cohomology of the cyclic group $G$. (There is, in fact, a unique isomorphism inducing the identity on the cohomology groups.) Letting $\mathscr{F}(j)=\mathscr{F} \otimes_{\mathbf{Z}} \mathbf{Z}(j)$, a canonical decomposition

$$
H_{G}^{i}(X(R), \mathscr{F}(j))=\bigoplus_{p+q=i} H^{p}\left(X(R), \mathscr{F} \otimes_{\mathbf{Z}} H^{q}(G, \mathbf{Z}(j))\right)
$$

results, for any $i$. We note that $H^{q}(G, \mathbf{Z}(j))=0$ if $q \not \equiv j \bmod 2, H^{q}(G, \mathbf{Z}(j))=\mathbf{Z} / 2 \mathbf{Z}$ if $q \equiv j \bmod 2$ and $q>0$, and $H^{q}(G, \mathbf{Z}(j))=\mathbf{Z}$ if $q=0$ and $j$ is even. By mapping $H^{i}(X(R), \mathscr{F})$ to $H^{i}(X(R), \mathscr{F} / 2 \mathscr{F})=H^{i}(X(R), \mathbf{Z} / 2 \mathbf{Z})$ in the obvious way, we thus obtain a natural map

$$
H_{G}^{i}(X(R), \mathscr{F}(j)) \rightarrow \underset{\substack{0 \leq p \leq i \\ p \equiv i-j \bmod 2}}{\bigoplus} H^{p}(X(R), \mathbf{Z} / 2 \mathbf{Z}),
$$

which is an isomorphism if $i>\operatorname{dim}(X)$ (see $[\mathrm{DK} 82 \mathrm{~b}, \S 5]$ ) or if $j$ is odd.

If $Y \subset X$ is a closed subvariety of $X$, the exact same reasoning yields a canonical decomposition

$$
H_{G, Y(R)}^{i}(X(R), \mathscr{F}(j))=\bigoplus_{p+q=i} H_{Y(R)}^{p}\left(X(R), \mathscr{F} \otimes_{\mathbf{Z}} H^{q}(G, \mathbf{Z}(j))\right) .
$$

We warn the reader that the formula (1.28) does not describe cup products in equivariant cohomology with integral coefficients in terms of the decompositions (1.30).

1.2.3. Change of coefficients. Krasnov [Kra94, Theorem 1.2, Theorem 1.3] has shown that the above canonical decompositions of cohomology with coefficients in $\mathscr{F}(j)$ and in $\mathbf{Z} / 2 \mathbf{Z}$ are compatible in the following sense: for any $i$ and $j$, the reduction map

$$
H_{G}^{i}(X(R), \mathscr{F}(j)) \rightarrow H_{G}^{i}\left(X(R), \mathscr{F}(j) \otimes_{\mathbf{Z}} \mathbf{Z} / 2 \mathbf{Z}\right)=H_{G}^{i}(X(R), \mathbf{Z} / 2 \mathbf{Z}),
$$


the natural map (1.31) and the decomposition (1.26) fit into a commutative square

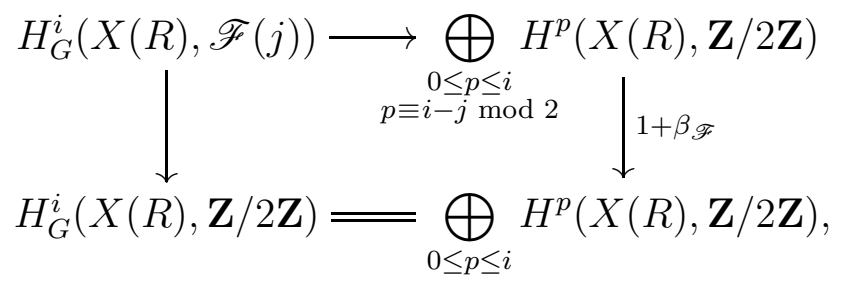

where the right-hand vertical arrow is the sum of the twisted Bockstein homomorphisms $\beta_{\mathscr{F}}: H^{p}(X(R), \mathbf{Z} / 2 \mathbf{Z}) \rightarrow H^{p+1}(X(R), \mathbf{Z} / 2 \mathbf{Z})$, defined as the boundary maps of the short exact sequence $0 \rightarrow \mathbf{Z} / 2 \mathbf{Z} \rightarrow \mathscr{F} \otimes_{\mathbf{Z}} \mathbf{Z} / 4 \mathbf{Z} \rightarrow \mathbf{Z} / 2 \mathbf{Z} \rightarrow 0$, for $0 \leq p \leq i-1$, and of the identity maps $H^{p}(X(R), \mathbf{Z} / 2 \mathbf{Z}) \rightarrow H^{p}(X(R), \mathbf{Z} / 2 \mathbf{Z})$, for $0 \leq p \leq i$.

1.2.4. Effect on the real-complex short exact sequence. Let us consider the effect of the decomposition (1.26) on the real-complex exact sequence (1.1) for $M=\mathbf{Z} / 2 \mathbf{Z}$. Applying the functor $\operatorname{R} \Gamma(G,-)$ to this short exact sequence yields, via the canonical isomorphisms (1.25) and $\mathrm{R} \Gamma(G, \mathbf{Z} / 2 \mathbf{Z}[G])=\mathbf{Z} / 2 \mathbf{Z}$, a distinguished triangle

$$
\bigoplus_{q \geq 0} \mathbf{Z} / 2 \mathbf{Z}[-q] \rightarrow \mathbf{Z} / 2 \mathbf{Z} \rightarrow \bigoplus_{q \geq 0} \mathbf{Z} / 2 \mathbf{Z}[-q] \rightarrow \bigoplus_{q \geq-1} \mathbf{Z} / 2 \mathbf{Z}[-q]
$$

The first map of (1.34) is the natural projection, the second map vanishes, and the third is the natural inclusion. (Indeed, these induce the correct maps after passing to cohomology, and the category of $\mathbf{Z} / 2 \mathbf{Z}$-modules is semisimple.) Thus, the decomposition (1.26) identifies the canonical map $H_{G}^{n}(X(R), \mathbf{Z} / 2 \mathbf{Z}) \rightarrow H^{n}(X(R), \mathbf{Z} / 2 \mathbf{Z})$ with the projection map $H^{0}(X(R), \mathbf{Z} / 2 \mathbf{Z}) \oplus \cdots \oplus H^{n}(X(R), \mathbf{Z} / 2 \mathbf{Z}) \rightarrow H^{n}(X(R), \mathbf{Z} / 2 \mathbf{Z})$ and it identifies the map $H_{G}^{n}(X(R), \mathbf{Z} / 2 \mathbf{Z}) \rightarrow H_{G}^{n+1}(X(R), \mathbf{Z} / 2 \mathbf{Z})$ induced by (1.1) with the inclusion $\bigoplus_{0 \leq p \leq n} H^{p}(X(R), \mathbf{Z} / 2 \mathbf{Z}) \subseteq \bigoplus_{0 \leq p \leq n+1} H^{p}(X(R), \mathbf{Z} / 2 \mathbf{Z})$.

1.2.5. Decomposition of $\omega$. The class $\omega_{\mathbf{Z} / 2 \mathbf{Z}}^{i} \in H_{G}^{i}(X(R), \mathbf{Z} / 2 \mathbf{Z})$ (defined in \$1.1.2) is mapped, by the canonical decomposition (11.26), to the unit element of the graded ring $\bigoplus_{0 \leq p \leq i} H^{p}(X(R), \mathbf{Z} / 2 \mathbf{Z})$, since this is so when $X$ is a point and since (1.26) is compatible with pull-backs.

1.2.6. Covariant functoriality. Let $X$ and $Y$ be smooth equidimensional varieties over $R$. Let $c=\operatorname{dim}(X)-\operatorname{dim}(Y)$. Let $f: Y \rightarrow X$ be a proper morphism. The push-forward map $f_{*}: H_{G}^{i-c}(Y(R), \mathbf{Z} / 2 \mathbf{Z}) \rightarrow H_{G}^{i}(X(R), \mathbf{Z} / 2 \mathbf{Z})$ constructed in (1.19) can be regarded, via the decompositions (1.26), as a map

$$
f_{*}: \bigoplus_{0 \leq p \leq i-c} H^{p}(Y(R), \mathbf{Z} / 2 \mathbf{Z}) \rightarrow \bigoplus_{0 \leq p \leq i} H^{p}(X(R), \mathbf{Z} / 2 \mathbf{Z})
$$

It follows at once from the interpretation of (1.26) in terms of the Borel construction and of the Künneth formula (see \$1.2.1), and from the projection formula (see (1.15) ), 
that (1.35) coincides with the direct sum, over $p \in\{0, \ldots, i-c\}$, of the push-forward $\operatorname{map} f_{*}: H^{p}(X(R), \mathbf{Z} / 2 \mathbf{Z}) \rightarrow H^{p+c}(X(R), \mathbf{Z} / 2 \mathbf{Z})$ constructed in (1.14).

1.3. On the normal bundle of $X(R)$ in $X(C)$. We fix a smooth variety $X$ over $R$. Making use of the invertibility of the equivariant Euler class of the normal bundle of $X(R)$ in $X(C)$, viewed as an element of the non-equivariant cohomology ring of $X(R)$ via the canonical decompositions of $\$ 1.2$, we investigate, in this section, the long exact sequence of equivariant cohomology of $X(C)$ with support in $X(R)$.

1.3.1. With $\mathbf{Z} / 2 \mathbf{Z}$ coefficients. With $\mathbf{Z} / 2 \mathbf{Z}$ coefficients, we show that this long exact sequence decomposes into canonically split short exact sequences.

Proposition 1.3. Let $X$ be a smooth variety over $R$. For every $i \geq 0$, the sequence

$$
0 \rightarrow H_{G, X(R)}^{i}(X(C), \mathbf{Z} / 2 \mathbf{Z}) \rightarrow H_{G}^{i}(X(C), \mathbf{Z} / 2 \mathbf{Z}) \rightarrow H_{G}^{i}(X(C) \backslash X(R), \mathbf{Z} / 2 \mathbf{Z}) \rightarrow 0
$$

is exact and canonically split.

The exactness of this sequence and the role of the equivariant Euler class appear in [AP93, Proposition 5.3.7] when $i \gg 0$ (in the setting of Poincaré duality spaces) and in vH00b, Lemma 2.6] for all $i \geq 0$. The description of a canonical splitting is new and will be of importance in the sequel. The remainder of $\$ 1.3 .1$ is devoted to constructing a canonical retraction of the left arrow of the above sequence, for any $i$, thus proving Proposition 1.3. From now on, we assume, as we may, that $X$ is irreducible, and we let $d=\operatorname{dim}(X)$.

Definition 1.4. For the sake of simplicity, let us write $H^{p}=H^{p}(X(R), \mathbf{Z} / 2 \mathbf{Z})$. We denote by $\gamma \in H^{0} \oplus \cdots \oplus H^{d}$ the image of the equivariant fundamental class of $X(R)$ in $X(C)$, introduced in $\$ 1.1 .5$, by the composition

$$
H_{G, X(R)}^{d}(X(C), \mathbf{Z} / 2 \mathbf{Z}) \rightarrow H_{G}^{d}(X(C), \mathbf{Z} / 2 \mathbf{Z}) \rightarrow H_{G}^{d}(X(R), \mathbf{Z} / 2 \mathbf{Z})=H^{0} \oplus \cdots \oplus H^{d}
$$

of the forgetful map, the restriction map, and the canonical decomposition (1.26).

Lemma 1.5. The class $\gamma$ is invertible in the graded ring $H^{0} \oplus \cdots \oplus H^{d}$. In other words, its degree 0 component is the constant section $1 \in H^{0}$.

Proof. Let us denote the equivariant fundamental class by $s \in H_{G, X(R)}^{d}(X(C), \mathbf{Z} / 2 \mathbf{Z})$. For any $i$, the composition of the equivariant purity isomorphism

$$
H_{G}^{i-d}(X(R), \mathbf{Z} / 2 \mathbf{Z}) \stackrel{\sim}{\longrightarrow} H_{G, X(R)}^{i}(X(C), \mathbf{Z} / 2 \mathbf{Z})
$$

(see (1.20) ) with the forgetful map

$$
f_{i}: H_{G, X(R)}^{i}(X(C), \mathbf{Z} / 2 \mathbf{Z}) \rightarrow H_{G}^{i}(X(C), \mathbf{Z} / 2 \mathbf{Z})
$$

and the restriction map

$$
g_{i}: H_{G}^{i}(X(C), \mathbf{Z} / 2 \mathbf{Z}) \rightarrow H_{G}^{i}(X(R), \mathbf{Z} / 2 \mathbf{Z})
$$


sends $x \in H_{G}^{i-d}(X(R), \mathbf{Z} / 2 \mathbf{Z})$ to $x \smile g_{d}\left(f_{d}(s)\right) \in H_{G}^{i}(X(R), \mathbf{Z} / 2 \mathbf{Z})$. (Indeed, the two maps (1.36) and $g_{i} \circ f_{i}$ are induced by maps in the derived category of sheaves on $\left(X(R) \times S^{n}\right) / G$ that do not depend on $i$. Hence so is their composition, which can therefore be interpreted, for all $i$, as the cup product with a fixed class in $H_{G}^{d}(X(R), \mathbf{Z} / 2 \mathbf{Z}$ ) (see Fact 1.2). To compute this class, one simply takes $i=d$.) By $\$ 1.2 .1$, this map can therefore be identified, via the canonical decompositions (1.26), with the map

$$
H^{0} \oplus \cdots \oplus H^{i-d} \rightarrow H^{0} \oplus \cdots \oplus H^{i}
$$

given by multiplication by $\gamma$ (see (1.28)). On the other hand, as the groups $H_{G}^{i}(X(C) \backslash X(R), \mathbf{Z} / 2 \mathbf{Z})$ and $H_{G}^{i}(X(C), X(R), \mathbf{Z} / 2 \mathbf{Z}$ ) vanish for $i \gg 0$ (see \$1.1.3), the maps $f_{i}$ and $g_{i}$ are isomorphisms for $i \gg 0$. Hence $\gamma$ is invertible (note that $H^{0} \oplus \cdots \oplus H^{i-d}=H^{0} \oplus \cdots \oplus H^{d}$ for $i \gg 0$, by [Del91, Chapter II, Lemma 9.1]).

Thanks to Lemma 1.5, we may now consider, for any $i$, the composition

$$
H_{G}^{i}(X(C), \mathbf{Z} / 2 \mathbf{Z}) \rightarrow H_{G, X(R)}^{i}(X(C), \mathbf{Z} / 2 \mathbf{Z})
$$

of the following maps: first the restriction map $g_{i}$, then the canonical decomposition $H_{G}^{i}(X(R), \mathbf{Z} / 2 \mathbf{Z})=H^{0} \oplus \cdots \oplus H^{i}$, then the map $H^{0} \oplus \cdots \oplus H^{i} \rightarrow H^{0} \oplus \cdots \oplus H^{i-d}$ given by multiplication by $\gamma^{-1}$ followed by projection onto the first $i-d+1$ summands, then the canonical decomposition $H^{0} \oplus \cdots \oplus H^{i-d}=H_{G}^{i-d}(X(R), \mathbf{Z} / 2 \mathbf{Z})$ and finally the isomorphism (1.36). According to the proof of Lemma 1.5, the map (1.40) is indeed a retraction of $f_{i}$. Thus, Proposition 1.3 is established.

Remarks 1.6. (i) If $R=\mathbf{R}$, Krasnov has checked that $\gamma$ coincides with the total Stiefel-Whitney class of the tangent bundle of $X(R)$ (see [Kra94, Theorem 2.1]).

(ii) Let $\pi_{0}(X(R))$ denote the set of semi-algebraic connected components of $X(R)$. For $V \in \pi_{0}(X(R))$, let $s_{V}$ denote the image, in $H_{\mathrm{ett}}^{d}(X, \mathbf{Z} / 2 \mathbf{Z})=H_{G}^{d}(X(C), \mathbf{Z} / 2 \mathbf{Z})$, of the equivariant fundamental class of $V$ in $X(C)$. For $x \in X(R)$, the image of $s_{V}$ by the evaluation map ev ${ }_{x}: H_{\text {ett }}^{d}(X, \mathbf{Z} / 2 \mathbf{Z}) \rightarrow H_{\text {êt }}^{d}(x, \mathbf{Z} / 2 \mathbf{Z})=\mathbf{Z} / 2 \mathbf{Z}$ coincides with the evaluation at $x$ of the image, by the map (1.39) for $i=d$, of the non-zero element of $H^{0}$ supported on $V$. In view of Lemma [1.5, it follows that $\mathrm{ev}_{x}\left(s_{V}\right)=0$ if and only if $x \notin V$ : the $s_{V}$ 's form a canonical family of elements of $H_{\text {êt }}^{d}(X, \mathbf{Z} / 2 \mathbf{Z})$ which separate the semi-algebraic connected components of $X(R)$. This gives a further (and entirely canonical) answer to a question of Colliot-Thélène and Parimala [CTP90, Remark 2.4.4]. At least for $R=\mathbf{R}$, answers to this question had already been given by Krasnov and by van Hamel, see [vH00b, §2.3].

(iii) We have constructed not only a retraction of $f_{i}$ but also one of $g_{i} \circ f_{i}$ since the map (1.40) factors, by definition, through $g_{i}$. The kernel of this retraction of $g_{i} \circ f_{i}$, and therefore also the cokernel of $g_{i} \circ f_{i}$, is canonically isomorphic to $H^{i-d+1} \oplus \cdots \oplus H^{i}$. 
1.3.2. With integral coefficients. The assertion obtained by replacing $\mathbf{Z} / 2 \mathbf{Z}$ with $\mathbf{Z}(j)$ in the statement of Proposition 1.3 fails: already when $X=\mathbf{A}_{R}^{1}$ and $i=j=1$, the group $H_{G, X(R)}^{i}(X(C), \mathbf{Z}(j))$ is infinite while $H_{G}^{i}(X(C), \mathbf{Z}(j))$ has order 2 . It does hold, however, when $j$ has the correct parity, as we now show.

Proposition 1.7. Let $X$ be a smooth, irreducible variety over $R$, of dimension $d$. Let $j \equiv d-1 \bmod 2$. For every $i \geq 0$, the sequence

$$
0 \rightarrow H_{G, X(R)}^{i}(X(C), \mathbf{Z}(j)) \rightarrow H_{G}^{i}(X(C), \mathbf{Z}(j)) \rightarrow H_{G}^{i}(X(C) \backslash X(R), \mathbf{Z}(j)) \rightarrow 0
$$

is exact and canonically split.

As in \$1.3.1, we shall prove Proposition 1.7 by describing a canonical retraction of the left arrow of the above sequence. Let us denote $\widetilde{\mathbf{Z}}=$ or $_{X(R)}$ and $\widetilde{\mathbf{Z}}(j)=\widetilde{\mathbf{Z}} \otimes_{\mathbf{Z}} \mathbf{Z}(j)$.

Proof. In view of the canonical isomorphism of $G$-equivariant sheaves or ${ }_{X(C)}=\mathbf{Z}(d)$, putting together the equivariant purity isomorphisms (1.18) and the square (1.33) yields a commutative diagram

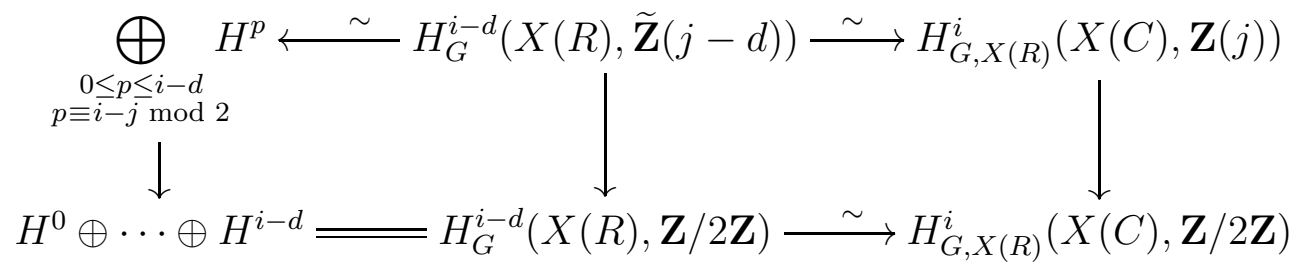

in which the leftmost vertical map is $1+\beta_{\widetilde{\mathbf{Z}}}$ (see \$1.2.3) and in which the top left horizontal arrow is an isomorphism because $j-d$ is odd (see \$1.2.2). The map $1+\beta_{\widetilde{\mathbf{z}}}$ admits an obvious canonical retraction, namely the projection map. Following the isomorphisms in the above diagram, this yields a canonical retraction of the rightmost vertical map. Composing it with the map (1.40) constructed in \$1.3.1 and with the natural map $H_{G}^{i}(X(C), \mathbf{Z}(j)) \rightarrow H_{G}^{i}(X(C), \mathbf{Z} / 2 \mathbf{Z})$, we obtain the desired retraction.

Refining these ideas leads to the following integral variant of [vH00b, Theorem 2.8] and [Kra94, Corollary 3.2]. We shall use it in \$5] and in [BW18].

Proposition 1.8. Let $X$ be a smooth, irreducible variety over $R$, of dimension $d$. For any $i \geq 0$ and any $j \in \mathbf{Z}$, the composition

$$
H_{G}^{i}(X(C), \mathbf{Z}(j)) \rightarrow \underset{\substack{0 \leq p<i-d \\ p \equiv i-j \bmod 2}}{\bigoplus} H^{p}(X(R), \mathbf{Z} / 2 \mathbf{Z})
$$

of the restriction map $H_{G}^{i}(X(C), \mathbf{Z}(j)) \rightarrow H_{G}^{i}(X(R), \mathbf{Z}(j))$, of the map (1.31), and of the natural projection is surjective. 
Proof. We shall in fact prove the surjectivity (and even produce a canonical section) of the map obtained by composing (1.41) with the forgetful map

$$
H_{G, X(R)}^{i}(X(C), \mathbf{Z}(j)) \rightarrow H_{G}^{i}(X(C), \mathbf{Z}(j))
$$

and with the equivariant purity isomorphism

$$
H_{G}^{i-d}(X(R), \widetilde{\mathbf{Z}}(j-d)) \stackrel{\sim}{\longrightarrow} H_{G, X(R)}^{i}(X(C), \mathbf{Z}(j))
$$

(see (1.18) ). As in the proof of Lemma 1.5, we remark that the composition of the maps (1.43) and (1.42) with the restriction map $H_{G}^{i}(X(C), \mathbf{Z}(j)) \rightarrow H_{G}^{i}(X(R), \mathbf{Z}(j))$ exists at the derived level: this map can be written as $H^{i-d}(X(R), e)$ for some map

$$
e: \widetilde{\mathbf{Z}} \otimes_{\mathbf{Z}}^{\mathbf{L}} \mathrm{R} \Gamma(G, \mathbf{Z}(j-d)) \rightarrow \mathrm{R} \Gamma(G, \mathbf{Z}(j))[d]
$$

in the derived category of sheaves of abelian groups on $X(R)$. In view of the decomposition (1.29), in view of \$1.2.3, and in view of the proof of Lemma 1.5, this map fits into the following commutative diagram, in which $\tau_{>0}$ denotes the truncation functor:

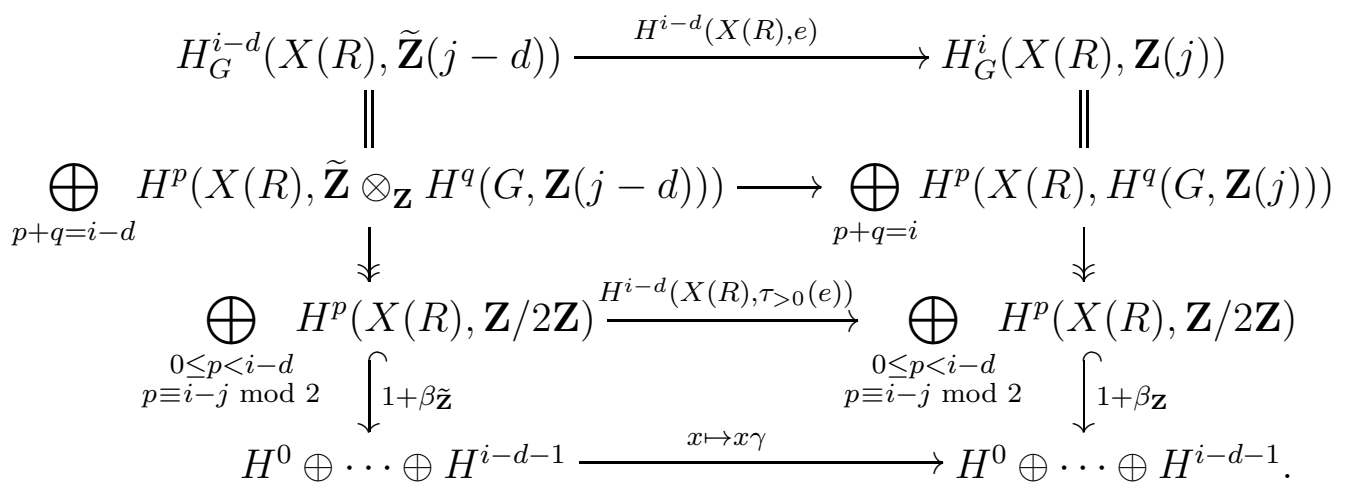

Here, for $\mathscr{F}=\widetilde{\mathbf{Z}}$ or $\mathscr{F}=\mathbf{Z}$, the label $1+\beta_{\mathscr{F}}$ denotes the sum of the twisted Bockstein homomorphisms $\beta_{\mathscr{F}}: H^{p} \rightarrow H^{p+1}$ (see \$1.2.3) for $p<i-d-1$ and of the identity maps $H^{p} \rightarrow H^{p}$ for $p<i-d$.

By Lemma 1.5, the bottom horizontal map is injective; therefore, so is the map $H^{i-d}\left(X(R), \tau_{>0}(e)\right)$. As the latter is an endomorphism of a finite-dimensional vector space, it must then be surjective. The proposition follows.

Remark 1.9. One can prove, although we shall not use this fact, that the map $H^{i-d}\left(X(R), \tau_{>0}(e)\right)$ in the above diagram is given by $x \mapsto x \widetilde{\gamma}+\beta_{\widetilde{\mathbf{Z}}}(x) \beta_{\widetilde{\mathbf{Z}}}(\widetilde{\gamma})$, where $\widetilde{\gamma} \in H^{0} \oplus H^{2} \oplus \cdots$ is such that $\gamma=\widetilde{\gamma}+\beta_{\widetilde{\mathbf{Z}}}(\widetilde{\gamma})$. In particular, the derived cup product with integral coefficients, in terms of the decomposition (1.29), is not just the sum of the individual cup product maps (in contrast with $\mathbf{Z} / 2 \mathbf{Z}$ coefficients; see (1.28)) ). 
1.4. Two Poincaré dualities. If $X$ is an irreducible, smooth and proper variety over $R$, of dimension $d$, there cannot be, in general, a duality between the equivariant cohomology groups $H_{G}^{i}(X(C), \mathbf{Z} / 2 \mathbf{Z})$ and $H_{G}^{2 d-i}(X(C), \mathbf{Z} / 2 \mathbf{Z})$, as the former group need not vanish when $i \gg 0$. The following proposition provides a substitute.

Proposition 1.10. Let $X$ be a smooth and proper variety over $R$, of pure dimension $d$. There is a canonical "trace" homomorphism

$$
H_{G}^{2 d}(X(C), X(R), \mathbf{Q} / \mathbf{Z}(d)) \rightarrow \mathbf{Q} / \mathbf{Z} ;
$$

together with cup product, it induces, for any finite $G$-module $M$, a perfect pairing of finite abelian groups

$$
H_{G}^{i}(X(C), X(R), M) \times H_{G}^{2 d-i}(X(C) \backslash X(R), \operatorname{Hom}(M, \mathbf{Q} / \mathbf{Z}(d))) \rightarrow \mathbf{Q} / \mathbf{Z}
$$

for any $i \in \mathbf{Z}$.

Proof. This is a reformulation of the semi-algebraic Poincaré duality (1.16) applied to $V=(X(C) \backslash X(R)) / G$ and to $\mathscr{G}=\mathscr{H}^{0}(G, M)$, in view of the isomorphisms (1.8) and (1.9), of the isomorphism or $_{V}=\mathscr{H}^{0}(G, \mathbf{Z}(d))$ and of the remark that by the properness of $X$, the cohomology of $V$ with complete supports coincides with the cohomology of $X(C) / G$ relative to $X(R)$ (see [Del91, Chapter II, Theorem 5.1], [DK82b, Theorem 2.3]).

Remarks 1.11. (i) When $X(R)=\varnothing$, Proposition 1.10 produces a canonical duality between the étale cohomology groups $H_{\text {ét }}^{i}(X, M)$ and $H_{\text {ét }}^{2 d-i}(X, \operatorname{Hom}(M, \mathbf{Q} / \mathbf{Z}(d)))$. Contrary to what happens for the étale cohomology of varieties over a $p$-adic field (for which see [Sai89, Lemma 2.9]), this duality does not result from the formal combination of Poincaré duality for the étale cohomology of $X_{C}$ with a duality in the Galois cohomology of $R$. Such a combination only yields a duality for the modified (à la Tate) étale hypercohomology of $X$ (loc. cit., Lemma 2.10).

(ii) If $M$ is a finitely generated $G$-module, applying Proposition 1.10 to $M / n M$ for all $n \geq 1$ yields, for any $i \in \mathbf{Z}$, a pairing that identifies the profinite completion of the finitely generated abelian group $H_{G}^{i}(X(C), X(R), M)$ with the Pontrjagin dual of the torsion abelian group $H_{G}^{2 d-i}(X(C) \backslash X(R), \operatorname{Hom}(M, \mathbf{Q} / \mathbf{Z}(d)))$.

Let us fix an irreducible, smooth and proper variety $X$ over $R$, of dimension $d$. We now have two Poincaré dualities at our disposal: one for the equivariant cohomology of $X(C) \backslash X(R)$, between the cohomological degrees $i$ and $2 d-i$ (see Proposition 1.10), and one for the cohomology of $X(R)$, between the cohomological degrees $i$ and $d-i$ (see (1.16) ). In the remainder of \$1.4, we show how to reconcile these two seemingly incompatible dualities in a single self-dual long exact sequence of cohomology groups.

Using the notation of $\$ 1.3 .1$, we first consider the localisation long exact sequence

$$
\cdots \stackrel{\delta_{i}}{\longrightarrow} H_{G}^{i}(X(C), X(R), \mathbf{Z} / 2 \mathbf{Z}) \stackrel{h_{i}}{\longrightarrow} H_{G}^{i}(X(C), \mathbf{Z} / 2 \mathbf{Z}) \stackrel{g_{i}}{\longrightarrow} H_{G}^{i}(X(R), \mathbf{Z} / 2 \mathbf{Z}) \stackrel{\delta_{i+1}}{\longrightarrow} \cdots
$$


The maps $f_{i}: H_{G, X(R)}^{i}(X(C), \mathbf{Z} / 2 \mathbf{Z}) \rightarrow H_{G}^{i}(X(C), \mathbf{Z} / 2 \mathbf{Z})$ and $g_{i} \circ f_{i}$ are injective, by Proposition [1.3 and Remark [1.6] (iii). The above sequence therefore remains exact if we replace $H_{G}^{i}(X(C), \mathbf{Z} / 2 \mathbf{Z})$ and $H_{G}^{i}(X(R), \mathbf{Z} / 2 \mathbf{Z})$ with $\operatorname{Coker}\left(f_{i}\right)$ and $\operatorname{Coker}\left(g_{i} \circ f_{i}\right)$, respectively. In view of Proposition 1.3 and Remark 1.6 (iii), we obtain, in this way, a long exact sequence

$$
\begin{aligned}
\stackrel{w_{i-1}}{\longrightarrow} H^{i-d} & \oplus \cdots \oplus H^{i-1} \stackrel{u_{i}}{\rightarrow} H_{G}^{i}(X(C), X(R), \mathbf{Z} / 2 \mathbf{Z}) \\
v_{i} & \rightarrow H_{G}^{i}(X(C) \backslash X(R), \mathbf{Z} / 2 \mathbf{Z}) \stackrel{w_{i}}{\longrightarrow} H^{i-d+1} \oplus \cdots \oplus H^{i} \stackrel{u_{i+1}}{\longrightarrow} \cdots
\end{aligned}
$$

in which the arrows may be described as follows. Let us denote by $e_{i}$ the restriction map $e_{i}: H_{G}^{i}(X(C), \mathbf{Z} / 2 \mathbf{Z}) \rightarrow H_{G}^{i}(X(C) \backslash X(R), \mathbf{Z} / 2 \mathbf{Z})$. We have $v_{i}=e_{i} \circ h_{i}$. The map $w_{i}$ is characterised by the property that $w_{i}\left(e_{i}\left(x^{\prime}\right)\right)$, for $x^{\prime} \in H_{G}^{i}(X(C), \mathbf{Z} / 2 \mathbf{Z})$, is obtained by decomposing $g_{i}\left(x^{\prime}\right)$ according to (1.26), then multiplying by $\gamma^{-1}$ and projecting onto $H^{i-d+1} \oplus \cdots \oplus H^{i}$. Finally, to compute $u_{i}(y)$ for $y \in H^{i-d} \oplus \cdots \oplus H^{i-1}$, we multiply $y$ by $\gamma$, project onto $H^{i-d} \oplus \cdots \oplus H^{i-1}$, view the result as an element of $H_{G}^{i-1}(X(R), \mathbf{Z} / 2 \mathbf{Z})$ via (1.26) and apply the connecting homomorphism $\delta_{i}$ of the localisation exact sequence.

Poincaré duality for $X(R)$, in the form of (1.16), yields a trace map $H^{d} \rightarrow \mathbf{Z} / 2 \mathbf{Z}$ (which is an isomorphism if and only if $X(R)$ is semi-algebraically connected). Let us denote by deg : $\bigoplus_{p \geq 0} H^{p} \rightarrow \mathbf{Z} / 2 \mathbf{Z}$ its composition with the projection map onto $H^{d}$. For every $i$, let us consider the pairing

$$
\left(H^{i-d+1} \oplus \cdots \oplus H^{i}\right) \times\left(H^{d-i} \oplus \cdots \oplus H^{2 d-i-1}\right) \rightarrow \mathbf{Z} / 2 \mathbf{Z}
$$

defined by $(x, y) \mapsto \operatorname{deg}(x y \gamma)$, where the products take place in the ring $\bigoplus_{p \geq 0} H^{p}$. This is a perfect pairing since the map $H^{p} \times H^{d-p} \rightarrow \mathbf{Z} / 2 \mathbf{Z}$ given by $(x, y) \mapsto \operatorname{deg}(x y)$ is a perfect pairing for every $p$ (see (1.16)) and $\gamma$ is invertible.

Theorem 1.12. For any irreducible, smooth and proper variety $X$ over $R$, the long exact sequence (1.46) is self-dual with respect to the perfect pairings (1.45) and (1.47). In other words, if $d$ denotes the dimension of $X$, then, for every integer $i$, the duals of $u_{i}, v_{i}, w_{i}$ with respect to these pairings are $w_{2 d-i}, v_{2 d-i}, u_{2 d-i}$, respectively.

Proof. For $x \in H_{G}^{i}(X(C), X(R), \mathbf{Z} / 2 \mathbf{Z})$ and $y \in H_{G}^{2 d-i}(X(C), X(R), \mathbf{Z} / 2 \mathbf{Z})$, we have $v_{i}(x) \smile y=x \smile y=x \smile v_{2 d-i}(y)$ in $H_{G}^{2 d}(X(C), X(R), \mathbf{Z} / 2 \mathbf{Z})$, hence $v_{2 d-i}$ is dual to $v_{i}$. Thus, to prove the theorem, we only have to show that

$$
x \smile u_{2 d-i}(y)=\operatorname{deg}\left(w_{i}(x) y \gamma\right)
$$

for any $i \geq 0$, any $x \in H_{G}^{i}(X(C) \backslash X(R), \mathbf{Z} / 2 \mathbf{Z})$ and any $y \in H^{d-i} \oplus \cdots \oplus H^{2 d-i-1}$. Let us fix $i, x$ and $y$. Let $x^{\prime} \in H_{G}^{i}(X(C), \mathbf{Z} / 2 \mathbf{Z})$ be the image of $x$ by the canonical section of $e_{i}$ given by Proposition 1.3. Let $x^{\prime \prime} \in H^{0} \oplus \cdots \oplus H^{i}$ denote the decomposition of $g_{i}\left(x^{\prime}\right)$ via (1.26). As $x^{\prime}$ is annihilated by the retraction (1.40), the product $x^{\prime \prime} \gamma^{-1}$ 
belongs to $H^{i-d+1} \oplus \cdots \oplus H^{i} \subseteq H^{0} \oplus \cdots \oplus H^{i}$. It follows that $w_{i}(x)=x^{\prime \prime} \gamma^{-1}$ and hence that

$$
\operatorname{deg}\left(w_{i}(x) y \gamma\right)=\operatorname{deg}\left(x^{\prime \prime} y\right)
$$

On the other hand, if $t^{\prime \prime} \in H^{d-i} \oplus \cdots \oplus H^{2 d-i-1}$ denotes the projection of $y \gamma$ and $t^{\prime} \in H_{G}^{2 d-i-1}(X(R), \mathbf{Z} / 2 \mathbf{Z})$ the element which corresponds to $t^{\prime \prime}$ via (1.26), we have

$$
\begin{aligned}
x \smile u_{2 d-i}(y) & =x^{\prime} \smile u_{2 d-i}(y)=x^{\prime} \smile \delta_{2 d-i}\left(t^{\prime}\right) \\
& =\delta_{2 d}\left(g_{i}\left(x^{\prime}\right) \smile t^{\prime}\right)=\operatorname{deg}\left(x^{\prime \prime} t^{\prime \prime} \gamma^{-1}\right),
\end{aligned}
$$

where the last equality follows from Lemma1.13 below (and the penultimate one from Bre97, Chapter II, Theorem 7.1 (b)]). Finally, as $x^{\prime \prime} \gamma^{-1}$ belongs to $H^{i-d+1} \oplus \cdots \oplus H^{i}$, we have $x^{\prime \prime} t^{\prime \prime} \gamma^{-1}=x^{\prime \prime} \gamma^{-1} t^{\prime \prime}=x^{\prime \prime} \gamma^{-1} y \gamma=x^{\prime \prime} y$; in view of (1.49) and (1.50), this completes the proof of (1.48) and hence of Theorem 1.12.

Lemma 1.13. Let $z \in H^{0} \oplus \cdots \oplus H^{2 d-1}$. The image of $z$ by the composed map

$$
H^{0} \oplus \cdots \oplus H^{2 d-1}=H_{G}^{2 d-1}(X(R), \mathbf{Z} / 2 \mathbf{Z}) \stackrel{\delta_{2 d}}{\longrightarrow} H_{G}^{2 d}(X(C), X(R), \mathbf{Z} / 2 \mathbf{Z})=\mathbf{Z} / 2 \mathbf{Z}
$$

where the two canonical isomorphisms come from (1.26) and from Proposition 1.10, is equal to $\operatorname{deg}\left(z \gamma^{-1}\right)$.

Proof. We may assume that $X(R) \neq \varnothing$. Let us consider the commutative diagram

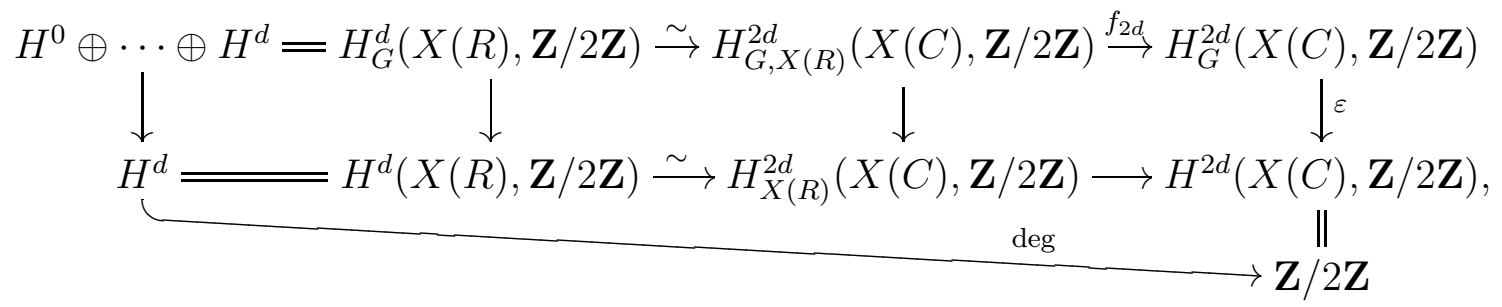

in which the leftmost vertical arrow is the projection map and the horizontal isomorphisms are the decomposition (1.26) and the equivariant purity isomorphism (1.18), with coefficients $\mathbf{Z} / 2 \mathbf{Z}$ or $\mathbf{Z} / 2 \mathbf{Z}[G]$. The leftmost square commutes by $\$ 1.2 .4$; the bottom triangle commutes as a consequence of the fact that $H^{d}$ is generated by classes supported on points. The hypothesis that $X(R) \neq \varnothing$ implies, by Proposition 1.10, that $H_{G}^{2 d}(X(C) \backslash X(R), \mathbf{Z} / 2 \mathbf{Z})=0$. It follows, by Proposition [1.3, that $f_{2 d}$ is an isomorphism and that its inverse is the map (1.40). From the definition of (1.40) and from the above diagram, we deduce the commutativity of the bottom square of 
the following diagram, whose vertical arrows are induced by (1.1):

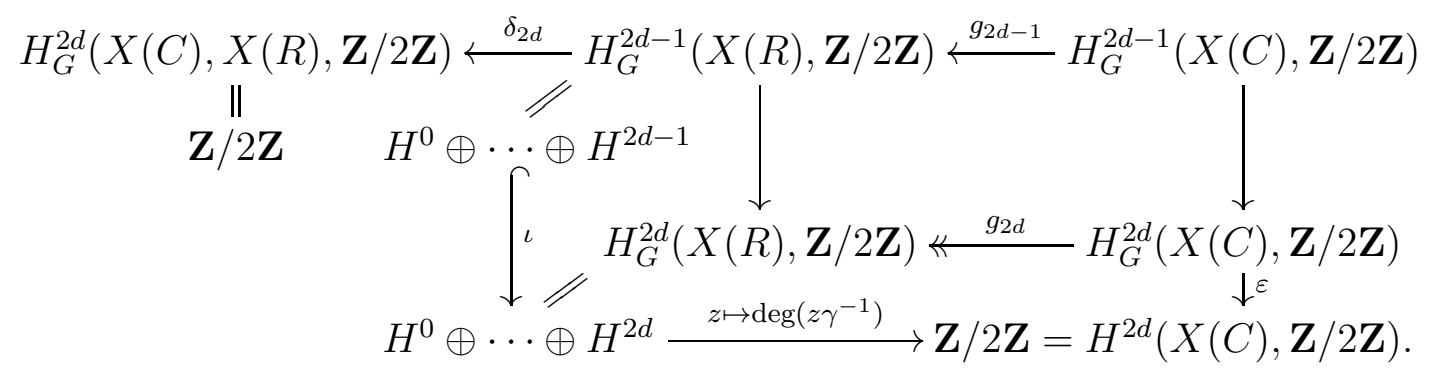

The map $g_{2 d}$ is surjective, as $H_{G}^{2 d+1}(X(C), X(R), \mathbf{Z} / 2 \mathbf{Z})=0$ (see $\left.\$ 1.1 .3\right)$. By $\$ 1.2 .4$, the map $\iota$ is the inclusion. As the top row and the right column are exact, we see that an element of $H^{0} \oplus \cdots \oplus H^{2 d-1}$ dies in the top left corner if and only if it dies in the bottom right corner. This proves the lemma.

1.5. A real Lefschetz hyperplane theorem. We shall need, in [BW18, §7.3], a Lefschetz hyperplane theorem for the equivariant Gysin map

$$
H_{G}^{i-2}(Y(C), \mathbf{Z}(j-1)) \rightarrow H_{G}^{i}(X(C), \mathbf{Z}(j))
$$

of a smooth ample hypersurface $Y \subset X$ in a smooth and projective variety $X$ over $R$ (see (1.21) ). The usual formulation is correct when $X(R)=\varnothing$, as we now check.

Proposition 1.14. Let $X$ be a smooth and projective variety over $R$, of dimension $d$. Let $Y \subset X$ be a smooth ample hypersurface. Let $j \in \mathbf{Z}$. If $X(R)=\varnothing$, the map (1.51) $i$ s bijective for any $i>d+1$ and is surjective for $i=d+1$.

Proof. Let $U=X \backslash Y$. It suffices to check that $H_{G}^{i}(U(C), M)=0$ for any $i>d$ and any finitely generated $G$-module $M$. This is true for $i \gg 0$, as $U(R)=\varnothing$ (see \$1.1.3). On the other hand, if $i>d$, we have $H^{i}(U(C), M) \otimes_{\mathbf{z}} \mathbf{Z}_{\ell}=H_{\text {ét }}^{i}\left(U_{C}, M \otimes_{\mathbf{z}} \mathbf{Z}_{\ell}\right)=0$ for every $\ell$, as $U_{C}$ is affine and $C$ is algebraically closed (see [Mil80, Chapter VI, Theorem 7.2]), hence $H^{i}(U(C), M)=0$. The desired vanishing then follows, thanks to the real-complex exact sequence (1.7), by a descending induction on $i$ in which $M$ is allowed to vary.

When $X(R) \neq \varnothing$, some condition on the real Gysin maps

$$
H^{p-1}(Y(R), \mathbf{Z} / 2 \mathbf{Z}) \rightarrow H^{p}(X(R), \mathbf{Z} / 2 \mathbf{Z})
$$

(see (1.13) ) must appear in the formulation of a Lefschetz hyperplane theorem as the ampleness of $Y$ has no effect on the injectivity or surjectivity of these maps. For example, the conclusion of Proposition 1.14 fails whenever $X(R) \neq \varnothing$ and $Y(R)=\varnothing$, since in this case $H_{G}^{i-2}(Y(C), \mathbf{Z}(i-1))=0$ and $H_{G}^{i}(X(C), \mathbf{Z}(i)) \neq 0$ for $i>2 d$, by $\$ 1.1 .3$ and (1.30). The next proposition generalises the surjectivity half of Proposition 1.14 when $X(R) \neq \varnothing$. 
Proposition 1.15. Let $X$ be a smooth and projective variety over $R$, of dimension d. Let $Y \subset X$ be a smooth ample hypersurface. Let $i, j \in \mathbf{Z}$ with $i \geq d+1$. If (1.52) is surjective for every $p \geq 1$ such that $p \equiv i-j \bmod 2$, then the image of (1.51) is

$$
\left\{\alpha \in H_{G}^{i}(X(C), \mathbf{Z}(j)) ;\left.\alpha\right|_{x}=0 \text { for all } x \in X(R)\right\},
$$

where $\left.\alpha\right|_{x} \in H_{G}^{i}(x, \mathbf{Z}(j))=H^{i}(G, \mathbf{Z}(j))$ denotes the restriction of $\alpha$ to $x$.

We start with a lemma.

Lemma 1.16. Let $U$ be an affine variety over $R$, of dimension $d$. For any $i>d$ and any $G$-module $M$, the restriction map $H_{G}^{i}(U(C), M) \rightarrow H_{G}^{i}(U(R), M)$ is an isomorphism.

Proof. Writing $M$ as the union of its finitely generated sub- $G$-modules, we see that we may assume $M$ to be finitely generated. The conclusion of the lemma, which holds for $i \gg 0$ (see \$1.1.3), then follows for $i>d$ by a descending induction, just as in the proof of Proposition 1.14, thanks to the real-complex exact sequence (1.7) and to the vanishing, for $i>d$, of the groups $H^{i}(U(C), M)$ (see the proof of Proposition 1.14) and $H^{i}(U(R), M)$ (see [Del91, Chapter II, Lemma 9.1]).

Proof of Proposition 1.15. Let $U=X \backslash Y$. The commutative diagram

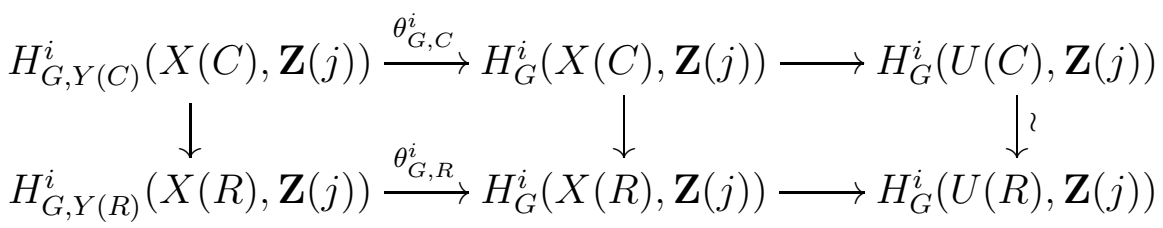

has exact rows and its rightmost vertical map is an isomorphism by Lemma 1.16

Let $\theta_{R}^{p}: H_{Y(R)}^{p}(X(R), \mathbf{Z} / 2 \mathbf{Z}) \rightarrow H^{p}(X(R), \mathbf{Z} / 2 \mathbf{Z})$ denote the forgetful map. Using the decompositions (1.30) and (1.32) (with $\mathscr{F}=\mathbf{Z}$ ), we see that when $i>d$, the map $\theta_{G, R}^{i}$ can be identified with the direct sum, over all $p \geq 0$ with $p \equiv i-j \bmod 2$, of the maps $\theta_{R}^{p}$. Thus, the maps $\theta_{R}^{p}$ for $p \geq 1$ such that $p \equiv i-j \bmod 2$ are all surjective if and only if the image of $\theta_{G, R}^{i}$ coincides with the set of $\alpha \in H_{G}^{i}(X(R), \mathbf{Z}(j))$ whose component in $H^{0}\left(X(R), H^{i}(G, \mathbf{Z}(j))\right)$ vanishes, i.e., with the set of $\alpha$ such that $\left.\alpha\right|_{x}=0$ for all $x \in X(R)$. The proposition then follows by a chase in the diagram (1.53), as $\theta_{R}^{p}$ and $\theta_{G, C}^{i}$ can be identified, respectively, with (1.52) and (1.51), via (1.13) and (1.21).

Remarks 1.17. (i) One can also check that for $i>d+1$, if (1.52) is injective for every $p \geq 1$ such that $p \equiv i-j \bmod 2$ and is surjective for every $p \geq 1$ such that $p \not \equiv i-j \bmod 2$, then (1.51) is injective. However, the proof is more involved and we shall not use this fact. 
(ii) In the setting of Lemma 1.16, Scheiderer [Sch94, Corollary 18.11] has shown, at least when $M$ is torsion, the stronger (and significantly more delicate) fact that $H_{G}^{i}(U(C), U(R), M)=0$ for all $i>d$.

1.6. Cycle classes and topological constraints. We fix, in this section, an integer $k$ and a smooth variety $X$ over $R$.

1.6.1. The equivariant cycle class map. For any irreducible closed subvariety $Y \subset X$ of codimension $k$, the equivariant fundamental class of $Y(C)$ in $X(C)$ is the image of 1 by the canonical isomorphism

$$
\mathbf{Z}=H_{G, Y(C)}^{2 k}(X(C), \mathbf{Z}(k))
$$

given by (1.21) when $Y$ is smooth, and which stems from (1.21) by dévissage in general (see [Mil80, Chapter VI, §9]). We let cl $(Y)$ denote its image in $H_{G}^{2 k}(X(C), \mathbf{Z}(k)$ ). For a codimension $k$ cycle $Y=\sum n_{i} Y_{i}$ on $X$, we let $\operatorname{cl}(Y)=\sum n_{i} \operatorname{cl}\left(Y_{i}\right)$. This assignment induces a homomorphism

$$
\mathrm{cl}: \mathrm{CH}^{k}(X) \rightarrow H_{G}^{2 k}(X(C), \mathbf{Z}(k))
$$

from the Chow group of codimension $k$ cycles on $X$, as was verified by Krasnov [Kra91, Proposition 2.1.1] when $R=\mathbf{R}$. The proof given in loc. cit. carries over to an arbitrary real closed field $R$ in view of the homotopy invariance of semialgebraic cohomology (see [DK82b, §6], [Del85]). The map cl is compatible with proper push-forwards (by functoriality of push-forward maps), with products (see [Kra91, Proposition 2.1.3]) and with pull-backs (loc. cit., Proposition 2.3.3).

1.6.2. The Borel-Haefliger cycle class map. For any irreducible closed subvariety $Y \subset X$ of codimension $k$, Borel and Haefliger [BH61] (and Delfs [Del91, Chapter III, Theorem 3.7] over an arbitrary real closed field) have proved the existence of a unique class in $H_{Y(R)}^{k}(X(R), \mathbf{Z} / 2 \mathbf{Z})$, called the fundamental class of $Y(R)$ in $X(R)$, whose restriction to $H_{Y^{0}(R)}^{k}\left(X^{0}(R), \mathbf{Z} / 2 \mathbf{Z}\right)$ is the fundamental class of $Y^{0}(R)$ in $X^{0}(R)$ in the sense of (1.13), where $Y^{0}$ (resp. $X^{0}$ ) denotes the complement, in $Y$ (resp. $X$ ), of the singular locus of $Y$. We denote by $\mathrm{cl}_{R}(Y)$ its image in $H^{k}(X(R), \mathbf{Z} / 2 \mathbf{Z})$. For a codimension $k$ cycle $Y=\sum n_{i} Y_{i}$ on $X$, we let $\mathrm{cl}_{R}(Y)=\sum n_{i} \mathrm{cl}_{R}\left(Y_{i}\right)$. This assignment induces a homomorphism

$$
\mathrm{cl}_{R}: \mathrm{CH}^{k}(X) \rightarrow H^{k}(X(R), \mathbf{Z} / 2 \mathbf{Z})
$$

(see [Sch95, Proposition 3.4, Remark 3.5]), which is compatible with proper pushforwards, products and pull-backs (e.g., as a consequence of the same property for the equivariant cycle class and of $\$ 1.6 .4$ below). We define $H_{\mathrm{alg}}^{k}(X(R), \mathbf{Z} / 2 \mathbf{Z})$ to be the image of the map (1.56). 
1.6.3. Topological constraints. Let us consider the composition

$$
H_{G}^{2 k}(X(C), \mathbf{Z}(k)) \rightarrow H_{G}^{2 k}(X(R), \mathbf{Z}(k)) \rightarrow \underset{\substack{0 \leq p \leq 2 k \\ p \equiv k \bmod 2}}{\bigoplus} H^{p}(X(R), \mathbf{Z} / 2 \mathbf{Z})
$$

of the restriction map from $X(C)$ to the $G$-invariant semi-algebraic subspace $X(R)$ with the map (1.31) induced by the decomposition (1.30). For $\alpha \in H_{G}^{2 k}(X(C), \mathbf{Z}(k))$, we let $\alpha_{p} \in H^{p}(X(R), \mathbf{Z} / 2 \mathbf{Z})$ denote the $p$ th coordinate of the image of $\alpha$ by (1.57).

The next theorem spells out the relationship between the equivariant cycle class and the Borel-Haefliger cycle class, as well as a topological constraint, expressed in terms of the Steenrod squares $\mathrm{Sq}^{i}: H^{k}(X(R), \mathbf{Z} / 2 \mathbf{Z}) \rightarrow H^{k+i}(X(R), \mathbf{Z} / 2 \mathbf{Z})$ (see [Eps66], [Ray68, Proposition 4.2]), which equivariant cycle classes must satisfy. We take the convention that $\mathrm{Sq}^{i}=0$ for $i<0$ and recall that $\mathrm{Sq}^{0}$ is the identity.

Theorem 1.18. Let $X$ be a smooth variety over $R$. Let $k$ be an integer. Let $Y$ be a cycle of codimension $k$ on $X$ and let $\alpha=\operatorname{cl}(Y) \in H_{G}^{2 k}(X(C), \mathbf{Z}(k))$. Then

$$
\alpha_{k+i}=\mathrm{Sq}^{i}\left(\mathrm{cl}_{R}(Y)\right)
$$

for every $i \in 2 \mathbf{Z}$.

This theorem is due to Kahn [Kah87] for Chern classes and to Krasnov [Kra94] in general (at least when $R=\mathbf{R}$ ). A more general result will be proved in \$1.6.4.

The statement of Theorem 1.18 motivates the following definition.

Definition 1.19. We denote by $H_{G}^{2 k}(X(C), \mathbf{Z}(k))_{0} \subseteq H_{G}^{2 k}(X(C), \mathbf{Z}(k))$ the subgroup consisting of those classes $\alpha$ which satisfy $\alpha_{k+i}=\operatorname{Sq}^{i}\left(\alpha_{k}\right)$ for every $i \in 2 \mathbf{Z}$.

Thanks to Theorem 1.18, we may now view the equivariant cycle class map as a map cl : $\mathrm{CH}^{k}(X) \rightarrow H_{G}^{2 k}(X(C), \mathbf{Z}(k))_{0}$. As a notable consequence of Theorem 1.18, the Borel-Haefliger cycle class map factors through the equivariant cycle class map; namely, it coincides with the composition of cl with the map

$$
H_{G}^{2 k}(X(C), \mathbf{Z}(k))_{0} \rightarrow H^{k}(X(R), \mathbf{Z} / 2 \mathbf{Z})
$$

defined by $\alpha \mapsto \alpha_{k}$.

Remarks 1.20. (i) Let $d=\operatorname{dim}(X)$. The condition appearing in Definition 1.19 takes a particularly simple form for $k=d-1$ since $H^{p}(X(R), \mathbf{Z} / 2 \mathbf{Z})=0$ for $p \geq d+1$ : the group $H_{G}^{2 d-2}(X(C), \mathbf{Z}(d-1))_{0}$ consists of those $\alpha \in H_{G}^{2 d-2}(X(C), \mathbf{Z}(d-1))$ such that $\alpha_{p}=0$ for all $p<d-1$.

(ii) Let us fix $\alpha \in H_{G}^{2 k}(X(C), \mathbf{Z}(k))$ and denote by $\bar{\alpha} \in H_{G}^{2 k}(X(R), \mathbf{Z} / 2 \mathbf{Z})$ the class obtained by restricting to $X(R)$ and by reducing the coefficients modulo 2 . For $p \in \mathbf{Z}$, let $\bar{\alpha}_{p} \in H^{p}(X(R), \mathbf{Z} / 2 \mathbf{Z})$ be the $p$ th coordinate of $\bar{\alpha}$ in the decomposition (1.26). Then $\alpha \in H_{G}^{2 k}(X(C), \mathbf{Z}(k))_{0}$ if and only if $\bar{\alpha}_{k+i}=\operatorname{Sq}^{i}\left(\bar{\alpha}_{k}\right)$ for every $i \in \mathbf{Z}$. This 
follows from $\$ 1.2 .3$ and from the Adem relation $\mathrm{Sq}^{2 m+1}=\beta_{\mathbf{Z}} \circ \mathrm{Sq}^{2 m}$ (for which we refer the reader to Ray68, Proposition 4.2]).

(iii) For any even $k$ and any $\alpha \in H_{G}^{2 k}(X(C), \mathbf{Z}(k))_{0}$, the class $\alpha_{k}^{2}$ belongs to the image of the natural map $H^{2 k}(X(R), \mathbf{Z}) \rightarrow H^{2 k}(X(R), \mathbf{Z} / 2 \mathbf{Z})$. Indeed, so does $\alpha_{2 k}$ by its very definition, and we have $\alpha_{k}^{2}=\mathrm{Sq}^{k}\left(\alpha_{k}\right)=\alpha_{2 k}$. As noted by Krasnov [Kra94, Remark 4.8], this observation, in the case of equivariant cycle classes, was known to Akbulut and King (see [AK93, Theorem A (b)]).

1.6.4. Compatibility with cup products, pull-backs, push-forwards. Using \$1.2.3, the formula (1.28) and the Cartan formula (see [MS74, p. 91]), it is easy to check that Definition 1.19 is compatible with cup products, in the sense that for any $k$ and $\ell$, cup product induces the horizontal arrows of a commutative square

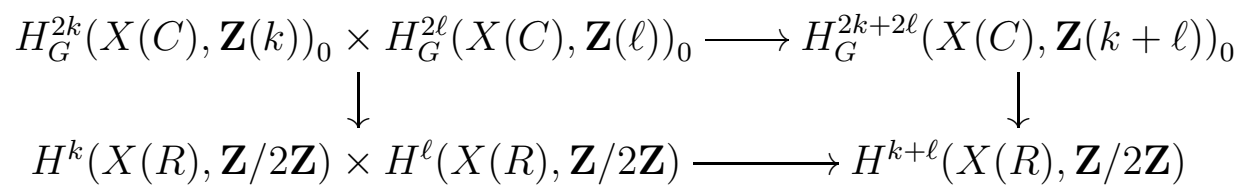

whose vertical arrows are the maps (1.58). In addition, the topological constraints of Definition 1.19 and the map (1.58) are obviously compatible with pull-backs. We verify, in Theorem 1.21 below, that they are also compatible with push-forwards along proper maps. The proof of this fact does not depend on Theorem 1.18. Applying Theorem [1.21 with $\ell=0$ therefore gives an independent proof of Theorem 1.18 .

Theorem 1.21. Let $X, Y$ be smooth, irreducible varieties over $R$. Let $f: Y \rightarrow X$ be a proper morphism. Let $\ell$ be an integer. Let $k=\ell+\operatorname{dim}(X)-\operatorname{dim}(Y)$. Then

$$
f_{*}\left(H_{G}^{2 \ell}(Y(C), \mathbf{Z}(\ell))_{0}\right) \subseteq H_{G}^{2 k}(X(C), \mathbf{Z}(k))_{0},
$$

where $f_{*}: H_{G}^{2 \ell}(Y(C), \mathbf{Z}(\ell)) \rightarrow H_{G}^{2 k}(X(C), \mathbf{Z}(k))$ is the push-forward homomorphism defined in (1.22). Moreover, the square

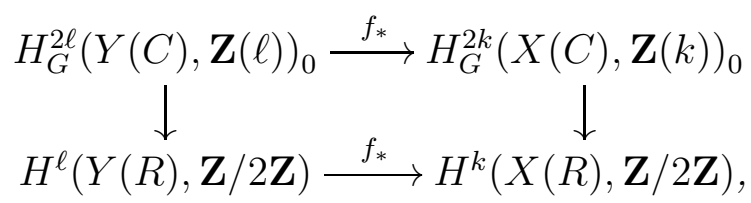

whose lower horizontal arrow is the push-forward map defined in (1.14) and whose vertical arrows are the maps (1.58) associated with $Y$ and with $X$, is commutative.

In view of Remark 1.20 (ii), Theorem 1.21 follows from Proposition 1.22 below. 
Proposition 1.22. Let $f: Y \rightarrow X$ be a proper morphism between smooth, irreducible varieties over $R$. Let $n$ be an integer. Let $m=n+2 \operatorname{dim}(X)-2 \operatorname{dim}(Y)$. The square

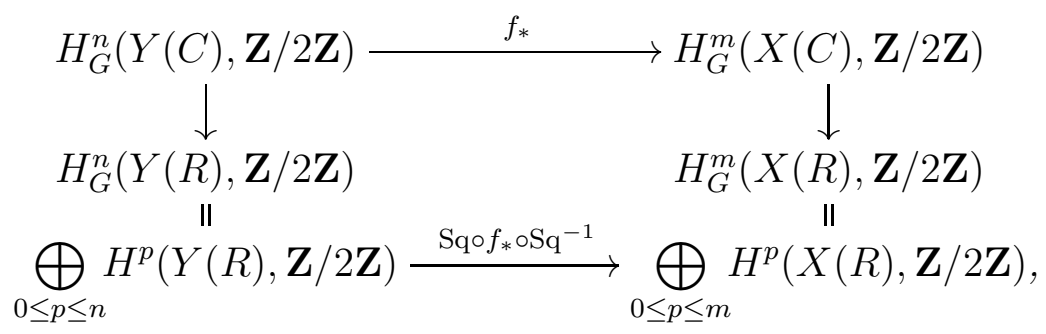

whose vertical maps are the restriction maps composed with the decompositions (1.26), commutes. In the lower row of this diagram, the symbol $f_{*}$ denotes the direct sum of the push-forward maps

$$
f_{*}: H^{p}(Y(R), \mathbf{Z} / 2 \mathbf{Z}) \rightarrow H^{p+\operatorname{dim}(X)-\operatorname{dim}(Y)}(X(R), \mathbf{Z} / 2 \mathbf{Z})
$$

over all $p \geq 0$ (see (1.14)), while $\mathrm{Sq}=\mathrm{Sq}^{0}+\mathrm{Sq}^{1}+\mathrm{Sq}^{2}+\ldots$ is viewed as an automorphism of the graded rings $\bigoplus_{0 \leq p \leq n} H^{p}(Y(R), \mathbf{Z} / 2 \mathbf{Z})$ and $\bigoplus_{0 \leq p \leq m} H^{p}(X(R), \mathbf{Z} / 2 \mathbf{Z})$, and $\mathrm{Sq}^{-1}$ stands for its inverse.

Proof. As a consequence of $\$ 1.2 .5$ and of the formula (1.28), the diagram

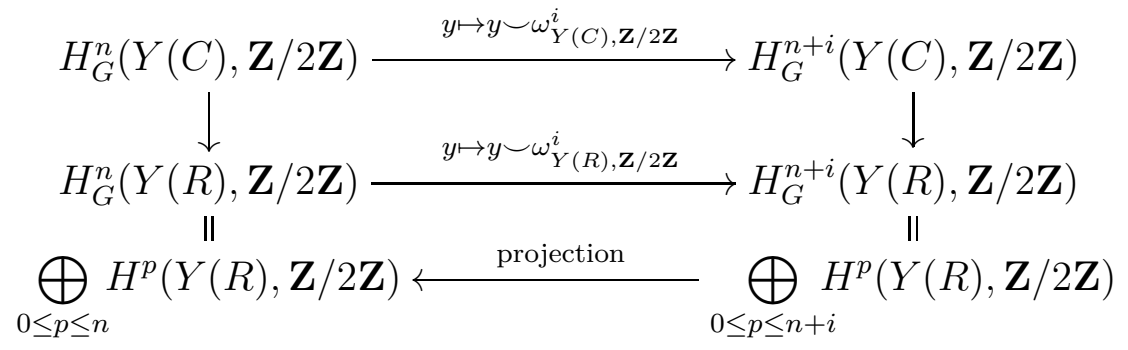

commutes for any $i \geq 0$ (and similarly with $X$ instead of $Y$ ). In view of the projection

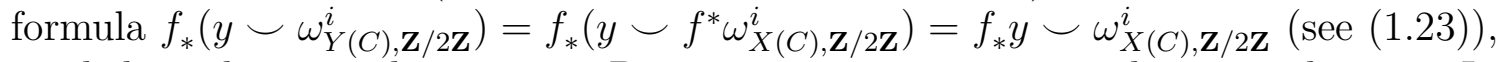
we deduce that in order to prove Proposition 1.22 , we may replace $n$ with $n+i$. In particular, we may, and will, assume that $n>2 \operatorname{dim}(Y)+1$. 
Let us then consider the commutative diagram (without the dotted arrows)

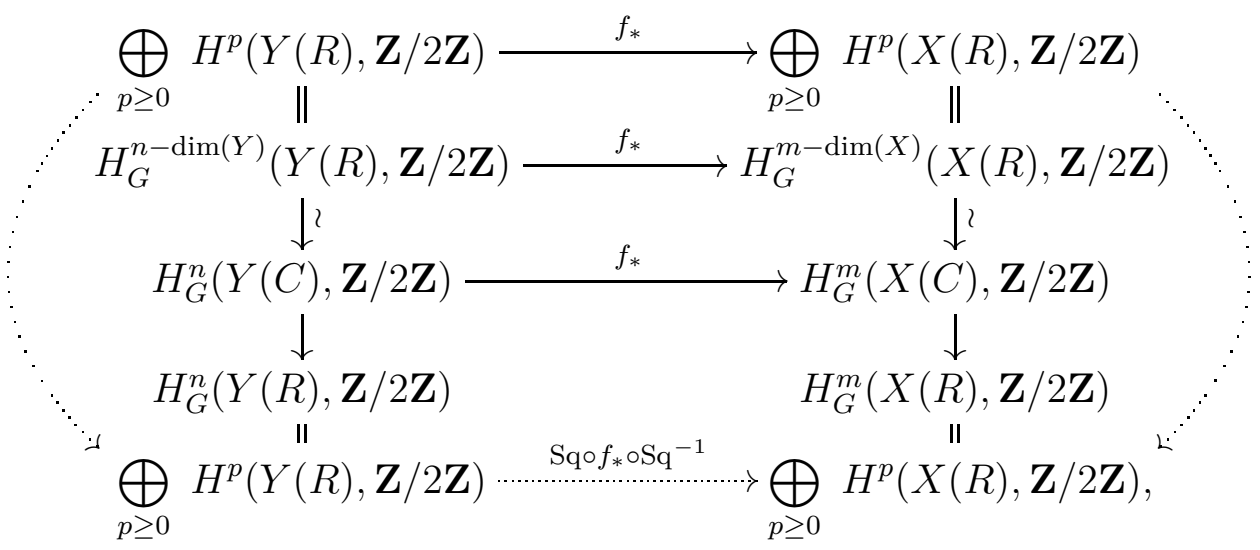

in which the upper horizontal $f_{*}$ denotes the direct sum of the maps (1.59) over all $p \geq 0$ (see $\$ 1.2 .6$ for the commutativity of the top square) and the vertical arrows of the middle square are the Gysin maps associated with the inclusions $Y(R) \subseteq Y(C)$ and $X(R) \subseteq X(C)$ (see (1.20) ). As we have seen in the proof of Lemma 1.5, these two Gysin maps are isomorphisms since $n>2 \operatorname{dim}(Y)+1$ and $m>2 \operatorname{dim}(X)+1$, and the bent arrows which make the diagram commute are given by multiplication by the classes $\gamma_{Y} \in \bigoplus_{p \geq 0} H^{p}(Y(R), \mathbf{Z} / 2 \mathbf{Z})$ and $\gamma_{X} \in \bigoplus_{p \geq 0} H^{p}(X(R), \mathbf{Z} / 2 \mathbf{Z})$ that one obtains by applying Definition 1.4 to $Y$ and to $X$. To prove that the diagram remains commutative with the horizontal dotted arrow, it therefore suffices to check that

$$
\operatorname{Sq}\left(f_{*}\left(\operatorname{Sq}^{-1}(y)\right)\right)=\gamma_{X} f_{*}\left(\gamma_{Y}^{-1} y\right)
$$

for any $y \in \bigoplus_{p \geq 0} H^{p}(Y(R), \mathbf{Z} / 2 \mathbf{Z})$, or, equivalently, that

$$
f_{*}\left(\mathrm{Sq}^{-1}(y) \mathrm{Sq}^{-1}\left(\gamma_{Y}\right)\right)=\mathrm{Sq}^{-1}\left(f_{*} y\right) \mathrm{Sq}^{-1}\left(\gamma_{X}\right)
$$

for any $y \in \bigoplus_{p \geq 0} H^{p}(Y(R), \mathbf{Z} / 2 \mathbf{Z})$.

This last equality is a particular case of the relative variant of Wu's theorem due to Atiyah and Hirzebruch [AH61. Namely, when $R=\mathbf{R}$ and $X$ and $Y$ are proper, this is op. cit., Satz 3.2 applied to $\lambda=\mathrm{Sq}^{-1}$; indeed, with the notation of loc. cit., one has $\mathrm{Wu}(\mathrm{Sq}, Y)=\mathrm{Sq}^{-1}\left(\gamma_{Y}\right)$ and $\mathrm{Wu}(\mathrm{Sq}, X)=\operatorname{Sq}^{-1}\left(\gamma_{X}\right)$, according to Thom's formula [MS74, p. 91] and to Remark 1.6 (i) (see [Kra94, Theorem 2.1]). When $R=\mathbf{R}$ but $X$ and $Y$ need not be proper, the proof given in [AH61, §3.4] goes through verbatim once one remarks that if $i$ denotes the composition of a closed embedding $Y(\mathbf{R}) \subset \mathbf{R}^{N}$ given by Whitney's theorem (see Hir94, Chapter 2, Theorem 2.14]) with an embedding of $\mathbf{R}^{N}$ into the $N$-dimensional sphere $\mathbf{S}^{N}$, the map $(f, i): Y(\mathbf{R}) \rightarrow X(\mathbf{R}) \times \mathbf{S}^{N}$ is a closed embedding. 
By a spreading out argument entirely similar to the one used in [DK82a, §7], one can deduce the validity of (1.60) over an arbitrary real closed field $R$ from the validity of (1.60) for all $X, Y, f, y$ defined over $\mathbf{R}$. (The point is that (1.60) is of a purely cohomological nature. Spreading out allows one to deduce cohomological statements over arbitrary real closed fields from the same statements over $\mathbf{R}$. For statements on algebraic cycles, the situation is quite different, as we will see in [BW18, §9].)

To establish (1.60) without spreading out, one can also, in the projective case, apply the Riemann-Roch theorem of Panin and Smirnov [Pan04, Theorem 2.5.4] to semi-algebraic cohomology with $\mathbf{Z} / 2 \mathbf{Z}$ coefficients.

\section{The ReAl integral Hodge Conjecture}

We formulate, in this section, a real analogue of the integral Hodge conjecture.

2.1. Reminders on the complex integral Hodge conjecture. Let $k \geq 0$. Let $X$ be a smooth and proper variety of pure dimension $d$ over $\mathbf{C}$.

A class $\alpha \in H^{2 k}(X(\mathbf{C}), \mathbf{Z}(k))$ is Hodge if its image in $H^{2 k}(X(\mathbf{C}), \mathbf{C})$ is of type $(k, k)$ in the Hodge decomposition. Let $\operatorname{Hdg}^{2 k}(X(\mathbf{C}), \mathbf{Z}(k)) \subseteq H^{2 k}(X(\mathbf{C}), \mathbf{Z}(k))$ denote the subgroup of Hodge classes. Classes of algebraic cycles belong to $\operatorname{Hdg}^{2 k}(X(\mathbf{C}), \mathbf{Z}(k))$. By definition, the integral Hodge conjecture for codimension $k$ cycles on $X$ holds if and only if the induced map $\mathrm{CH}^{k}(X) \rightarrow \operatorname{Hdg}^{2 k}(X(\mathbf{C}), \mathbf{Z}(k))$ is surjective.

The integral Hodge conjecture holds for $k=0$ or $k \geq d$ (trivial) and for $k=1$ (this is the Lefschetz $(1,1)$ theorem). For all other values of $k$ and $d$, it fails in general. The first counterexamples were discovered by Atiyah and Hirzebruch [AH62]. These are counterexamples for $k=2$ and $d \geq 7$. In the case of 1-cycles, Kollár [BCC92] has shown that the integral Hodge conjecture fails for very general hypersurfaces of degree $\delta$ in $\mathbf{P}_{\mathbf{C}}^{4}$ for some $\delta$. The smallest value of $\delta$ known to yield a counterexample is 48 (see [Tot13, §5]), though it is expected that any $\delta \geq 6$ should yield one: indeed, Griffiths and Harris [GH85, p. 32] conjecture that if $\delta \geq 6$, the degree of any curve on a very general hypersurface of degree $\delta$ in $\mathbf{P}_{\mathbf{C}}^{4}$ is a multiple of $\delta$.

By blowing up $\mathbf{P}_{\mathbf{C}}^{d}$ along a very general hypersurface of degree $\delta$ in $\mathbf{P}_{\mathbf{C}}^{4} \subset \mathbf{P}_{\mathbf{C}}^{d}$, one finds counterexamples to the integral Hodge conjecture among rational varieties for all $k \in\{3, \ldots, d-2\}$ (see [SV05, p. 113]). On the other hand, for $k \in\{2, d-1\}$, the integral Hodge conjecture for codimension $k$ cycles on $X$ is a birational invariant (see [Voi07, Lemma 15]) and in particular it holds for rational varieties. The integral Hodge conjecture can nevertheless fail for $k=2$ among rationally connected varieties [CTV12, Théorème 1.3], even for rationally connected fourfolds [Sch18, Corollary 1.6], although it holds for smooth cubic fourfolds [Voi07, Theorem 18]. For $k=d-1$, the following question is open:

Question 2.1 (Voisin [Voi07, Question 16]). Let X be a smooth, proper, rationally connected variety over $\mathbf{C}$. Does $X$ satisfy the integral Hodge conjecture for 1-cycles? 
Voisin [Voi06, Theorem 2] proves that a complex projective threefold satisfies the integral Hodge conjecture if it is uniruled or Calabi-Yau[1. This answers Question 2.1 in the affirmative when $\operatorname{dim}(X)=3$. In higher dimensions, there are partial results for Fano varieties (see [HV11, Flo13]). In addition, using a theorem of Schoen [Sch98], Voisin [Voi13, Theorem 1.6] shows that the Tate conjecture for all surfaces over finite fields would imply a positive answer to Question 2.1.

2.2. The real formulation. Let $k \geq 0$. Let $X$ be a smooth and proper variety over R. According to Theorem 1.18, the image of the equivariant cycle class map $\mathrm{CH}^{k}(X) \rightarrow H_{G}^{2 k}(X(\mathbf{C}), \mathbf{Z}(k))$ is contained in the subgroup $H_{G}^{2 k}(X(\mathbf{C}), \mathbf{Z}(k))_{0}$ introduced in Definition 1.19, As recalled in \$2.1, it is also contained in the inverse image $\operatorname{Hdg}_{G}^{2 k}(X(\mathbf{C}), \mathbf{Z}(k)) \subseteq H_{G}^{2 k}(X(\mathbf{C}), \mathbf{Z}(k))$ of $\operatorname{Hdg}^{2 k}(X(\mathbf{C}), \mathbf{Z}(k))$ by the natural map $H_{G}^{2 k}(X(\mathbf{C}), \mathbf{Z}(k)) \rightarrow H^{2 k}(X(\mathbf{C}), \mathbf{Z}(k))$. Hence we obtain a map

$$
\mathrm{cl}: \mathrm{CH}^{k}(X) \rightarrow \operatorname{Hdg}_{G}^{2 k}(X(\mathbf{C}), \mathbf{Z}(k))_{0}
$$

to the subgroup $\operatorname{Hdg}_{G}^{2 k}(X(\mathbf{C}), \mathbf{Z}(k))_{0}=\operatorname{Hdg}_{G}^{2 k}(X(\mathbf{C}), \mathbf{Z}(k)) \cap H_{G}^{2 k}(X(\mathbf{C}), \mathbf{Z}(k))_{0}$ of those classes in $H^{2 k}(X(\mathbf{C}), \mathbf{Z}(k))$ which satisfy both the Hodge condition and the topological condition.

Definition 2.2. Let $k \geq 0$. Let $X$ be a smooth and proper variety over $\mathbf{R}$. We say that the real integral Hodge conjecture for codimension $k$ cycles on $X$ holds if the map (2.1) is surjective.

Let us stress that this property can fail, just as the integral Hodge conjecture can fail for varieties over $\mathbf{C}$ (see \$2.1). We shall provide examples of geometrically connected varieties over $\mathbf{R}$ for which it fails in \$4.3, see also Example 2.5 below.

We note that if $H^{q}\left(X, \Omega_{X}^{p}\right)=0$ for all $p, q$ such that $p+q=2 k,(p, q) \neq(k, k)$, then $\operatorname{Hdg}_{G}^{2 k}(X(\mathbf{C}), \mathbf{Z}(k))_{0}=H_{G}^{2 k}(X(\mathbf{C}), \mathbf{Z}(k))_{0}$ and the formulation of the real integral Hodge conjecture makes sense over an arbitrary real closed field (where Hodge theory is not readily available).

Definition 2.3. Let $k \geq 0$. Let $X$ be a smooth and proper variety over a real closed field $R$. Assume that $H^{q}\left(X, \Omega_{X}^{p}\right)=0$ whenever $p+q=2 k$ and $(p, q) \neq(k, k)$. We say that the real integral Hodge conjecture for codimension $k$ cycles on $X$ holds if the equivariant cycle class map cl : $\mathrm{CH}^{k}(X) \rightarrow H_{G}^{2 k}(X(C), \mathbf{Z}(k))_{0}$ is surjective.

Remarks 2.4. (i) Considering equivariant cohomology à la Bredon, rather than à la Borel, leads to a factorisation $\mathrm{CH}^{k}(X) \rightarrow H_{\mathrm{Br}}^{2 k, k}(X(\mathbf{C}), \underline{\mathbf{Z}}) \rightarrow H_{G}^{2 k}(X(\mathbf{C}), \mathbf{Z}(k))$ of

\footnotetext{
${ }^{1}$ We mean Calabi-Yau in the sense that $K_{X} \simeq \mathscr{O}_{X}$ and $H^{1}\left(X, \mathscr{O}_{X}\right)=H^{2}\left(X, \mathscr{O}_{X}\right)=0$. Totaro has announced a proof of the integral Hodge conjecture for complex projective threefolds $X$ such that $K_{X} \simeq \mathscr{O}_{X}$. Abelian threefolds are dealt with in [Gra04, Corollary 3.1.9]. The hypothesis that $K_{X} \simeq \mathscr{O}_{X}$ cannot be weakened to $K_{X}$ being torsion in view of [BO18, Theorem 0.1].
} 
the equivariant cycle class map cl (see dSLF09, $\S \S 1-2]$ ). It would be interesting to determine what additional constraints on the image of $\mathrm{cl}$, if any, result from this.

(ii) By a norm argument, the map (2.1) tensored with $\mathbf{Q}$ is surjective if $X_{\mathbf{C}}$ satisfies the Hodge conjecture. Thus, there would be no point in formulating a real variant of the Hodge conjecture with rational coefficients.

An intriguing feature of the real integral Hodge conjecture, one with no analogue in the complex setting, is the existence of canonical "constant" cohomology classes. Namely, let $X$ be a smooth and proper variety over a real closed field $R$ and let $k \geq 0$ be even, so that $\mathbf{Z}(2 k)=\mathbf{Z}(k)$. We can view $\omega^{2 k} \in H_{G}^{2 k}(X(C), \mathbf{Z}(2 k)$ ) (see \$1.1.2) as an element of $H_{G}^{2 k}(X(C), \mathbf{Z}(k))$. This class is Hodge if $R=\mathbf{R}$, being torsion, and it belongs to $H_{G}^{2 k}(X(C), \mathbf{Z}(k))_{0}$ if (and only if) $X(R)=\varnothing$ (see $\$ 1.2 .5$ ). If $X(R)=\varnothing$, the integral Hodge conjecture on $X$ therefore implies that $\omega^{2 k}$ is algebraic, i.e., is the class of an algebraic cycle. Determining when $\omega^{2 k}$ is algebraic is an interesting problem in its own right.

Example 2.5. Let $X$ be the smooth projective anisotropic quadric of dimension $n$ over $R$. By [Ben16, Proposition 3.3, Proposition 3.5] and by comparison with 2-adic cohomology, the class $\omega^{4}$ is algebraic if and only if -1 is a sum of 7 squares in the function field $R(X)$. By Pfister [Pfi65, Satz 5], such is the case if and only if $n \leq 6$.

In this example, the real integral Hodge conjecture for codimension 2 cycles on $X$ fails if $n \geq 7$. This can be compared with [CTV12, Théorème 1.3]. In constrast, the following question is open.

Question 2.6. Does there exist a smooth and proper variety of odd dimension $d$, over a real closed field $R$, such that $X(R)=\varnothing$ and $\omega^{2 d-2}$ is not algebraic?

Remarks 2.7. (i) Let $X$ be a smooth and proper variety over $\mathbf{R}$ and let $k \geq 0$. If the restriction of the map (1.58) to the subgroup $\operatorname{Hdg}_{G}^{2 k}(X(\mathbf{C}), \mathbf{Z}(k))_{0}$ is not surjective, then $H_{\text {alg }}^{k}(X(\mathbf{R}), \mathbf{Z} / 2 \mathbf{Z}) \neq H^{k}(X(\mathbf{R}), \mathbf{Z} / 2 \mathbf{Z})$. As far as we are aware, this simple remark explains all of the examples that appear in the literature of real varieties $X$ and integers $k$ such that $H_{\text {alg }}^{k}(X(\mathbf{R}), \mathbf{Z} / 2 \mathbf{Z}) \neq H^{k}(X(\mathbf{R}), \mathbf{Z} / 2 \mathbf{Z})$. We give an example of a different kind in \$4.3 (see Example 4.7); such an example necessarily underlies a defect of the real integral Hodge conjecture for codimension $k$ cycles on $X$.

(ii) Let us justify the assertion made in (i) about the existing literature. Examples with $k=1$, such as those of [Ris82] and [Sil82], fall under the scope of Remark 2.7](i) since the map (2.1) is surjective when $k=1$ according to Proposition 2.8 below. Next, the examples given in [AK85, Theorem 6.9] and in [Kuc85, Theorem 3.1] are examples in which even the map (1.58) is not surjective. Indeed, these examples only depend on two properties of the subgroups $H_{\mathrm{alg}}^{k}(X(\mathbf{R}), \mathbf{Z} / 2 \mathbf{Z})$ : their stability under cup products, pull-backs and proper push-forwards, and the fact that $H_{\text {alg }}^{0}(X(\mathbf{R}), \mathbf{Z} / 2 \mathbf{Z}) \neq H^{0}(X(\mathbf{R}), \mathbf{Z} / 2 \mathbf{Z})$ when $X$ is connected while $X(\mathbf{R})$ is not; 
as it turns out, the images of (1.58) form a system of subgroups that enjoy these properties as well (see \$1.6.4). The example of [BD84 is an example of a class in $H^{2}(X(\mathbf{R}), \mathbf{Z} / 2 \mathbf{Z})$ whose square cannot be lifted to $H^{4}(X(\mathbf{R}), \mathbf{Z})$ (see [Tei95, especially the end of $\S 2$ ); by Remark 1.20 (iii), the map (1.58) again fails to be surjective in this case. Finally, in the examples furnished by [Kuc96, Theorem 2.1], the map (1.58) fails once more to be surjective, as we explain in Remark 2.12 below.

2.3. First positive results. On a smooth and proper variety $X$ of dimension $d$ over a real closed field $R$, the real integral Hodge conjecture for codimension $k$ cycles holds if $k=0$ or $k>d$. For $k=0$, this is a trivial assertion; for $k>d$, the restriction map $H_{G}^{2 k}(X(C), \mathbf{Z}(k)) \rightarrow H_{G}^{2 k}(X(R), \mathbf{Z}(k)$ ) is injective (see \$1.1.3) with kernel $H_{G}^{2 k}(X(C), \mathbf{Z}(k))_{0}$, so that the target of (2.1) vanishes. Let us now consider, in $\$ 2.3 .1$ and $\$ 2.3 .2$ below, the more interesting cases where $k=1$ or $k=d$.

2.3.1. Divisors. It was observed by Krasnov that the Lefschetz $(1,1)$ theorem holds in the real setting as well. The topological constraints provided by Theorem 1.18 do not play any role here, as $H_{G}^{2}(X(C), \mathbf{Z}(1))_{0}=H_{G}^{2}(X(C), \mathbf{Z}(1))$.

Proposition 2.8 (Krasnov). Any smooth and proper variety over $\mathbf{R}$ satisfies the real integral Hodge conjecture for codimension 1 cycles.

This can be checked by mimicking the usual proof of the Lefschetz $(1,1)$ theorem and noting that the exponential short exact sequence on $X(\mathbf{C})$ is $G$-equivariant. We refer the reader to [Kra91, Proposition 1.3.1], [MvH98, Proposition 3.2], vH00a, Chapter IV, Theorem 4.1]. Over a real closed field, one has the following substitute:

Proposition 2.9. Let $X$ be a smooth variety over a real closed field $R$. The cokernel of the equivariant cycle class map cl : $\operatorname{Pic}(X) \rightarrow H_{G}^{2}(X(C), \mathbf{Z}(1))$ is torsion-free. Assume, moreover, that $X$ is proper and that $H^{2}\left(X, \mathscr{O}_{X}\right)=0$. Then $X$ satisfies the real integral Hodge conjecture for codimension 1 cycles in the sense of Definition 2.3.

Proof. Let $E=$ Coker $\left(\mathrm{cl}: \operatorname{Pic}(X) \rightarrow H_{G}^{2}(X(C), \mathbf{Z}(1))\right)$. Let $\operatorname{Br}(X)=H_{\text {ét }}^{2}\left(X, \mathbf{G}_{\mathrm{m}}\right)$ and $T(\operatorname{Br}(X))=\operatorname{Hom}(\mathbf{Q} / \mathbf{Z}, \operatorname{Br}(X))$. Recall, from \$1.1.1, the canonical identification $H_{G}^{2}(X(C), \mathbf{Z}(1)) \otimes_{\mathbf{Z}} \widehat{\mathbf{Z}}=H_{\text {ét }}^{2}(X, \widehat{\mathbf{Z}}(1))$, and consider the commutative diagram

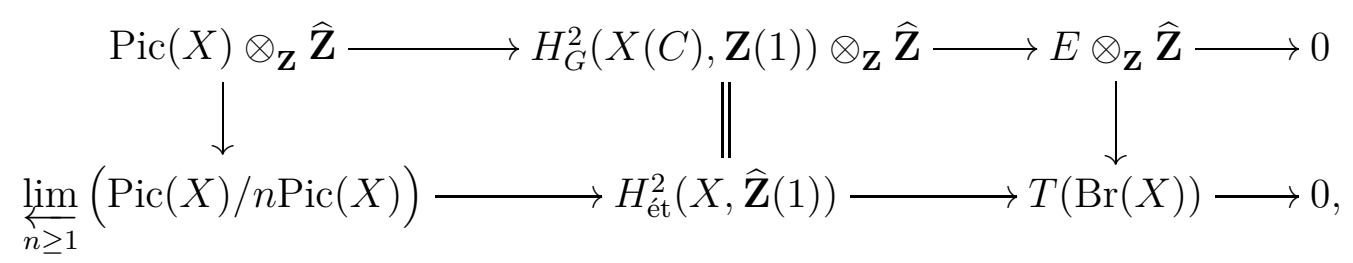

in which the bottom row comes from the Kummer exact sequence in étale cohomology and the vertical map on the right is determined by the rest of the diagram. The zigzag map $\lim _{\longleftarrow} \operatorname{Pic}(X) / n \operatorname{Pic}(X) \rightarrow E \otimes_{\mathbf{Z}} \widehat{\mathbf{Z}}$ vanishes since its composition 
with the projection $E \otimes_{\mathbf{Z}} \widehat{\mathbf{Z}} \rightarrow E / n E$ vanishes for every $n$ while $E$ is a finitely generated abelian group. The vertical map on the right is therefore an isomorphism. As the abelian group $T(\operatorname{Br}(X))$ is torsion-free and as $E$ is finitely generated, it follows that $E$ is torsion-free. If $X$ is proper and satisfies $H^{2}\left(X, \mathscr{O}_{X}\right)=0$, then $\mathrm{NS}\left(X_{C}\right) \otimes_{\mathbf{Z}} \mathbf{Q}_{\ell}=H_{\text {ét }}^{2}\left(X_{C}, \mathbf{Q}_{\ell}\right)$ for any $\ell$ (by Hodge theory if $C=\mathbf{C}$, by the Lefschetz principle in general) and hence $T\left(\operatorname{Br}\left(X_{C}\right)\right)=0$ (see [Gro68, II, Corollaire 3.4]). As $T(\operatorname{Br}(X))$ is torsion-free, a norm argument now implies that $T(\operatorname{Br}(X))=0$. It follows that $E \otimes_{\mathbf{Z}} \widehat{\mathbf{Z}}=0$, hence that $E=0$.

2.3.2. Zero-cycles. On a smooth and proper variety of pure dimension $d$ over $R$, one may consider the real integral Hodge conjecture for zero-cycles in the sense of Definition 2.3 since $H^{q}\left(X, \Omega_{X}^{p}\right)=0$ for $p>d$ or $q>d$. We show that it always holds.

Proposition 2.10. Any smooth and proper variety of pure dimension d over a real closed field satisfies the real integral Hodge conjecture for codimension d cycles.

Proof. Pick a point $x_{i}$ in each semi-algebraic connected component of $X(R)$ and a point $y_{j}$ in each semi-algebraic connected component of $X(C)$. By Theorem 1.18 and the next lemma, the classes $\operatorname{cl}\left(x_{i}\right)$ and $\operatorname{cl}\left(N_{C / R}\left(y_{j}\right)\right)$ generate $H_{G}^{2 d}(X(C), \mathbf{Z}(d))_{0}$.

Lemma 2.11. Let $X$ be a smooth and proper variety of pure dimension $d$ over a real closed field $R$.

(i) Sending a connected component of $X_{C}$ to the cycle class of one of its closed

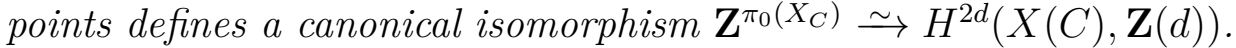

(ii) The norm map $H^{2 d}(X(C), \mathbf{Z}(d)) \rightarrow H_{G}^{2 d}(X(C), \mathbf{Z}(d))$ from (1.7) takes its values in the subgroup $H_{G}^{2 d}(X(C), \mathbf{Z}(d))_{0}$ and fits into an exact sequence

$$
H^{2 d}(X(C), \mathbf{Z}(d)) \longrightarrow H_{G}^{2 d}(X(C), \mathbf{Z}(d))_{0} \longrightarrow H^{d}(X(R), \mathbf{Z} / 2 \mathbf{Z}) \longrightarrow 0
$$

where the map on the right is the map (1.58) for $k=d$ and where the map on the left is injective if $X$ is geometrically connected.

Proof. Assertion (i) is a part of Poincaré duality and follows from Mil80, Chapter VI, Theorem 11.1 (a)] by comparison with $\ell$-adic cohomology for every $\ell$. Alternatively, although we do not do it here, it could be deduced from the statement of Poincaré duality given in Proposition 1.1.

The composition of the norm map which appears in (ii) with the natural map $H_{G}^{2 d}(X(C), \mathbf{Z}(d)) \rightarrow H^{2 d}(X(C), \mathbf{Z}(d))$ coincides, if $X$ is geometrically irreducible, with multiplication by 2 on the torsion-free group $H^{2 d}(X(C), \mathbf{Z}(d))=\mathbf{Z}$. It is therefore injective in this case. The rest of the assertion follows from the commutative 
diagram

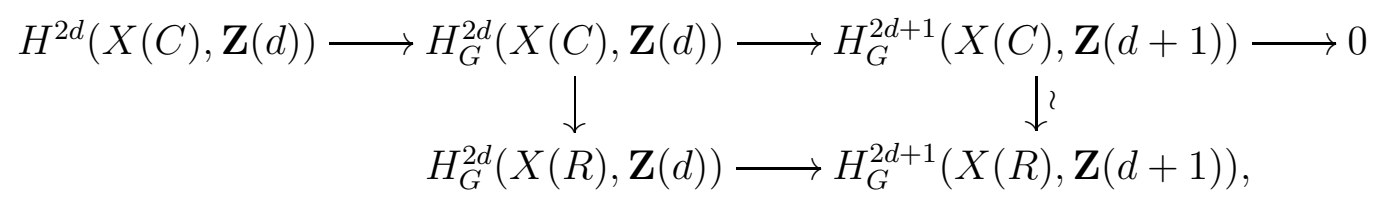

whose first row is exact and comes from (1.7). Indeed, the vertical map on the right is an isomorphism (see \$1.1.3) and the bottom horizontal map is compatible with the decompositions (1.30) (see $\$ 1.2 .3$ and $\$ 1.2 .4$ ).

Remark 2.12. Thanks to Proposition 2.10, we can now extend [Kuc96, Theorem 2.1] to the following purely cohomological statement, as promised in Remark 2.7 (ii):

Let $X$ be a smooth and proper variety of pure dimension $d$ over a real closed field $R$. Let $k$ be an integer. Let $\alpha_{k} \in H^{k}(X(R), \mathbf{Z} / 2 \mathbf{Z})$ and $\beta_{d-k} \in H^{d-k}(X(R), \mathbf{Z} / 2 \mathbf{Z})$. If $\alpha_{k}$ and $\beta_{d-k}$ can be lifted, by the corresponding maps (1.58), to $\alpha \in H_{G}^{2 k}(X(C), \mathbf{Z}(k))_{0}$ and $\beta \in H_{G}^{2 d-2 k}(X(C), \mathbf{Z}(d-k))_{0}$ such that the image of $\beta$ in $H^{2 d-2 k}(X(C), \mathbf{Z}(d-k))$ vanishes (as is the case if $\beta=\operatorname{cl}(y)$ for a cycle $y$ algebraically equivalent to 0 ), then $\operatorname{deg}\left(\alpha_{k} \smile \beta_{d-k}\right)=0$, where $\operatorname{deg}: H^{d}(X(R), \mathbf{Z} / 2 \mathbf{Z}) \rightarrow \mathbf{Z} / 2 \mathbf{Z}$ denotes the total degree.

Indeed, letting $\gamma=\alpha \smile \beta$, we have $\gamma \in H_{G}^{2 d}(X(C), \mathbf{Z}(d))_{0}$ and $\gamma_{d}=\alpha_{k} \smile \beta_{d-k}$ by \$1.6.4. By Proposition 2.10, there exists a zero-cycle $z$ such that $\gamma=\operatorname{cl}(z)$. It must have degree 0 since the image of $\gamma$ in $H^{2 d}(X(C), \mathbf{Z}(d))$ vanishes. Hence $\operatorname{deg}\left(\gamma_{d}\right)=0$.

2.3.3. Birational invariance. The integral Hodge conjecture for codimension $k$ cycles on a smooth and proper complex algebraic variety of pure dimension $d$ is a birational invariant when $k=2$ or $k=d-1$ (see [Voi07, Lemma 15]). The real integral Hodge conjecture enjoys the same birational invariance property.

Proposition 2.13. Let $R$ be a real closed field. Let $X$ denote a smooth and proper variety of pure dimension d over $R$. Let $k \in\{2, d-1\}$. If $R=\mathbf{R}$, the group

$$
\operatorname{Coker}\left(\mathrm{CH}^{k}(X) \rightarrow \operatorname{Hdg}_{G}^{2 k}(X(\mathbf{C}), \mathbf{Z}(k))_{0}\right)
$$

is a birational invariant of $X$. If $k=d-1$, so is the group

$$
\operatorname{Coker}\left(\mathrm{CH}^{k}(X) \rightarrow H_{G}^{2 k}(X(C), \mathbf{Z}(k))_{0}\right),
$$

for any $R$.

Proof. By Hironaka's theorem [Hir64, §5], it suffices to show that these groups are invariant by any blowing-up with smooth centre. This, in turn, follows from the next lemma and from the validity of the real integral Hodge conjecture for divisors and for zero-cycles (Propositions 2.8, 2.9, and 2.10) on the centre of the blowing-up. 
Lemma 2.14. Let $\pi: X^{\prime} \rightarrow X$ denote the blowing-up of a smooth irreducible closed subvariety $Z \subset X$ of codimension $r$. Let $\iota: E \hookrightarrow X^{\prime}$ denote the inclusion of the exceptional divisor and $\tau: E \rightarrow Z$ the projection. Let $k$ be an integer. Let $\xi$ denote the image, by $\mathrm{cl}: \operatorname{Pic}(E) \rightarrow H_{G}^{2}\left(E(C), \mathbf{Z}(1)\right.$ ), of the class of $\mathscr{O}_{E}(1)$ (the tautological line bundle of the projective bundle $\tau)$. There is a natural isomorphism

$$
H_{G}^{2 k}(X(C), \mathbf{Z}(k))_{0} \oplus \bigoplus_{i=k-r+1}^{k-1} H_{G}^{2 i}(Z(C), \mathbf{Z}(i))_{0} \rightarrow H_{G}^{2 k}\left(X^{\prime}(C), \mathbf{Z}(k)\right)_{0}
$$

and, if $R=\mathbf{R}$, a natural isomorphism

$$
\operatorname{Hdg}_{G}^{2 k}(X(C), \mathbf{Z}(k))_{0} \oplus \bigoplus_{i=k-r+1}^{k-1} \operatorname{Hdg}_{G}^{2 i}(Z(C), \mathbf{Z}(i))_{0} \rightarrow \operatorname{Hdg}_{G}^{2 k}\left(X^{\prime}(C), \mathbf{Z}(k)\right)_{0},
$$

both given by $\alpha \oplus \oplus\left(\beta_{i}\right)_{i} \mapsto \pi^{*} \alpha+\sum_{i} \iota_{*}\left(\xi^{k-i-1} \smile \tau^{*} \beta_{i}\right)$.

Proof. As is well known, the above formula defines an isomorphism of abelian groups

$$
H_{G}^{2 k}(X(C), \mathbf{Z}(k)) \oplus \bigoplus_{i=k-r+1}^{k-1} H_{G}^{2 i}(Z(C), \mathbf{Z}(i)) \rightarrow H_{G}^{2 k}\left(X^{\prime}(C), \mathbf{Z}(k)\right)
$$

(see [Kat73, Théorème 2.2, Théorème 1.2]). When $R=\mathbf{R}$, this isomorphism respects the Hodge decompositions. It only remains to be checked that for any $\alpha \oplus \oplus\left(\beta_{i}\right)_{i}$ in the left-hand side of (2.4), if one sets $\gamma=\pi^{*} \alpha+\sum_{i} \iota_{*}\left(\xi^{k-i-1} \smile \tau^{*} \beta_{i}\right)$, then $\gamma \in$ $H_{G}^{2 k}\left(X^{\prime}(C), \mathbf{Z}(k)\right)_{0}$ if and only if $\alpha \in H_{G}^{2 k}(X(C), \mathbf{Z}(k))_{0}$ and $\beta_{i} \in H_{G}^{2 i}(Z(C), \mathbf{Z}(i))_{0}$ for every $i$. As cup products, push-forwards and pull-backs preserve the condition of Definition 1.19 (see \$1.6.4 and Theorem 1.21), the converse implication is clear. For the direct implication, let

$$
\delta=\sum_{i=k-r+1}^{k-1} \xi^{k-i} \smile \tau^{*} \beta_{i} \in H_{G}^{2 k}(E(C), \mathbf{Z}(k))
$$

and let us assume that $\gamma \in H_{G}^{2 k}\left(X^{\prime}(C), \mathbf{Z}(k)\right)_{0}$. As $\alpha=\pi_{*} \gamma$, Theorem 1.21 shows that $\alpha \in H_{G}^{2 k}(X(C), \mathbf{Z}(k))_{0}$. As $\delta=\iota^{*}\left(\gamma-\pi^{*} \alpha\right)$, it follows that $\delta \in H_{G}^{2 k}(E(C), \mathbf{Z}(k))_{0}$. To prove that $\beta_{i} \in H_{G}^{2 i}(Z(C), \mathbf{Z}(i))_{0}$ for all $i$, we now argue by induction on $i$. Assuming that $i_{0} \in\{k-r+1, \ldots, k-1\}$ is such that $\beta_{i} \in H_{G}^{2 i}(Z(C), \mathbf{Z}(i))_{0}$ for all $i<i_{0}$, let us check that $\beta_{i_{0}} \in H_{G}^{2 i_{0}}\left(Z(C), \mathbf{Z}\left(i_{0}\right)\right)_{0}$. By the projection formula (1.23), we have

$$
\tau_{*}\left(\xi^{i_{0}-k+r-1} \smile \delta\right)=\beta_{i_{0}}+\sum_{i=k-r+1}^{i_{0}-1} \tau_{*} \xi^{i_{0}-i+r-1} \smile \beta_{i}
$$

since $\tau_{*} \xi^{m}=0$ for $m<r-1$ and $\tau_{*} \xi^{r-1}=1 \in H_{G}^{0}(Z(C), \mathbf{Z})$. By the compatibility of Definition 1.19 with push-forwards and cup products (see \$1.6.4 and Theorem 1.21), the left-hand side of (2.5) belongs to $H_{G}^{2 i_{0}}\left(Z(C), \mathbf{Z}\left(i_{0}\right)\right)_{0}$. By our assumption on $i_{0}$, so does the second term of the right-hand side; hence $\beta_{i_{0}} \in H_{G}^{2 i_{0}}\left(Z(C), \mathbf{Z}\left(i_{0}\right)\right)_{0}$. 
2.3.4. Projective spaces. We finally consider the case of projective spaces.

Proposition 2.15. For all integers $0 \leq k \leq d$, the real integral Hodge conjecture holds for codimension $k$ cycles on $\mathbf{P}_{R}^{d}$.

Proof. By Ful98, Theorem $3.3(\mathrm{~b})]$, the Chow group $\mathrm{CH}^{d}\left(\mathbf{P}_{R}^{d} \times \mathbf{P}_{R}^{d}\right)$ is generated by the classes of $\mathbf{P}_{R}^{i} \times \mathbf{P}_{R}^{d-i}$ for $0 \leq i \leq d$. The class of the diagonal $\Delta_{\mathbf{P}_{R}^{d}}$ may then be determined by computing the intersection numbers $\left[\Delta_{\mathbf{P}_{R}^{d}}\right] \cdot\left[\mathbf{P}_{R}^{i} \times \mathbf{P}_{R}^{d-i}\right]=1$ :

$$
\left[\Delta_{\mathbf{P}_{R}^{d}}\right]=\sum_{i=0}^{d}\left[\mathbf{P}_{R}^{i} \times \mathbf{P}_{R}^{d-i}\right] \in \mathrm{CH}^{d}\left(\mathbf{P}_{R}^{d} \times \mathbf{P}_{R}^{d}\right)
$$

Let $\alpha \in H_{G}^{2 k}\left(\mathbf{P}^{d}(C), \mathbf{Z}(k)\right)_{0}$. We let the correspondence (2.6) act on $\alpha$. For $i<k$, one has $\left[\mathbf{P}_{R}^{i} \times \mathbf{P}_{R}^{d-i}\right]_{*} \alpha=0$. Indeed, $H_{G}^{2 k}\left(\mathbf{P}^{i}(C), \mathbf{Z}(k)\right) \rightarrow H_{G}^{2 k}\left(\mathbf{P}^{i}(R), \mathbf{Z}(k)\right)$ is injective by $\$ 1.1 .3$, so that $H_{G}^{2 k}\left(\mathbf{P}^{i}(C), \mathbf{Z}(k)\right)_{0}=0$. For $i>k$, one also has $\left[\mathbf{P}_{R}^{i} \times \mathbf{P}_{R}^{d-i}\right]_{*} \alpha=0$ in view of the vanishing of $H_{G}^{2 k-2 i}\left(\mathbf{P}^{d-i}(C), \mathbf{Z}(k-i)\right)$ in this range. We deduce:

$$
\alpha=\left[\Delta_{\mathbf{P}_{R}^{d}}\right]_{*} \alpha=\left[\mathbf{P}_{R}^{k} \times \mathbf{P}_{R}^{d-k}\right]_{*} \alpha .
$$

The latter class is algebraic because the whole group $H_{G}^{2 k}\left(\mathbf{P}^{k}(R), \mathbf{Z}(k)\right)_{0}$ consists of algebraic classes by Proposition 2.10.

2.4. Main question. The results of Voisin [Voi06, Voi13 on the integral Hodge conjecture for complex varieties (discussed in \$2.1) and the good basic properties of the real integral Hodge conjecture (see \$2.3) lead us to raise the following question:

Question 2.16. Let $X$ be a smooth, proper and geometrically irreducible variety over $\mathbf{R}$. If the underlying complex variety $X_{\mathbf{C}}$ is a uniruled threefold, a Calabi-Yau threefold, or a rationally connected variety, does $X$ satisfy the real integral Hodge conjecture for 1-cycles?

In \$3 and \$5 below, we explore the meaning and implications of a positive answer to this question. In [BW18], we shall provide positive answers for various classes of uniruled threefolds (and, in higher dimensions, for iterated conic bundles over these threefolds, or over arbitrary surfaces). As far as we know, the answer to Question 2.16 may be in the affirmative in full generality.

For Calabi-Yau threefolds and for rationally connected varieties, one might wish to extend Question 2.16 to arbitrary real closed fields, using Definition 2.3. However, we shall give in BW18] various examples which show that the resulting question would have a negative answer, at least for rationally connected threefolds with a rational point and for (simply connected) Calabi-Yau threefolds with no rational point. (It is still conceivable that over an arbitrary real closed field, rationally connected varieties with no rational point may satisfy the real integral Hodge conjecture for 1-cycles.) 


\section{CyCles OF Dimension 1}

The goal of this section is to investigate the relationship between the real integral Hodge conjecture for 1-cycles, on the one hand, and the complex integral Hodge conjecture for 1-cycles, the study of the Borel-Haefliger cycle class map, and the question of the existence of curves of even geometric genus in a given variety, on the other hand. For the whole of \$3, we fix a real closed field $R$ and a smooth, proper and geometrically irreducible variety $X$ over $R$, of dimension $d$.

3.1. Summary. Before examining the integral Hodge conjecture for 1-cycles, we must study the target $H_{G}^{2 d-2}(X(C), \mathbf{Z}(d-1))_{0}$ of the equivariant cycle class map.

A key role will be played by two homomorphisms

$$
\psi: H_{G}^{2 d-2}(X(C), \mathbf{Z}(d-1))_{0} \rightarrow \mathscr{M} \quad \text { and } \quad \psi^{\prime}: \operatorname{Pic}\left(X_{C}\right)^{G}\left[2^{\infty}\right] \rightarrow \mathscr{M}^{*},
$$

where $\mathscr{M}$ is a 2 -torsion abelian group and $\mathscr{M}^{*}=\operatorname{Hom}(\mathscr{M}, \mathbf{Z} / 2 \mathbf{Z})$. Their definitions, given in \$3.2, are of a purely topological nature. Theorem 3.3, whose proof relies on the self-dual long exact sequence of Theorem[1.12, asserts that the images of $\psi$ and $\psi^{\prime}$ are exact orthogonal complements under the natural pairing $\mathscr{M} \times \mathscr{M}^{*} \rightarrow \mathbf{Z} / 2 \mathbf{Z}$; in particular, the map $\psi$ is surjective if $\operatorname{Pic}\left(X_{C}\right)[2]=0$. We show, in \$3.5, that under the same assumption, the kernel of $\psi$ coincides with the image of the norm map

$$
N_{C / R}: H^{2 d-2}(X(C), \mathbf{Z}(d-1)) \rightarrow H_{G}^{2 d-2}(X(C), \mathbf{Z}(d-1)) .
$$

Thus, we obtain, in this case, a complete description of $H_{G}^{2 d-2}(X(C), \mathbf{Z}(d-1))_{0}$.

To define $\psi$ and $\psi^{\prime}$ and to prove these results, we have to distinguish between the two cases $X(R)=\varnothing$ and $X(R) \neq \varnothing$. The definitions and arguments, in these two cases, are entirely distinct, but run parallel.

In \$3.3, we study the map $\varphi=\psi \circ \mathrm{cl}: \mathrm{CH}_{1}(X) \rightarrow \mathscr{M}$. It turns out that $\varphi$ coincides with the Borel-Haefliger cycle class map when $X(R) \neq \varnothing$, and that when $X(R)=\varnothing$, this map computes the geometric genus modulo 2 of the curves lying on $X$.

We gather several consequences of these results in \$3.6. In the case of surfaces $X$ with $H^{2}\left(X, \mathscr{O}_{X}\right)=0$, we obtain a statement of independent interest on the image of the Borel-Haefliger cycle class map and on the existence of curves of even geometric genus (Corollary 3.17), which generalises, in particular, the work of Mangolte and van Hamel [MvH98] on Enriques surfaces. In the case of simply connected CalabiYau or rationally connected threefolds, we obtain, by combining these results with the work of Voisin Voi06, an equivalence between the real integral Hodge conjecture and the surjectivity of the Borel-Haefliger cycle class map and the existence of a curve of even geometric genus (Corollary 3.23 ).

3.2. The map $\psi$ and its image. We first define the 2-torsion abelian group $\mathscr{M}$ and the homomorphisms $\psi$ and $\psi^{\prime}$. Once $\mathscr{M}$ is defined, we set $\mathscr{M}^{*}=\operatorname{Hom}(\mathscr{M}, \mathbf{Z} / 2 \mathbf{Z})$. 
Definition 3.1. If $X(R)=\varnothing$, we let $\mathscr{M}=\mathbf{Z} / 2 \mathbf{Z}$, we define

$$
\psi: H_{G}^{2 d-2}(X(C), \mathbf{Z}(d-1))_{0} \rightarrow \mathscr{M}
$$

by $\psi(x)=x \smile \omega^{2}$ for any $x$ (see $\$ 1.1 .2$ for the definition of $\omega^{2} \in H_{G}^{2}(X(C), \mathbf{Z}(2))$ ), which makes sense as $H_{G}^{2 d}(X(C), \mathbf{Z}(d+1))=\mathbf{Z} / 2 \mathbf{Z}$ in view of Proposition 1.10 and Remark 1.11 (ii), and we define

$$
\psi^{\prime}: \operatorname{Pic}\left(X_{C}\right)^{G}\left[2^{\infty}\right] \rightarrow \mathscr{M}^{*}
$$

as the restriction to $\operatorname{Pic}\left(X_{C}\right)^{G}\left[2^{\infty}\right]$ of the map $\operatorname{Pic}\left(X_{C}\right)^{G} \rightarrow \operatorname{Br}(R)=\mathbf{Z} / 2 \mathbf{Z}=\mathscr{M}^{*}$ which measures the obstruction to representing a $G$-invariant divisor class on $X_{C}$ by a G-invariant divisor (see [Gro68, III, §5.4]).

Definition 3.2. If $X(R) \neq \varnothing$, we let $\mathscr{M}=H^{d-1}(X(R), \mathbf{Z} / 2 \mathbf{Z})$, we define

$$
\psi: H_{G}^{2 d-2}(X(C), \mathbf{Z}(d-1))_{0} \rightarrow \mathscr{M}
$$

to be the map (1.58), and, noting that $\mathscr{M}^{*}=H^{1}(X(R), \mathbf{Z} / 2 \mathbf{Z})$ (see (1.16)) and that $\operatorname{Pic}\left(X_{C}\right)^{G}=\operatorname{Pic}(X)$ as $X(R) \neq \varnothing($ see $[$ BLR90, 8.1/4]), we define

$$
\psi^{\prime}: \operatorname{Pic}\left(X_{C}\right)^{G}\left[2^{\infty}\right] \rightarrow \mathscr{M}^{*}
$$

as the restriction to $\operatorname{Pic}\left(X_{C}\right)^{G}\left[2^{\infty}\right]=\operatorname{Pic}(X)\left[2^{\infty}\right]$ of the Borel-Haefliger cycle class $\operatorname{map} \operatorname{Pic}(X) \rightarrow H^{1}(X(R), \mathbf{Z} / 2 \mathbf{Z})($ see $(1.56))$.

Theorem 3.3. For any smooth, proper and geometrically irreducible variety $X$, of dimension $d$, over a real closed field $R$, the images of $\psi$ and of $\psi^{\prime}$ are exact orthogonal complements under the natural perfect pairing $\mathscr{M} \times \mathscr{M}^{*} \rightarrow \mathbf{Z} / 2 \mathbf{Z}$.

Proof. We first prove the theorem under the assumption that $X(R)=\varnothing$. In this case, we have to show that $\psi$ is surjective if and only if $\psi^{\prime}=0$. Let us consider the commutative square

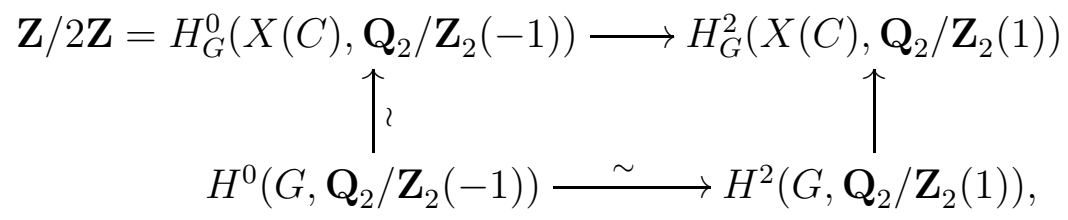

in which the horizontal maps are the cup product with $\omega^{2}$ and the vertical maps come from the Hochschild-Serre spectral sequence (1.4). Applying (1.1) and (1.2), we see that the bottom horizontal map is an isomorphism. On the other hand, by Proposition 1.10 and Remark 1.11 (ii), the top horizontal map composed with the inclusion $H_{G}^{2}\left(X(C), \mathbf{Q}_{2} / \mathbf{Z}_{2}(1)\right) \subseteq H_{G}^{2}(X(C), \mathbf{Q} / \mathbf{Z}(1))$ is dual to $\psi$. Hence $\psi$ is surjective if and only if the vertical map on the right is injective. By (1.4), this amounts to the surjectivity of the natural map $H_{G}^{1}\left(X(C), \mathbf{Q}_{2} / \mathbf{Z}_{2}(1)\right) \rightarrow H^{1}\left(X(C), \mathbf{Q}_{2} / \mathbf{Z}_{2}(1)\right)^{G}$, or equivalently, of $H_{\text {êt }}^{1}\left(X, \mathbf{Q}_{2} / \mathbf{Z}_{2}(1)\right) \rightarrow H_{\text {êt }}^{1}\left(X_{C}, \mathbf{Q}_{2} / \mathbf{Z}_{2}(1)\right)^{G}$. The Kummer exact sequence in 
étale cohomology identifies the latter map with $\operatorname{Pic}(X)\left[2^{\infty}\right] \rightarrow \operatorname{Pic}\left(X_{C}\right)^{G}\left[2^{\infty}\right]$, which is surjective if and only if $\psi^{\prime}=0$.

We henceforth assume that $X(R) \neq \varnothing$. Let us state two lemmas.

As $H^{0}(G, \mathbf{Q} / \mathbf{Z}(1))=\mathbf{Z} / 2 \mathbf{Z}$ and $H^{1}(G, \mathbf{Q} / \mathbf{Z}(1))=0$, the spectral sequence (1.3) induces a canonical isomorphism $\mathscr{M}^{*}=H^{1}(X(R), \mathbf{Z} / 2 \mathbf{Z}) \stackrel{\sim}{\rightarrow} H_{G}^{1}(X(R), \mathbf{Q} / \mathbf{Z}(1))$.

Lemma 3.4. Through this identification of $\mathscr{M}^{*}$ with $H_{G}^{1}(X(R), \mathbf{Q} / \mathbf{Z}(1))$, the image of $\psi^{\prime}$ is the image of the restriction map $H_{G}^{1}(X(C), \mathbf{Q} / \mathbf{Z}(1)) \rightarrow H_{G}^{1}(X(R), \mathbf{Q} / \mathbf{Z}(1))$.

Proof. The boundary maps of the exact sequence $0 \rightarrow \mathbf{Z}(1) \rightarrow \mathbf{Q}(1) \rightarrow \mathbf{Q} / \mathbf{Z}(1) \rightarrow 0$ and the isomorphism $\operatorname{Pic}(X)_{\text {tors }}=H_{\text {ét }}^{1}(X, \mathbf{Q} / \mathbf{Z}(1))=H_{G}^{1}(X(C), \mathbf{Q} / \mathbf{Z}(1))$ stemming from the Kummer sequence fit into a diagram

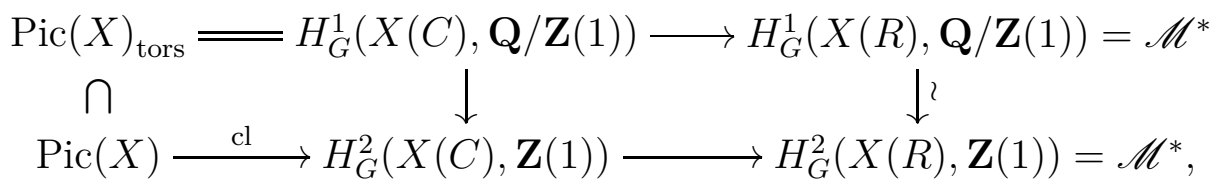

where we identify $H_{G}^{2}(X(R), \mathbf{Z}(1))$ with $\mathscr{M}^{*}$ by means of (1.31). In terms of these identifications, the rightmost vertical map of (3.1) is the identity map of $\mathscr{M}^{*}$, as follows from the commutative square

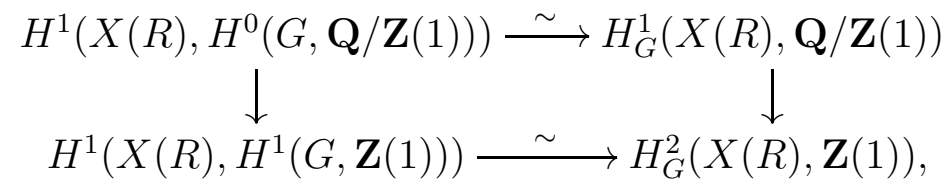

whose horizontal maps are the edge homomorphisms of the spectral sequences (1.3). According to [CT93, (3.8) and §3.3.1], the left square of (3.1) commutes after tensoring with $\mathbf{Z}_{\ell}$ for every prime $\ell$; therefore (3.1) is a commutative diagram. By Theorem 1.18, the image of $\operatorname{Pic}(X)\left[2^{\infty}\right]$ in $\mathscr{M}^{*}$, via (3.1), is the image of $\psi$ $($ see $(1.58))$. It is also the image of $\operatorname{Pic}(X)_{\text {tors }}$ since $2 \mathscr{M}^{*}=0$. The lemma follows.

Lemma 3.5. The restriction map induces an isomorphism

$$
H_{G}^{2 d-2}(X(C), \mathbf{Z}(d-1))_{0} \stackrel{\sim}{\longrightarrow} H_{G}^{2 d-2}(X(C) \backslash X(R), \mathbf{Z}(d-1)) .
$$

Proof. We recall that a canonical retraction of the forgetful map

$$
H_{G, X(R)}^{2 d-2}(X(C), \mathbf{Z}(d-1)) \rightarrow H_{G}^{2 d-2}(X(C), \mathbf{Z}(d-1))
$$

was constructed in \$1.3.2. By Proposition 1.7, the lemma will follow if we check that the kernel of this retraction is $H_{G}^{2 d-2}(X(C), \mathbf{Z}(d-1))_{0}$. Let us take up, from \$1.3.1, the notation $H^{p}=H^{p}(X(R), \mathbf{Z} / 2 \mathbf{Z})$. Let $\alpha \in H_{G}^{2 d-2}(X(C), \mathbf{Z}(d-1))$. As in \$1.6.3, we denote by $\alpha_{p} \in H^{p}$ the image of $\alpha$ by (1.57) for $p \equiv d-1 \bmod 2$. Unravelling the definition of the canonical retraction of (3.3) constructed in \$1.3.2, while taking \$1.2.3 
into account, shows that $\alpha$ belongs to its kernel if and only if the component in $H^{p}$ of the class

$$
\sum_{\substack{0 \leq i \leq d-3 \\ i \equiv d-1 \bmod 2}}\left(\alpha_{i}+\beta_{\mathbf{Z}}\left(\alpha_{i}\right)\right) \gamma^{-1} \in H^{0} \oplus \cdots \oplus H^{d-2}
$$

is trivial for each $p \equiv d-1 \bmod 2$ such that $p \leq d-3$. Here, the product takes place in the ring $H^{0} \oplus \cdots \oplus H^{d-2}$ and $\beta_{\mathbf{Z}}: H^{i} \rightarrow H^{i+1}$ denotes the ordinary Bockstein homomorphism (see \$1.2.3). On the other hand, we have $\alpha \in H_{G}^{2 d-2}(X(C), \mathbf{Z}(d-1))_{0}$ if and only if $\alpha_{p}=0$ for each $p \leq d-3$ such that $p \equiv d-1 \bmod 2$. To see that these two conditions are equivalent, we note that if $\alpha_{p} \neq 0$ for some $p \equiv d-1 \bmod 2$ and if $p$ denotes the smallest such integer, then the component in $H^{p}$ of (3.4) is $\alpha_{p}$.

Let us resume the proof of Theorem 3.3 under the assumption that $X(R) \neq \varnothing$. The map $u_{2}$ appearing in (1.46) can be inserted into a diagram

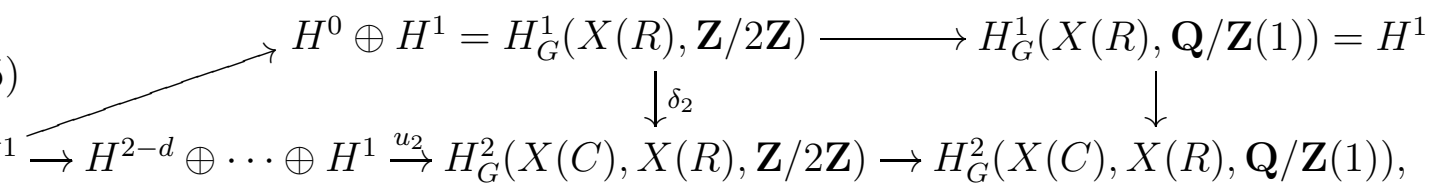

in which the leftmost arrows are the obvious inclusions, the vertical maps are the connecting homomorphisms of the localisation exact sequences, and the canonical isomorphisms of the first row are the decomposition (1.26) and the identification used in the statement of Lemma 3.4. A glance at the description of $u_{2}$ given after (1.46) shows that this diagram commutes. In addition, the map from the bottom left $H^{1}$ to the top right $H^{1}$ is the identity map. It follows, in view of Lemma 3.4, that the kernel of the composition $\theta^{\prime}: H^{1} \rightarrow H_{G}^{2}(X(C), X(R), \mathbf{Q} / \mathbf{Z}(1))$ of all of the maps of the bottom row of (3.5) coincides with the image of $\psi^{\prime}$.

Now, let $\theta: H_{G}^{2 d-2}(X(C) \backslash X(R), \mathbf{Z}(d-1)) \rightarrow H^{d-1}$ denote the composition of the natural map $H_{G}^{2 d-2}(X(C) \backslash X(R), \mathbf{Z}(d-1)) \rightarrow H_{G}^{2 d-2}(X(C) \backslash X(R), \mathbf{Z} / 2 \mathbf{Z})$, of the $\operatorname{map} w_{2 d-2}: H_{G}^{2 d-2}(X(C) \backslash X(R), \mathbf{Z} / 2 \mathbf{Z}) \rightarrow H^{d-1} \oplus \cdots \oplus H^{2 d-2}$ appearing in (1.46), and of the projection map $H^{d-1} \oplus \cdots \oplus H^{2 d-2} \rightarrow H^{d-1}$. The description of $w_{2 d-2}$ given after (1.46) shows, in view of \$1.2.3, that $\psi$ coincides with the composition of $\theta$ with the isomorphism of Lemma 3.5. Hence $\psi$ and $\theta$ have the same image.

By Theorem 1.12, Proposition 1.10, and Remark 1.11 (ii), the Pontrjagin dual of $\theta$ is $\theta^{\prime}$. The kernel of $\theta^{\prime}$ and the image of $\theta$ are therefore exact orthogonal complements.

3.3. The effect of $\psi$ on cycle classes. We set $\varphi=\psi \circ \mathrm{cl}: \mathrm{CH}_{1}(X) \rightarrow \mathscr{M}$.

Theorem 3.6. Let $X$ be a smooth, proper and geometrically irreducible variety over a real closed field $R$. If $X(R) \neq \varnothing$, the map $\varphi$ is the Borel-Haefliger cycle class map. 
If $X(R)=\varnothing$, the map $\varphi$ is characterised by the property that

$$
\varphi(B)= \begin{cases}1 & \text { if } B \text { is geometrically irreducible and has even geometric genus, } \\ 0 & \text { otherwise }\end{cases}
$$

for any integral proper curve $B \subseteq X$.

Proof. When $X(R) \neq \varnothing$, this is Theorem 1.18 applied with $i=0$. Let us assume that $X(R)=\varnothing$. If $B$ is not geometrically integral, then, as a 1-cycle on $X$, it is the norm of a 1-cycle on $X_{C}$, so that $\operatorname{cl}(B)$ belongs to the image of the norm map

$$
H^{2 d-2}(X(C), \mathbf{Z}(d-1)) \rightarrow H_{G}^{2 d-2}(X(C), \mathbf{Z}(d-1)) .
$$

In view of the real-complex long exact sequence (1.7), it follows that $\operatorname{cl}(B) \smile \omega=0$, hence $\varphi(B)=0$. We may therefore assume that $B$ is geometrically irreducible. Let $B^{\prime}$ denote its normalisation and $\pi: B^{\prime} \rightarrow X$ the natural morphism. The pushforward map $\pi_{*}: H_{G}^{2}\left(B^{\prime}(C), \mathbf{Z}(2)\right) \rightarrow H_{G}^{2 d}(X(C), \mathbf{Z}(d+1))$ is an isomorphism since its Pontrjagin dual $\pi^{*}: H_{G}^{0}(X(C), \mathbf{Q} / \mathbf{Z}(1)) \rightarrow H_{G}^{0}\left(B^{\prime}(C), \mathbf{Q} / \mathbf{Z}(1)\right)$ is one (see (1.23) and Proposition [1.10). As a consequence, if $\psi_{B^{\prime}}: H_{G}^{0}\left(B^{\prime}(C), \mathbf{Z}\right) \rightarrow \mathbf{Z} / 2 \mathbf{Z}$ denotes the map associated with $B^{\prime}$ by Definition 3.1, the square

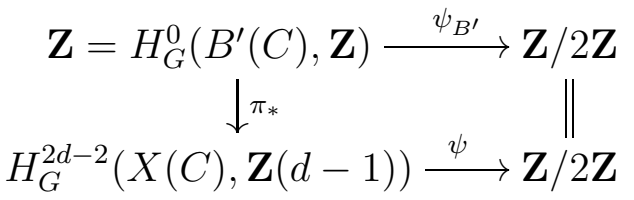

is commutative. By Theorem 3.3 and Lemma 3.7 below, the map $\psi_{B^{\prime}}$ is surjective if and only if $B^{\prime}$ has even genus. As the vertical arrow on the left maps 1 to $\operatorname{cl}(B)$, this completes the proof of Theorem 3.6.

Lemma 3.7 (Geyer). Let $B$ be a smooth, proper and geometrically integral curve over a real closed field $R$. If $B(R)=\varnothing$, the natural map $\operatorname{Pic}(B)\left[2^{\infty}\right] \rightarrow \operatorname{Pic}\left(B_{C}\right)^{G}\left[2^{\infty}\right]$ is surjective if and only if $B$ has even genus.

Proof. This follows from the work of Geyer (see Gey67, p. 91], extended to real closed fields in [Kne76b, §10]; see also [GH81, §2]). We sketch a complete argument for the reader's convenience. First, as $B(R)=\varnothing$ and $B$ is a curve, by a theorem of Witt [Wit34 extended by Pfister to real closed fields (see [Kne76a, Theorem 4.1] and the references therein), one can write -1 as a sum of two squares in $R(B)$. In other words, the natural map $\operatorname{Br}(R) \rightarrow \operatorname{Br}(B)$ vanishes. Hence, the exact sequence of low degree terms of the Hochschild-Serre spectral sequence takes the form

$$
0 \longrightarrow \operatorname{Pic}(B) \longrightarrow \operatorname{Pic}\left(B_{C}\right)^{G} \longrightarrow \operatorname{Br}(R) \longrightarrow 0 .
$$

Let $g$ denote the genus of $B$.

Sublemma 3.8. Let $L \in \operatorname{Pic}\left(B_{C}\right)^{G}$. If $\operatorname{deg}(L) \equiv g \bmod 2$, then $L \in \operatorname{Pic}(B)$. 
Proof. After tensoring $L$ with a large power of an ample line bundle, we may assume that $\operatorname{deg}(L)>2 g-2$. The Riemann-Roch theorem then implies that $\operatorname{dim}_{C} H^{0}\left(B_{C}, L\right)$ is odd. On the other hand, this integer kills the image of $L$ in $\operatorname{Br}(R)$ (see Lic69, Theorem 6] or [GH81, p. 160]).

The sublemma implies the lemma when $g$ is even. Suppose now $g$ is odd. By (3.6) there exists $L \in \operatorname{Pic}\left(B_{C}\right)^{G}$ with $L \notin \operatorname{Pic}(B)$. By the sublemma, its degree must be even. By adding to $L$ a suitable multiple of the class of a closed point of $B$, we may assume that $\operatorname{deg}(L)=0$. $\operatorname{As}_{\operatorname{Pic}^{0}}\left(B_{C}\right)$ is divisible, there exists $L_{0} \in \operatorname{Pic}^{0}\left(B_{C}\right)$ such that $L=2 L_{0}$. Then $L-N_{C / R}\left(L_{0}\right)$ is an element of $\operatorname{Pic}\left(B_{C}\right)^{G}[2]$ which does not belong to $\operatorname{Pic}(B)[2]$, thus completing the proof of the lemma.

3.4. Connection with the first intermediate index. According to Theorem 3.6 , the map $\varphi: \mathrm{CH}_{1}(X) \rightarrow \mathscr{M}$ is surjective if and only if

(i) the $\operatorname{map} \mathrm{cl}_{R}: \mathrm{CH}_{1}(X) \rightarrow H^{d-1}(X(R), \mathbf{Z} / 2 \mathbf{Z})$ is surjective, if $X(R) \neq \varnothing$;

(ii) $X$ contains a geometrically integral curve of even geometric genus, if $X(R)=\varnothing$.

The first condition is a classical one in real algebraic geometry. Since the second one is not, and will play a prominent role in what follows when $X(R)=\varnothing$, we devote this section to explaining a few general facts about it.

In the sequel, we shall encounter many examples of varieties, over the reals, which do not contain a curve of even geometric genus (see \$4 and [BW18, §9.2]). The following proposition illustrates the sharpness of such examples.

Proposition 3.9. Let $X$ be a smooth, proper and geometrically irreducible variety, of dimension $\geq 2$, over $R$ (or, more generally, over an infinite perfect field). Then $X$ contains a geometrically irreducible curve of odd geometric genus. If $X(R) \neq \varnothing$, then $X$ contains a geometrically irreducible curve of even geometric genus.

Proof. We first assume that $X$ is a surface. In this case, if $H$ denotes a very ample divisor on $X$, a general member of the linear system $|4 H|$ is a geometrically irreducible curve with odd geometric genus, as follows from the adjunction formula. If $X(R) \neq \varnothing$, let $\pi: X^{\prime} \rightarrow X$ denote the blowing-up of a point of $X(R)$, with exceptional divisor $E$, and let $H^{\prime}$ denote an ample divisor on $X^{\prime}$. The image, by $\pi$, of a general member of the linear system $\left|4 n H^{\prime}+E\right|$, for $n \gg 0$, is a geometrically irreducible curve with even geometric genus, according to the adjunction formula. The general case of Proposition 3.9 can be proved by applying these arguments to the desingularisation of an appropriate surface lying in $X$.

The existence of a curve of even geometric genus is conveniently expressed in terms of the intermediate indices of $X$, introduced by Kollár [Kol13, Definition 1] following [ELW15]. We recall that for any $i$, the $i$ th intermediate index $\operatorname{ind}_{i}(X)$ is, by 
definition, the gcd of the integers $\chi(X, E)$ when $E$ ranges over the coherent sheaves on $X$ supported on a closed subset of dimension $\leq i$. Clearly

$$
\operatorname{ind}_{d}(X)\left|\operatorname{ind}_{d-1}(X)\right| \cdots \mid \operatorname{ind}_{0}(X)
$$

and $\operatorname{ind}_{0}(X)$ is the gcd of the degrees of the closed points of $X$. The next statement, a consequence of standard results on Grothendieck groups of coherent sheaves (see [BS58, §8], [Ber71]), summarises some basic properties of these indices.

Proposition 3.10 ([Kol13, Proposition 4]). For any proper variety $X$ and any $i$, we have $\operatorname{ind}_{i}(X)=\operatorname{gcd} \chi\left(Z, \mathscr{O}_{Z}\right)=\operatorname{gcd} \chi\left(Z^{\prime}, \mathscr{O}_{Z^{\prime}}\right)$, where $Z$ ranges over the integral closed subvarieties of $X$ of dimension $\leq i$ and $Z^{\prime}$ denotes the normalisation of $Z$.

We can now relate $\operatorname{ind}_{1}(X)$ to curves of even genus. Note that if $X$ is a nonempty proper variety over $R$, then $\operatorname{ind}_{0}(X)=1$ if $X(R) \neq \varnothing$ and $\operatorname{ind}_{0}(X)=2$ otherwise.

Corollary 3.11. Let $X$ be a nonempty proper variety over $R$. If $X(R) \neq \varnothing$ or if $X$ contains a geometrically irreducible curve of even geometric genus, then $\operatorname{ind}_{1}(X)=1$. Otherwise $\operatorname{ind}_{1}(X)=2$.

As another consequence of Proposition [3.10, we note that for any proper and geometrically irreducible curve $B$ over $R$ such that $B(R)=\varnothing$, the geometric genus and the arithmetic genus of $B$ have the same parity (both are congruent to $1-\operatorname{ind}_{1}(B)$ modulo 2). Replacing the word "geometric" with "arithmetic" in the statements of Theorem 3.6 and Corollary 3.11 would therefore make no difference.

Finally, we recall how the parity of the genus behaves in a cover of curves.

Proposition 3.12. Let $f: E \rightarrow F$ be a finite morphism between smooth, proper and geometrically irreducible curves over $R$. Assume that $F(R)=\varnothing$ and that $F$ has even geometric genus. Then $E$ has even geometric genus if and only if $f$ has odd degree.

Proof. Apply the degree formula $\chi\left(E, \mathscr{O}_{E}\right) \equiv \operatorname{deg}(f) \chi\left(F, \mathscr{O}_{F}\right) \bmod 2$ (see [Hau13] or Kol13, Lemma $5(2)]$ ).

Remark 3.13. Let $X$ be a smooth and proper variety, defined over an arbitrary field. Let $i$ be an integer. Associating, with an integral dimension $i$ cycle on $X$, the Euler characteristic of its structure sheaf determines a morphism $\mathrm{CH}_{i}(X) \rightarrow \mathbf{Z} / \operatorname{ind}_{i-1}(X) \mathbf{Z}$ which factors through algebraic equivalence (see [Kol13, Proposition 14]). When the ground field is real closed and $i=1$, more is true: this morphism even factors through homological equivalence, according to Theorem 3.6.

We do not know whether this assertion remains valid for all values of $i$. For $i=1$, it does not extend to arbitrary fields, as the following example shows. Let $X$ denote the smooth projective quadric threefold over $\mathbf{C}((t))((u))((v))$ defined by the anisotropic quadratic form $\langle 1, t, u, t u, v\rangle$. Using the Hochschild-Serre spectral sequence, one checks that $H_{\text {ét }}^{4}\left(X, \mathbf{Z}_{2}(2)\right)$ is torsion-free. On the other hand, it follows from Kar90, 
Theorem 5.3 and Theorem 3.8] that $\mathrm{CH}_{1}(X)_{\text {tors }}$ has order 2 and is generated by a class whose image in $\mathbf{Z} / \operatorname{ind}_{0}(X) \mathbf{Z}=\mathbf{Z} / 2 \mathbf{Z}$ does not vanish.

\subsection{The cokernel of the norm map.}

Proposition 3.14. Let $X$ be a smooth, proper and geometrically irreducible variety, of dimension $d$, over a real closed field $R$. If $\operatorname{Pic}\left(X_{C}\right)[2]=0$, then $\psi$ fits into an exact sequence

$$
H^{2 d-2}(X(C), \mathbf{Z}(d-1)) \longrightarrow H_{G}^{2 d-2}(X(C), \mathbf{Z}(d-1))_{0} \stackrel{\psi}{\longrightarrow} \mathscr{M} \longrightarrow 0,
$$

where the first map is the norm map from (1.7).

It is part of the assertion of Proposition 3.14 that the norm map takes its values in the subgroup $H_{G}^{2 d-2}(X(C), \mathbf{Z}(d-1))_{0} \subseteq H_{G}^{2 d-2}(X(C), \mathbf{Z}(d-1))$.

Proof. The following lemma immediately implies Proposition 3.14 when $X(R)=\varnothing$.

Lemma 3.15. Under the assumptions of the proposition, the group $H^{2 d-1}(X(C), \mathbf{Z})$ is finite of odd order. Cup product with $\omega \in H_{G}^{1}(X(C), \mathbf{Z}(1))$ induces a surjection

$$
H_{G}^{2 d-2}(X(C), \mathbf{Z}(d-1)) \rightarrow H_{G}^{2 d-1}(X(C), \mathbf{Z}(d))[2]
$$

whose kernel is the image of the norm map and it induces an isomorphism

$$
H_{G}^{2 d-1}(X(C), \mathbf{Z}(d))[2] \stackrel{\sim}{\rightarrow} H_{G}^{2 d}(X(C), \mathbf{Z}(d+1)) .
$$

If $X(R) \neq \varnothing$, it also induces an isomorphism

$$
H_{G}^{2 d}(X(C), \mathbf{Z}(d+1)) \stackrel{\sim}{\longrightarrow} H_{G}^{2 d+1}(X(C), \mathbf{Z}(d+2)) .
$$

To deduce Proposition 3.14 from Lemma 3.15 when $X(R) \neq \varnothing$, we consider the commutative diagram

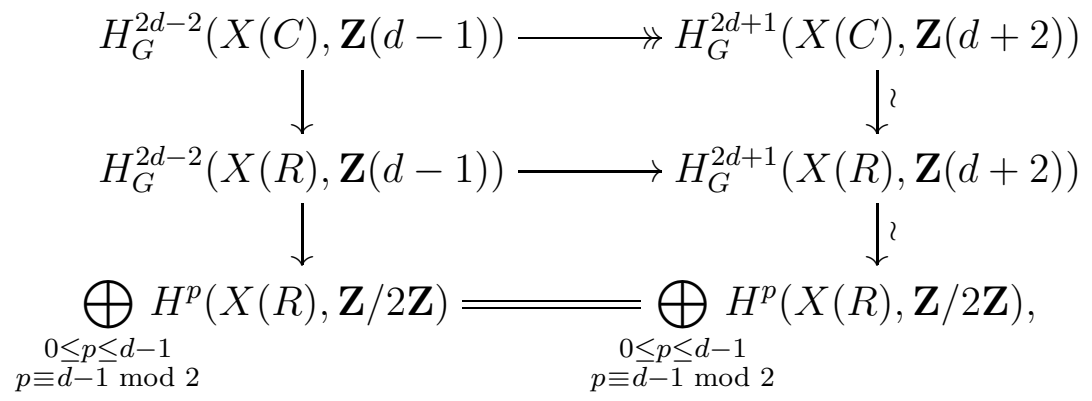

in which the horizontal arrows are the cup product with $\omega^{3}$ and the lower vertical maps are the maps (1.31). The commutativity of the lower square follows from \$1.2.3 and \$1.2.4, By Lemma 3.15, the top horizontal map is surjective and its kernel is the image of the norm map. The upper vertical map on the right is an isomorphism by $\$ 1.1 .3$. Hence the composition of the vertical maps on the left is surjective and its kernel is the image of the norm map. Proposition 3.14 is now established. 
Proof of Lemma [3.15. As Pic $\left(X_{C}\right)[2]=0$, the 2-torsion subgroup of $H_{\text {ét }}^{1}\left(X_{C}, \mathbf{Q}_{2} / \mathbf{Z}_{2}\right)$ is trivial. By Poincaré duality, the group $H_{\text {ét }}^{2 d-1}\left(X_{C}, \mathbf{Z}_{2}\right)=H^{2 d-1}(X(C), \mathbf{Z}) \otimes_{\mathbf{Z}} \mathbf{Z}_{2}$ is therefore 2-divisible. The group $H^{2 d-1}(X(C), \mathbf{Z})$ being finitely generated, it must then be finite of odd order. On the other hand, as $2 \omega=0$, the cohomology class $x \smile \omega$ is 2-torsion for any $x$, hence (3.8) is well-defined. It follows from these remarks and from the real-complex long exact sequence (1.7) that (3.8) is surjective, with kernel the image of the norm map.

The norm map $H^{2 d}(X(C), \mathbf{Z}(d)) \rightarrow H_{G}^{2 d}(X(C), \mathbf{Z}(d))$ is injective as its composition with the natural map $H_{G}^{2 d}(X(C), \mathbf{Z}(d)) \rightarrow H^{2 d}(X(C), \mathbf{Z}(d))$ is multiplication by 2 on the group $H^{2 d}(X(C), \mathbf{Z}(d))=\mathbf{Z}$ (see Lemma 2.11 (i)). Putting together this injectivity, the remarks that $H^{2 d-1}(X(C), \mathbf{Z})$ has odd order and that $2 \omega=0$, and the exact sequence (1.7), we deduce that (3.9) is an isomorphism.

If $X(R) \neq \varnothing$, the natural map $H_{G}^{2 d}(X(C), \mathbf{Z}(d)) \rightarrow H^{2 d}(X(C), \mathbf{Z}(d))$ is surjective (see Lemma 2.11 (i)). On the other hand, we have $H^{2 d+1}(X(C), \mathbf{Z}(d))=0$ as $X(C)$ has cohomological dimension $2 d$ (see [Del91, Chapter II, Lemma 9.1]). Hence the exact sequence (1.7) implies that (3.10) is an isomorphism in this case.

3.6. Wrapping up. We now combine the contents of $\$ \$ 3.23 .5$ and deduce various results on the Borel-Haefliger cycle class map for 1-cycles, on the existence of curves of even genus, and on the real integral Hodge conjecture for 1-cycles.

In accordance with common usage, for a smooth and proper variety $X$, we set $H_{1}(X(R), \mathbf{Z} / 2 \mathbf{Z})=H^{d-1}(X(R), \mathbf{Z} / 2 \mathbf{Z})$ and write $H_{1}^{\text {alg }}(X(R), \mathbf{Z} / 2 \mathbf{Z})=\mathrm{cl}_{R}\left(\mathrm{CH}_{1}(X)\right)$ and $H_{\text {alg }}^{1}(X(R), \mathbf{Z} / 2 \mathbf{Z})=\operatorname{cl}_{R}(\operatorname{Pic}(X))$, where $\mathrm{cl}_{R}$ denotes the Borel-Haefliger cycle class maps for curves or for divisors.

3.6.1. Nomenclature. We have seen in Theorem 3.6 that $\varphi=\psi \circ \mathrm{cl}: \mathrm{CH}_{1}(X) \rightarrow \mathscr{M}$ detects the genus modulo 2 if $X(R)=\varnothing$ and is the Borel-Haefliger cycle class map if $X(R) \neq \varnothing$. Let us analyse the various ways in which $\varphi$ can fail to be surjective. First, if $\psi$ is not surjective, we say that there is a topological obstruction to the surjectivity of $\varphi$. When $R=\mathbf{R}$, one can further factor $\varphi$ as

$$
\mathrm{CH}_{1}(X) \rightarrow \operatorname{Hdg}_{G}^{2 d-2}(X(C), \mathbf{Z}(d-1))_{0} \subseteq H_{G}^{2 d-2}(X(C), \mathbf{Z}(d-1))_{0} \stackrel{\psi}{\rightarrow} \mathscr{M} .
$$

If $R=\mathbf{R}$, we say that there is a Hodge-theoretic obstruction to the surjectivity of $\varphi$ if $\psi$ is surjective but its restriction to $\operatorname{Hdg}_{G}^{2 d-2}(X(C), \mathbf{Z}(d-1))_{0}$ is not. Finally, if $R=\mathbf{R}$ (resp., if $H^{2}\left(X, \mathscr{O}_{X}\right)=0$ ), we speak of a cycle-theoretic obstruction to the surjectivity of $\varphi$ if the Hodge-theoretic (resp., topological) obstruction vanishes but $\varphi$ still fails to be surjective.

There can be a topological obstruction only if $\operatorname{Pic}\left(X_{C}\right)[2] \neq 0$, by Theorem $[3.3$, there can be a Hodge-theoretic obstruction only if $H^{2}\left(X, \mathscr{O}_{X}\right) \neq 0$; and there can be a cycle-theoretic obstruction only if the real integral Hodge conjecture for 1-cycles fails for $X$. Examples illustrating all of these obstructions will be given in \$4. 
3.6.2. Varieties with $H^{2}\left(X, \mathscr{O}_{X}\right)=0$. Assume that $H^{2}\left(X, \mathscr{O}_{X}\right)=0$ ("no Hodgetheoretic obstruction") and that $X$ satisfies the real integral Hodge conjecture for 1-cycles ("no cycle-theoretic obstruction"). Then, by Theorem [3.3, the image of $\varphi=\psi \circ \mathrm{cl}: \operatorname{Pic}(X) \rightarrow \mathscr{M}$ is the orthogonal complement of the image of $\psi^{\prime}$ ("the topological obstruction controls the image of $\left.\varphi^{\prime \prime}\right)$. Combining this with Theorem 3.6 and Proposition 3.9, we have now established the following statement, in which $\mathrm{cl}_{R}$ denotes the two Borel-Haefliger cycle class maps $\operatorname{Pic}(X) \rightarrow H^{1}(X(R), \mathbf{Z} / 2 \mathbf{Z})$ and $\mathrm{CH}_{1}(X) \rightarrow H^{d-1}(X(R), \mathbf{Z} / 2 \mathbf{Z})$.

Theorem 3.16. Let $X$ be a smooth, proper and geometrically irreducible variety over a real closed field $R$. Assume that $\operatorname{dim}(X)>0$, that $H^{2}\left(X, \mathscr{O}_{X}\right)=0$ and that $X$ satisfies the real integral Hodge conjecture for 1-cycles.

(i) The subgroups $\mathrm{cl}_{R}\left(\mathrm{CH}_{1}(X)\right)$ and $\mathrm{cl}_{R}\left(\mathrm{Pic}(X)\left[2^{\infty}\right]\right)$ are exact orthogonal complements under the Poincaré duality pairing.

(ii) There exists a geometrically irreducible curve of even geometric genus in $X$ if and only if the natural map $\operatorname{Pic}(X)\left[2^{\infty}\right] \rightarrow \operatorname{Pic}\left(X_{C}\right)^{G}\left[2^{\infty}\right]$ is onto.

In particular, if $\operatorname{Pic}\left(X_{C}\right)[2]=0$, then $H_{1}^{\text {alg }}(X(R), \mathbf{Z} / 2 \mathbf{Z})=H_{1}(X(R), \mathbf{Z} / 2 \mathbf{Z})$ and $X$ contains a geometrically irreducible curve of even geometric genus.

According to Proposition 2.9, the hypotheses of Theorem 3.16] are met for surfaces of geometric genus zero.

Corollary 3.17. Let $X$ be a smooth, proper and geometrically irreducible surface over a real closed field $R$, such that $H^{2}\left(X, \mathscr{O}_{X}\right)=0$.

(i) The subgroups $\mathrm{cl}_{R}(\operatorname{Pic}(X))$ and $\mathrm{cl}_{R}\left(\operatorname{Pic}(X)\left[2^{\infty}\right]\right)$ of $H^{1}(X(R), \mathbf{Z} / 2 \mathbf{Z})$ are exact orthogonal complements under the Poincaré duality pairing.

(ii) There exists a geometrically irreducible curve of even geometric genus in $X$ if and only if the natural map $\operatorname{Pic}(X)\left[2^{\infty}\right] \rightarrow \operatorname{Pic}\left(X_{C}\right)^{G}\left[2^{\infty}\right]$ is onto.

In particular, if $\operatorname{Pic}\left(X_{C}\right)[2]=0$, then $H_{\mathrm{alg}}^{1}(X(R), \mathbf{Z} / 2 \mathbf{Z})=H^{1}(X(R), \mathbf{Z} / 2 \mathbf{Z})$ and $X$ contains a geometrically irreducible curve of even geometric genus.

Remarks 3.18. (i) At least when $R=\mathbf{R}$, the particular case of Corollary 3.17 (i) when $\operatorname{Pic}\left(X_{C}\right)[2]=0$ was known to Silhol [Sil89, Theorem III.3.4] for geometrically rational surfaces and to van Hamel vH00a, Chapter IV, Corollary 4.4 and Chapter III, Lemma 8.9] in general; see also [Man17, Théorème 3.7.18]. Corollary 3.17(ii), on the other hand, is new even when $\operatorname{Pic}\left(X_{C}\right)[2]=0$.

(ii) Assume that $R=\mathbf{R}$. According to [Kah87, Théorème 4], the map $\mathrm{cl}_{\mathbf{R}}$ sends the isomorphism class of a line bundle on $X$ to the first Stiefel-Whitney class of the line bundle it induces on $X(\mathbf{R})$. In particular, if $K_{X}$ denotes the canonical divisor class of $X$ and $w_{1}(X(\mathbf{R}))$ the first Stiefel-Whitney class of the tangent bundle of $X(\mathbf{R})$, then $\mathrm{cl}_{\mathbf{R}}\left(K_{X}\right)=w_{1}(X(\mathbf{R}))$. On the other hand, for an Enriques surface $X$, the group $\operatorname{Pic}(X)\left[2^{\infty}\right]$ has order 2 and is generated by $K_{X}$. Corollary 3.17 (i) 
therefore recovers the theorem of Mangolte and van Hamel [MvH98, Theorem 4.4] according to which if $X$ is an Enriques surface, the subgroup $H_{\text {alg }}^{1}(X(\mathbf{R}), \mathbf{Z} / 2 \mathbf{Z})$ is the orthogonal complement of $w_{1}\left(X(\mathbf{R})\right.$ ) (so that the equality $H_{\text {alg }}^{1}(X(\mathbf{R}), \mathbf{Z} / 2 \mathbf{Z})=$ $H^{1}(X(\mathbf{R}), \mathbf{Z} / 2 \mathbf{Z})$ holds if and only if every connected component of $X(\mathbf{R})$ is orientable). Corollary [3.17 (i) may be viewed as a generalisation of this result to all surfaces of geometric genus zero and all real closed fields.

(iii) Corollary 3.17 (i) was known to Kucharz when $R=\mathbf{R}$ and $X_{\mathbf{C}}$ is birationally ruled (see [Kuc01, Proposition 1.6]; note that $\mathrm{NS}(X)$ is torsion-free in this case, so that $\left.\operatorname{cl}_{\mathbf{R}}\left(\operatorname{Pic}(X)\left[2^{\infty}\right]\right)=\operatorname{cl}_{\mathbf{R}}\left(\operatorname{Pic}^{0}(X)\left[2^{\infty}\right]\right)=\operatorname{cl}_{\mathbf{R}}\left(\operatorname{Pic}^{0}(X)\right)\right)$.

(iv) Both assertions of Corollary 3.17 fail if we drop the assumption $H^{2}\left(X, \mathscr{O}_{X}\right)=0$ (see Example 4.5 and Example 4.6; these are $K 3$ surfaces).

Example 3.19. By Corollary 3.17 (ii), every Enriques surface over a real closed field contains a geometrically irreducible curve of even geometric genus. Indeed, if $X$ is such a surface, then $H^{2}\left(X, \mathscr{O}_{X}\right)=0$ and $\operatorname{Pic}\left(X_{C}\right)\left[2^{\infty}\right]$ is generated by the canonical class $K_{X_{C}}$, which comes, by pull-back, from the class $K_{X}$ in $\operatorname{Pic}(X)[2]$.

As a consequence of Corollary 3.17 (i), we obtain a lower bound on the size of the subgroup $H_{\text {alg }}^{1}(X(R), \mathbf{Z} / 2 \mathbf{Z}) \subseteq H^{1}(X(R), \mathbf{Z} / 2 \mathbf{Z})$, yielding a positive result concerning the question raised in [BT82, Remark 5.2].

Corollary 3.20. Let $X$ be a smooth, proper and geometrically irreducible surface over a real closed field $R$. Assume that $H^{2}\left(X, \mathscr{O}_{X}\right)=0$. Then

$$
\operatorname{dim}_{\mathbf{Z} / 2 \mathbf{Z}} H_{\mathrm{alg}}^{1}(X(R), \mathbf{Z} / 2 \mathbf{Z}) \geq \frac{1}{2} \operatorname{dim}_{\mathbf{Z} / 2 \mathbf{Z}} H^{1}(X(R), \mathbf{Z} / 2 \mathbf{Z}) .
$$

In particular, if $H^{1}(X(R), \mathbf{Z} / 2 \mathbf{Z}) \neq 0$, then $H_{\text {alg }}^{1}(X(R), \mathbf{Z} / 2 \mathbf{Z}) \neq 0$.

Proof. By Corollary 3.17 (i), we have $H_{\mathrm{alg}}^{1}(X(R), \mathbf{Z} / 2 \mathbf{Z})^{\perp} \subseteq H_{\mathrm{alg}}^{1}(X(R), \mathbf{Z} / 2 \mathbf{Z})$.

Remarks 3.21. (i) This bound is sharp: if $E$ is an elliptic curve over $\mathbf{R}$, if $X \rightarrow E$ is a conic bundle surface with smooth fibres over $E(\mathbf{R})$, and if $X(\mathbf{R})$ is connected and nonempty while $E(\mathbf{R})$ has two connected components, it is an exercise to check that $\operatorname{dim}_{\mathbf{Z} / 2 \mathbf{Z}} H^{1}(X(\mathbf{R}), \mathbf{Z} / 2 \mathbf{Z})=2$ while $\operatorname{dim}_{\mathbf{Z} / 2 \mathbf{Z}} H_{\text {alg }}^{1}(X(\mathbf{R}), \mathbf{Z} / 2 \mathbf{Z})=1$.

(ii) There are surfaces $X$ over $\mathbf{R}$ with $H^{2}\left(X, \mathscr{O}_{X}\right) \neq 0$ (e.g., $K 3$ surfaces) such that $H^{1}(X(\mathbf{R}), \mathbf{Z} / 2 \mathbf{Z}) \neq 0$ but $H_{\text {alg }}^{1}(X(\mathbf{R}), \mathbf{Z} / 2 \mathbf{Z})=0$ (see [Man17, Exemple 4.5.9]).

3.6.3. A criterion for the real integral Hodge conjecture. In the next statements, the real integral Hodge conjecture over an arbitrary real closed field is meant in the sense of Definition 2.3. Similarly, when $R \neq \mathbf{R}$, "the complex integral Hodge conjecture for 1-cycles on $X_{C}$ " when $H^{2}\left(X, \mathscr{O}_{X}\right)=0$ simply refers to the surjectivity of the cycle class map $\mathrm{cl}_{C}: \mathrm{CH}_{1}\left(X_{C}\right) \rightarrow H^{2 d-2}(X(C), \mathbf{Z}(d-1))$. 
Theorem 3.22. Let $X$ be a smooth, proper and geometrically irreducible variety over a real closed field $R$. Assume that $\operatorname{Pic}\left(X_{C}\right)[2]=0$, that $H^{2}\left(X, \mathscr{O}_{X}\right)=0$, and that $X_{C}$ satisfies the complex integral Hodge conjecture for 1-cycles. Then $X$ satisfies the real integral Hodge conjecture for 1-cycles if and only if the following hold:

(i) if $X(R) \neq \varnothing$, then $H_{1}(X(R), \mathbf{Z} / 2 \mathbf{Z})=H_{1}^{\text {alg }}(X(R), \mathbf{Z} / 2 \mathbf{Z})$;

(ii) if $X(R)=\varnothing$, then $X$ contains a geometrically irreducible curve of even geometric genus.

Proof. The exact sequence of Proposition 3.14 fits into a commutative diagram

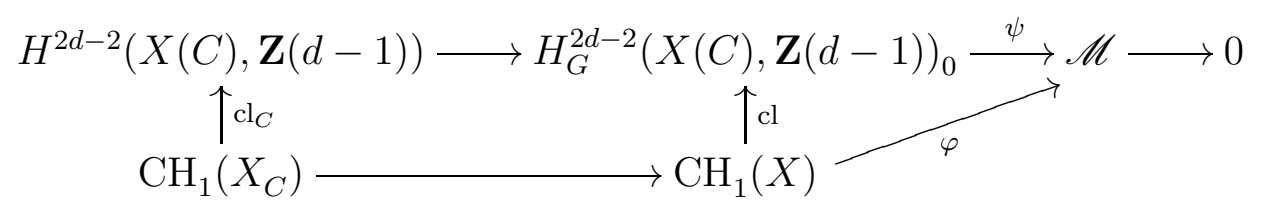

whose bottom horizontal map is the proper push-forward. The map $\mathrm{cl}_{C}$ is surjective by assumption. Hence the surjectivity of $\mathrm{cl}$ is equivalent to that of $\varphi$. The latter is, in turn, equivalent to (i)-(ii), by Theorem 3.6 .

For smooth proper threefolds $X$ that are rationally connected, or Calabi-Yau (in the sense that $K_{X} \simeq \mathscr{O}_{X}$ and $\left.H^{1}\left(X, \mathscr{O}_{X}\right)=H^{2}\left(X, \mathscr{O}_{X}\right)=0\right)$, the complex integral Hodge conjecture for 1-cycles was proved by Voisin [Voi06, Theorem 2] when $R=\mathbf{R}$. Using comparison with étale cohomology and the Lefschetz principle, the same holds for an arbitrary real closed field $R$. One deduces:

Corollary 3.23. Let $X$ be a smooth and proper threefold over a real closed field $R$. Assume that $X$ is rationally connected or is simply connected Calabi-Yau. Then the real integral Hodge conjecture for $X$ is equivalent to the equality $H_{1}^{\text {alg }}(X(R), \mathbf{Z} / 2 \mathbf{Z})=$ $H_{1}(X(R), \mathbf{Z} / 2 \mathbf{Z})$, if $X(R) \neq \varnothing$, or to the existence of a geometrically irreducible curve of even geometric genus, if $X(R)=\varnothing$.

\section{EXAmples}

We now provide various examples of smooth, proper and geometrically irreducible varieties $X$ over $\mathbf{R}$ such that one of the two equalities $\operatorname{ind}_{1}(X)=1($ when $X(\mathbf{R})=\varnothing)$ or $H_{1}(X(\mathbf{R}), \mathbf{Z} / 2 \mathbf{Z})=H_{1}^{\text {alg }}(X(\mathbf{R}), \mathbf{Z} / 2 \mathbf{Z})$ (when $\left.X(\mathbf{R}) \neq \varnothing\right)$ fails. We recall that when $X(\mathbf{R})=\varnothing$, the condition $\operatorname{ind}_{1}(X)=1$ is equivalent to the existence of a curve of even genus in $X$ (see Corollary 3.11). All of these examples illustrate the obstructions to the surjectivity of $\varphi: \mathrm{CH}_{1}(X) \rightarrow \mathscr{M}$ described in $\$ 3.6 .1$.

4.1. Topological obstructions. The easiest examples are curves of positive genus. For a curve, only a topological obstruction can prevent $\varphi$ from being surjective, since the map cl : $\mathrm{CH}_{1}(X) \rightarrow H_{G}^{2 d-2}(X(C), \mathbf{Z}(d-1))_{0}$ is an isomorphism when $d=1$. 
Example 4.1 (with real points). If $X$ is a curve and $X(\mathbf{R})$ has at least two connected components, then $H_{1}(X(\mathbf{R}), \mathbf{Z} / 2 \mathbf{Z}) \neq H_{1}^{\text {alg }}(X(\mathbf{R}), \mathbf{Z} / 2 \mathbf{Z})$.

Example 4.2 (with no real point). If $X$ is a curve of odd genus and $X(\mathbf{R})=\varnothing$, then $\operatorname{ind}_{1}(X)=2$ (see Corollary 3.11).

By Theorem 3.3, topological obstructions can only occur if $\operatorname{Pic}\left(X_{\mathbf{C}}\right)[2] \neq 0$. Curves of positive genus satisfy the stronger property that the abelian variety $\operatorname{Pic}^{0}\left(X_{\mathbf{C}}\right)$ is non-zero. Let us now show that surfaces with $\operatorname{Pic}^{0}\left(X_{\mathbf{C}}\right)=0$ can also carry topological obstructions. The surfaces used in the next two examples even satisfy $H^{i}\left(X, \mathscr{O}_{X}\right)=0$ for all $i>0$ : they illustrate the sharpness of the last statement of Corollary 3.17

Example 4.3 (with real points). Let $X$ be a real Enriques surface such that $X(\mathbf{R})$ is non-orientable (such surfaces exist; see, e.g., [DK96, Theorem 2.2]). By the theorem of Mangolte and van Hamel [MvH98, Theorem 1.1] recalled in Remark 3.18 (ii), we then have $H_{1}(X(\mathbf{R}), \mathbf{Z} / 2 \mathbf{Z}) \neq H_{1}^{\text {alg }}(X(\mathbf{R}), \mathbf{Z} / 2 \mathbf{Z})$. This is explained by a topological obstruction (see Remark 3.18 (ii) and the discussion in \$3.6.2).

As we have seen in Example 3.19, the map $\varphi$ is always surjective for an Enriques surface with no real point. To provide an analogue of Example 4.3 in the case with no real point, we resort to Campedelli surfaces instead. These are minimal surfaces of general type with $H^{i}\left(X, \mathscr{O}_{X}\right)=0$ for $i>0$ and $K_{X}^{2}=2$. The precise surfaces we use below were constructed, over C, by Godeaux [God49, §6], and are also described in [Rei79, §2.1]. We endow them with an appropriate real structure.

Example 4.4 (with no real point). Let $\zeta$ be a primitive eighth root of unity. Let $f: \mathbf{P}^{6}(\mathbf{C}) \rightarrow \mathbf{P}^{6}(\mathbf{C})$ be the map defined by

$$
f\left(\left[x_{0}: \cdots: x_{6}\right]\right)=\left[\zeta \overline{x_{1}}: \overline{x_{0}}: \zeta^{2} \overline{x_{3}}: \overline{x_{2}}: \zeta^{3} \overline{x_{5}}: \overline{x_{4}}: \overline{x_{6}}\right] .
$$

Let $H$ denote the group of diffeomorphisms of $\mathbf{P}^{6}(\mathbf{C})$ generated by $f$. One checks that $H=\mathbf{Z} / 16 \mathbf{Z}$ and that the index 2 subgroup $K=\mathbf{Z} / 8 \mathbf{Z} \subset H$ acts holomorphically on $\mathbf{P}^{6}(\mathbf{C})$ while the other elements of $H$ act antiholomorphically. Let us identify $\left(f^{2}\right)^{*} \mathscr{O}(1)$ with $\mathscr{O}(1)$ by mapping $x_{0}$ to $\zeta x_{0}$ and let us consider the endomorphism of the complex vector space $H^{0}\left(\mathbf{P}^{6}(\mathbf{C}), \mathscr{O}(2)\right)$ induced by $f^{2}$ and by this identification. The family $\left(x_{i} x_{j}\right)_{0<i<j<6}$ forms an eigenbasis. Let $\Lambda_{i}$ denote the eigenspace associated with $\zeta^{2 i}$. For $\left(Q_{0}, \ldots, Q_{3}\right) \in \prod_{i=0}^{3} \Lambda_{i}$, let $Y \subset \mathbf{P}^{6}(\mathbf{C})$ denote the subvariety defined by $Q_{0}=\cdots=Q_{3}=0$. By an explicit computation based on the Bertini theorem for linear systems, one checks that if $Q_{0}, \ldots, Q_{3}$ are general, then $Y$ is a smooth surface that does not meet the fixed locus of $f^{8}$, hence does not meet the fixed locus of any nontrivial element of $H$. Moreover, one checks that there is a Zariski dense set of quadruples $\left(Q_{0}, \ldots, Q_{3}\right)$ for which $Y$ is stable under $f$. As a consequence, we can choose $\left(Q_{0}, \ldots, Q_{3}\right)$ such that $Y$ is a smooth surface on which $H$ acts freely. The quotient $S=Y / K$ is then a smooth projective complex surface on which $f$ induces a 
fixed-point free antiholomorphic involution $\sigma$. There exist a real projective surface $X$ and an isomorphism $X(\mathbf{C}) \simeq S$ through which complex conjugation corresponds to $\sigma$ (see [Sil89, Proposition I.1.4]). We then have $X(\mathbf{R})=\varnothing$. As $Y$ is a simply connected étale cover of both $X_{\mathbf{C}}$ and $X$, we have $\pi_{1}^{\text {ét }}\left(X_{\mathbf{C}}\right)=K$ and $\pi_{1}^{\text {ét }}(X)=H$, hence $H_{\text {ét }}^{1}\left(X_{\mathbf{C}}, \mathbf{Z} / 2 \mathbf{Z}\right)=\mathbf{Z} / 2 \mathbf{Z}$ and $H_{\text {ét }}^{1}(X, \mathbf{Z} / 2 \mathbf{Z})=\mathbf{Z} / 2 \mathbf{Z}$. By the Kummer exact sequence, we deduce that $\operatorname{Pic}\left(X_{\mathbf{C}}\right)[2]=\mathbf{Z} / 2 \mathbf{Z}$ and $\operatorname{Pic}(X)[2]=0$. Corollary [3.17 (ii) now implies that $\operatorname{ind}_{1}(X)=2$.

4.2. Hodge-theoretic obstructions. The next two examples are $K 3$ surfaces. As such surfaces are simply connected, they cannot carry a topological obstruction; however, they may carry a Hodge-theoretic obstruction. These examples illustrate the importance of the hypothesis $H^{2}\left(X, \mathscr{O}_{X}\right)=0$ in Corollary 3.17 .

Example 4.5 (with real points). Examples of quartic surfaces $X \subset \mathbf{P}_{\mathbf{R}}^{3}$ such that $H^{1}(X(\mathbf{R}), \mathbf{Z} / 2 \mathbf{Z}) \neq H_{\text {alg }}^{1}(X(\mathbf{R}), \mathbf{Z} / 2 \mathbf{Z})$ are given in $[\mathrm{BK} 89$, Example 3.4 (c) and (d)] and [Man17, Exemple 4.5.9]. As a further example, take $X$ to be a very general small real deformation of a smooth quartic surface containing a real line. The cohomology class of the line deforms to a class $\alpha \in H^{1}(X(\mathbf{R}), \mathbf{Z} / 2 \mathbf{Z})$ such that $\operatorname{deg}\left(\alpha \smile \operatorname{cl}_{\mathbf{R}}\left(\mathscr{O}_{X}(1)\right)\right) \neq 0 \in \mathbf{Z} / 2 \mathbf{Z}$ by Ehresmann's theorem, and $H_{\text {alg }}^{1}(X(\mathbf{R}), \mathbf{Z} / 2 \mathbf{Z})$ is generated by $\mathrm{cl}_{\mathbf{R}}\left(\mathscr{O}_{X}(1)\right)$ by the Noether-Lefschetz theorem. Since $\operatorname{deg}\left(\operatorname{cl}_{\mathbf{R}}\left(\mathscr{O}_{X}(1)\right) \smile \operatorname{cl}_{\mathbf{R}}\left(\mathscr{O}_{X}(1)\right)\right)=0 \in \mathbf{Z} / 2 \mathbf{Z}$, one has $\alpha \notin H_{\text {alg }}^{1}(X(\mathbf{R}), \mathbf{Z} / 2 \mathbf{Z})$.

Example 4.6 (with no real point). Let $X \subset \mathbf{P}_{\mathbf{R}}^{3}$ be a very general quartic surface such that $X(\mathbf{R})=\varnothing$. As $\mathrm{CH}_{1}(X)$ is generated by the class of a hyperplane section, which has genus 3, Theorem 3.6 immediately implies that $X$ does not contain any geometrically irreducible curve of even geometric genus. This example was first noted by Kollár, see [CTM04, p. 15, Remarque (3)].

4.3. Cycle-theoretic obstructions (failures of the real integral Hodge conjecture). We now give examples, with or without real points, of cycle-theoretic obstructions to the surjectivity of $\varphi$. In view of Proposition 2.8, such obstructions cannot occur on curves or surfaces. The examples we give are simply connected threefolds which satisfy $H^{2}\left(X, \mathscr{O}_{X}\right)=0$. As such, they cannot carry a topological or Hodge-theoretic obstruction. Their construction relies on Kollár's specialisation method to provide counterexamples to the integral Hodge conjecture (see [BCC92]), as implemented by Totaro [Tot13. To the best of our knowledge, Example 4.7 constitutes a new kind of example of a real variety $X$ such that $H_{1}(X(\mathbf{R}), \mathbf{Z} / 2 \mathbf{Z}) \neq$ $H_{1}^{\text {alg }}(X(\mathbf{R}), \mathbf{Z} / 2 \mathbf{Z})$ (see Remark 2.7 (i)). Here, the underlying phenomenon is a defect of the complex integral Hodge conjecture.

Example 4.7 (with real points). The example will be a hypersurface $X \subset \mathbf{P}_{\mathbf{Q}}^{1} \times \mathbf{P}_{\mathbf{Q}}^{3}$ of bidegree $(4,4)$. Let us first fix a prime number $p$ (for instance $p=2$ ) and choose $f_{p} \in \mathbf{Z}\left[u, v, x_{0}, \ldots, x_{3}\right]$, bihomogeneous of bidegree $(4,4)$ in $(u, v),\left(x_{0}, \ldots, x_{3}\right)$, whose 
zero locus $X_{f_{p}} \subset \mathbf{P}_{\mathbf{Q}}^{1} \times \mathbf{P}_{\mathbf{Q}}^{3}$ is smooth and whose reduction modulo $p$ is equal to $v\left(u^{3} x_{0}^{4}+u^{2} v x_{1}^{4}+u v^{2} x_{2}^{4}+v^{3} x_{3}^{4}\right)$. Let us choose $f_{\mathbf{R}} \in \mathbf{Q}\left[u, v, x_{0}, \ldots, x_{3}\right]$, bihomogeneous of bidegree $(4,4)$, such that $X_{f_{\mathbf{R}}} \subset \mathbf{P}_{\mathbf{Q}}^{1} \times \mathbf{P}_{\mathbf{Q}}^{3}$ is a smooth hypersurface containing $\mathbf{P}_{\mathbf{Q}}^{1} \times\{[1: 0: 0: 0]\}$. Such $f_{\mathbf{R}}$ exist as a consequence of the refined Bertini theorem of [KA79, Theorem 7]. Finally, let $f \in \mathbf{Q}\left[u, v, x_{0}, \ldots, x_{3}\right]$ be arbitrarily close to $f_{\mathbf{R}}$ in the real topology and to $f_{p}$ in the $p$-adic topology, and let $X=X_{f}$. As $f$ is $p$-adically close to $f_{p}$, the argument used in the proof of [Tot13, Theorem 3.1] shows that every curve in $X_{\mathbf{C}}$ has even degree over $\mathbf{P}_{\mathbf{C}}^{1}$. A fortiori, every curve in $X$ has even degree over $\mathbf{P}_{\mathbf{R}}^{1}$ : the push-forward map $H_{1}(X(\mathbf{R}), \mathbf{Z} / 2 \mathbf{Z}) \rightarrow H_{1}\left(\mathbf{P}^{1}(\mathbf{R}), \mathbf{Z} / 2 \mathbf{Z}\right)$ must vanish on $H_{1}^{\text {alg }}(X(\mathbf{R}), \mathbf{Z} / 2 \mathbf{Z})$. On the other hand, as $f$ is close to $f_{\mathbf{R}}$, this push-forward map is surjective. (Its surjectivity is indeed invariant under small real deformations, by Ehresmann's theorem.) Hence $H_{1}(X(\mathbf{R}), \mathbf{Z} / 2 \mathbf{Z}) \neq H_{1}^{\text {alg }}(X(\mathbf{R}), \mathbf{Z} / 2 \mathbf{Z})$.

Example 4.8 (with no real point). Let $B$ be a smooth, projective conic over $\mathbf{Q}$ such that $B(\mathbf{R})=\varnothing$. Let $p$ be a prime of good reduction for $B$, i.e., such that there exists a smooth $\mathbf{Z}_{(p)}$-scheme $\mathscr{B}$ with generic fiber $B$ and special fiber $\mathbf{P}_{\mathbf{F}_{p}}^{1}$. Let $\mathscr{Y}=\mathscr{B} \times \mathbf{P}^{3}$, $Y=B \times \mathbf{P}^{3}$, and $\mathscr{Y}_{0}=\mathscr{Y} \otimes \mathbf{F}_{p}=\mathbf{P}_{\mathbf{F}_{p}}^{1} \times \mathbf{P}_{\mathbf{F}_{p}}^{3}$. Let $\mathscr{O}_{\mathscr{Y}}(4,4)$ be the dual of $\omega_{\mathscr{B}}^{\otimes 2} \otimes \omega_{\mathbf{P}^{3}}$. As $H^{1}\left(\mathscr{Y}, \mathscr{O}_{\mathscr{Y}}(4,4)\right)=0$, the restriction map $H^{0}\left(\mathscr{Y}, \mathscr{O}_{\mathscr{Y}}(4,4)\right) \rightarrow H^{0}\left(\mathscr{Y}_{0}, \mathscr{O}_{\mathscr{Y}}(4,4)\right)$ is onto. In addition, its kernel is Zariski dense in $H^{0}\left(Y, \mathscr{O}_{Y}(4,4)\right)$ (viewed as an affine space over $\mathbf{Q})$. We can therefore choose a section $f \in H^{0}\left(\mathscr{Y}, \mathscr{O}_{\mathscr{Y}}(4,4)\right)$ which reduces to $v\left(u^{3} x_{0}^{4}+u^{2} v x_{1}^{4}+u v^{2} x_{2}^{4}+v^{3} x_{3}^{4}\right) \in H^{0}\left(\mathscr{Y}_{0}, \mathscr{O}_{\mathscr{Y}_{0}}(4,4)\right)$ modulo $p$, where $[u: v]$ and $\left[x_{0}: x_{1}: x_{2}: x_{3}\right]$ respectively denote the homogeneous coordinates of $\mathbf{P}_{\mathbf{F}_{p}}^{1}$ and of $\mathbf{P}_{\mathbf{F}_{p}}^{3}$, and such that the zero locus $X_{f} \subset B \times \mathbf{P}^{3}$ is smooth over $\mathbf{Q}$. Let $X=X_{f} \otimes_{\mathbf{Q}} \mathbf{R}$. The argument used in the proof of [Tot13, Theorem 3.1] shows that every curve in $X_{\mathbf{C}}$ has even degree over $B_{\mathbf{C}}$. A fortiori, every curve in $X$ has even degree over $B_{\mathbf{R}}$. By Proposition 3.12 , it follows that every curve in $X$ has odd genus, so that $\operatorname{ind}_{1}(X)=2$.

In view of Examples 4.7 and 4.8, it is natural to ask:

Question 4.9. Does there exist a smooth, proper and geometrically irreducible variety $X$ over $\mathbf{R}$ such that $X_{\mathbf{C}}$ satisfies the complex integral Hodge conjecture for 1 -cycles, but such that $X$ has a cycle-theoretic obstruction to the surjectivity of $\varphi$ ?

Although we do not know any variety over $\mathbf{R}$ with these properties, we will exhibit such examples over non-archimedean real closed fields in [BW18, §9.2].

\section{Bloch-Ogus theORY AND TORSION 1-CYCLES}

In this section, we apply Bloch-Ogus theory to investigate the consequences of the real integral Hodge conjecture for the study of the group $\mathrm{CH}_{1}(X)_{\text {tors }}$ and of its image in $H_{1}(X(R), \mathbf{Z} / 2 \mathbf{Z})$ by the Borel-Haefliger cycle class map. The main result is Theorem 5.4. We illustrate its applicability in \$5.4 by computing $\mathrm{CH}_{1}(X)_{\text {tors }}$ for a 
smooth quartic threefold $X$ over a real closed field $R$, under the assumption that $X$ satisfies the real integral Hodge conjecture and that $X(R)=\varnothing$. As a preliminary step, we prove, in $\$ 5.2$, that the defect of the real integral Hodge conjecture, for a threefold, can be interpreted in terms of unramified cohomology; this is a real analogue of a theorem of Colliot-Thélène and Voisin [CTV12. Along the way, we also obtain some new technical results of independent interest in $\$ 5.1$, such as the vanishing of the upper differentials in the coniveau spectral sequence for the equivariant cohomology of $X(C)$ with coefficients in an arbitrary $G$-module $M$ (see Proposition 5.1 (vi)), an assertion which was previously known only when $M$ is one of $\mathbf{Z} / 2 \mathbf{Z}, \mathbf{Q} / \mathbf{Z}, \mathbf{Q} / \mathbf{Z}(1)$ (due to the work of Colliot-Thélène, Parimala, Scheiderer, van Hamel; see [CTP90, §3.1], [Sch94, Proposition 19.8], [CTS96, §3], vH00b, §2]).

5.1. Complements on Bloch-Ogus theory. We collect, in $\$ 5.1$, some results on Bloch-Ogus theory in the context of equivariant semi-algebraic cohomology. General references for this theory are [BO74] and [CTHK97]. It was applied in real algebraic geometry in [CTP90], Sch94, Chapter 19], CTS96], vH00b], HV12] with Z/2Z coefficients and in [Ben16, §2] with $\mathbf{Z}_{2}$ coefficients.

We fix a $G$-module $M$. Applying [CTHK97, Remark 5.1.3 (3)] to the cohomology theory with supports $(X, Z) \mapsto H_{G, Z(C)}^{*}(X(C), M)$, we obtain the coniveau spectral sequence, which, in view of (1.21), takes the form

$$
E_{1}^{p, q}=\bigoplus_{Z \subseteq X} \underset{\lim _{U \subseteq Z}}{\rightarrow} H_{G}^{q-p}(U(C), M(-p)) \Rightarrow H_{G}^{p+q}(X(C), M)
$$

for any smooth variety $X$ over $R$; here, the direct sum ranges over the irreducible Zariski closed subsets $Z \subseteq X$ of codimension $p$ and the direct limit ranges over the dense Zariski open subsets $U \subseteq Z$. Clearly $E_{r}^{p, q}=0$ for all $r \geq 1$ whenever $p>q$.

This cohomology theory with supports satisfies the étale excision and homotopy invariance axioms of [CTHK97, §5.1, §5.3], as a consequence of the semi-algebraic implicit function theorem (see [DK84, Example 5.1]), for étale excision, and of (1.17) and [Del85, Corollary 4.5], for homotopy invariance. By CTHK97, Corollary 5.1.11 and Proposition 5.3.2], we deduce, for any $p, q$, a canonical isomorphism

$$
E_{2}^{p, q}=H^{p}\left(X, \mathscr{H}_{X}^{q}(M)\right)
$$

if $\mathscr{H}_{X}^{q}(M)$ denotes the Zariski sheaf associated with the presheaf $U \mapsto H_{G}^{q}(U(C), M)$.

We denote by $N^{p} H_{G}^{i}(X(C), M) \subseteq H_{G}^{i}(X(C), M)$ the subgroup of those classes $\alpha$ for which there exists a Zariski closed subset $Z \subseteq X$ of codimension $\geq p$ such that $\alpha$ is supported on $Z(C)$. The filtration thus defined, called the coniveau filtration, is the one determined by (5.1).

The preceding discussion also applies with equivariant cohomology replaced by cohomology (or we may simply apply it to $X_{C}$ viewed as a geometrically reducible variety over $R$ in the naive way). We let $\pi: X_{C} \rightarrow X$ denote the projection map and 
$\mathscr{H}_{X_{C}}^{q}(M)$ the Zariski sheaf, on $X_{C}$, associated with the presheaf $U \mapsto H^{q}(U(C), M)$. The next proposition summarises the analogues, for semi-algebraic cohomology with coefficients in $\mathbf{Z}$, of some of the statements of [Ben16, §2] (assertions (i), (ii), (iii)) and of [Sch94, Chapter 19] and [vH00b, §1.7-§2] (assertions (iv), (v), (vi)).

Proposition 5.1. Let $X$ be a smooth variety over $R$, of dimension d.

(i) Let $M$ be a $G$-module. For every $p, q \geq 0$, there are canonical isomorphisms $\mathscr{H}_{X}^{q}(M[G])=\pi_{*} \mathscr{H}_{X_{C}}^{q}(M)$ and $H^{p}\left(X, \pi_{*} \mathscr{H}_{X_{C}}^{q}(M)\right)=H^{p}\left(X_{C}, \mathscr{H}_{X_{C}}^{q}(M)\right)$.

(ii) For every $q \geq 0$, the sheaves $\mathscr{H}_{X}^{q}(\mathbf{Z}(q-1))$ and $\mathscr{H}_{X}^{q}(\mathbf{Z}[G])$ are torsion-free.

(iii) The real-complex exact sequence (1.1) induces an exact sequence

$$
\frac{0 \rightarrow \mathscr{H}_{X}^{q}(\mathbf{Z}(q-1)) \rightarrow \pi_{*} \mathscr{H}_{X_{C}}^{q}(\mathbf{Z}) \rightarrow \mathscr{H}_{X}^{q}(\mathbf{Z}(q))}{\rightarrow \mathscr{H}_{X}^{q+1}(\mathbf{Z}(q-1)) \rightarrow \pi_{*} \mathscr{H}_{X_{C}}^{q+1}(\mathbf{Z}) \rightarrow \mathscr{H}_{X}^{q+1}(\mathbf{Z}(q)) \rightarrow 0}
$$

for every $q \geq 0$.

(iv) Let $q>d$. Let $M$ be a $G$-module. Let us denote by $\iota: X(R) \rightarrow X$ the natural morphism of sites from the semi-algebraic site of $X(R)$ to the small Zariski site of $X$. The natural map $\mathscr{H}_{X}^{q}(M) \rightarrow \iota_{*} H^{q}(G, M)$ is an isomorphism. In addition, one has $H^{p}\left(X, \mathscr{H}_{X}^{q}(M)\right)=H^{p}\left(X(R), H^{q}(G, M)\right)$ for all $p \geq 0$.

(v) For $j \in \mathbf{Z}$ and $i \geq 0$, the restriction map $H_{G}^{i}(X(C), \mathbf{Z}(j)) \rightarrow H_{G}^{i}(X(R), \mathbf{Z}(j))$ and the decomposition (1.30) induce an isomorphism

$$
H_{G}^{i}(X(C), \mathbf{Z}(j)) / N^{i-d} H_{G}^{i}(X(C), \mathbf{Z}(j)) \stackrel{\sim}{\longrightarrow} \bigoplus_{\substack{0 \leq p<i-d \\ p \equiv i-j \bmod 2}} H^{p}(X(R), \mathbf{Z} / 2 \mathbf{Z}) .
$$

(vi) The differential $E_{r}^{p, q} \rightarrow E_{r}^{p+r, q-r+1}$ of the coniveau spectral sequence (5.1) vanishes for all $p, q, r$ such that $r \geq 2$ and $q>d$ and for any $G$-module $M$.

Proof. The first isomorphism of (i) follows from (1.5). The second one is obtained in [CTS96, Lemma 2.2.1 (a)] with $\mathbf{Z} / 2 \mathbf{Z}$ coefficients and in [Ben16, Proposition 2.1] in the setting of 2-adic cohomology. The arguments given there apply verbatim with equivariant semi-algebraic cohomology with coefficients in $M$.

For any prime number $\ell$, the sheaf associated with $U \mapsto H_{\text {ét }}^{q}\left(U, \mathbf{Z}_{\ell}(q-1)\right)$ is torsion-free (see [Ben16, Proposition 2.2], where the assumption that $\ell=2$ is not used; the underlying argument, which rests on the Bloch-Kato conjecture, goes back to [BS83, Proof of Theorem 1] and to [CTV12, Théorème 3.1] and does not depend on the nature of the ground field $R$ ). By the comparison between equivariant semialgebraic cohomology and $\ell$-adic cohomology, it follows that the sheaf $\mathscr{H}_{X}^{q}(\mathbf{Z}(q-1))$ is torsion-free. Applying this to $X_{C}$ and noting that $\mathscr{H}_{X}^{q}(\mathbf{Z}[G])=\pi_{*} \mathscr{H}_{X_{C}}^{q}(\mathbf{Z}(q-1))$, we see that $\mathscr{H}_{X}^{q}(\mathbf{Z}[G])$ is torsion-free as well. The proof of (ii) is complete.

Assertion (iii) follows from (i) and (ii). This is observed in [Ben16, Proposition 2.5] for 2-adic cohomology and the same proof applies here. 
Assertion (iv) for torsion $G$-modules $M$ is due to Scheiderer Sch94, Corollary 6.9.1 and Corollary 19.5]. We adapt his arguments to an arbitrary $G$-module $M$ as follows. The restriction map $H_{G}^{q}(U(C), M) \rightarrow H_{G}^{q}(U(R), M)$ is an isomorphism for any $q>d$ and any affine open subset $U \subseteq X$, by Lemma 1.16. In addition, the map $H_{G}^{q}(U(R), M) \rightarrow H^{0}\left(U(R), H^{q}(G, M)\right)$ induced by the spectral sequence

$$
E_{2}^{a, b}(U)=H^{a}\left(U(R), H^{b}(G, M)\right) \Rightarrow H_{G}^{a+b}(U(R), M)
$$

(see (1.3) ) becomes an isomorphism after sheafification with respect to $U$, since the sheaf associated with the presheaf $U \mapsto E_{2}^{a, b}(U)$ vanishes when $a>0$ according to [Sch94, Proposition 19.2.1] (see also the proof of [vH00b, Lemma 1.2]). All in all, we obtain the desired isomorphism $\mathscr{H}_{X}^{q}(M) \stackrel{\sim}{\longrightarrow} \iota_{*} H^{q}(G, M)$. On the other hand, the functor $\iota_{*}$ is exact, by [Sch94, Theorem 19.2]. By the Leray spectral sequence for $\iota$, assertion (iv) follows.

Assertion (vi) for $M=\mathbf{Z} / 2 \mathbf{Z}$ is due to van Hamel [vH00b, Theorem 2.1]. Based on Proposition 1.8, we extend his arguments to integral coefficients. In fact, we shall prove (v) and (vi) for $M=\mathbf{Z}(j)$ simultaneously. To this end, we first note that by purity for semi-algebraic cohomology, the group $H_{Z(R)}^{p}(X(R), \mathbf{Z} / 2 \mathbf{Z})$ vanishes for any closed subset $Z \subseteq X$ of codimension $>p$. (See (1.13) and the proof of [Mil80, Chapter VI, Lemma 9.1].) Hence the restriction map induces a map

$$
H_{G}^{i}(X(C), \mathbf{Z}(j)) / N^{i-d} H_{G}^{i}(X(C), \mathbf{Z}(j)) \rightarrow \underset{\substack{0 \leq p<i-d \\ p \equiv i-j \bmod 2}}{\rightarrow} H^{p}(X(R), \mathbf{Z} / 2 \mathbf{Z}),
$$

which is surjective by Proposition 1.8. Let us now consider the coniveau spectral sequence (5.1) associated with $M=\mathbf{Z}(j)$. We have $E_{2}^{p, q}=H^{p}\left(X(R), H^{q}(G, \mathbf{Z}(j))\right)$ whenever $q>d$, according to (iv) and to (5.2). The target of (5.3) can therefore be rewritten as $\bigoplus_{0 \leq p<i-d} E_{2}^{p, i-p}$. On the other hand, the domain of (5.3) has the same cardinality as $\bigoplus_{0 \leq p<i-d} E_{\infty}^{p, i-p}$, which is finite. As $E_{\infty}^{p, i-p}$ is a subquotient of $E_{2}^{p, i-p}$ for all $p$ and as (15.3) is surjective, it follows that (15.3) is an isomorphism and that $E_{\infty}^{p, i-p}=E_{2}^{p, i-p}$ for $p<i-d$. We have thus established (v), as well as (vi) for $M=\mathbf{Z}(j)$ (with $q=i-p)$.

It remains to check (vi) for an arbitrary $G$-module $M$, which we now fix. The map $H^{q}(G, N(j)) \rightarrow H^{q}(G, M(j))$ induced by the natural morphism of $G$-modules $N=$ $\mathbf{Z}^{\left(H^{0}(G, M)\right)} \oplus \mathbf{Z}(1)^{\left(H^{0}(G, M(1))\right)} \rightarrow M$ is surjective for $q=0$ and any $j$, therefore also for any $q$ and any $j$ since the boundary map $H^{q-1}(G, M(j+1)) \rightarrow H^{q}(G, M(j))$ of (1.1) is surjective when $q>0$ (see Bro94, Chapter III, Corollary 5.7, Proposition 5.9, Corollary 6.6]). For $q>0$, this is a surjection between $\mathbf{Z} / 2 \mathbf{Z}$-vector spaces, hence it is a split surjection. We deduce that the group $H^{p}\left(X(R), H^{q}(G, N)\right)$ surjects onto $H^{p}\left(X(R), H^{q}(G, M)\right)$ for all $p \geq 0$ and all $q>0$. This remark, together with 
assertion (iv) for the $G$-modules $M$ and $N$ and with assertion (vi) for the $G$-module $N$ (which we have already shown to hold) implies assertion (vi) for $M$.

5.2. Coniveau and the real integral Hodge conjecture for 1-cycles. If $X$ is a smooth, proper and irreducible complex variety of dimension $d$ such that $\mathrm{CH}_{0}(X)$ is supported on a surface (i.e., such that there exists a closed subvariety $Y \subseteq X$ of dimension $\leq 2$ such that $\mathrm{CH}_{0}(Y) \rightarrow \mathrm{CH}_{0}(X)$ ), Colliot-Thélène and Voisin CTV12, Corollaire 3.12] have shown that the defect of the integral Hodge conjecture for 1-cycles on $X$ is measured by the group $H^{d-3}\left(X, \mathscr{H}_{X}^{d}(\mathbf{Q} / \mathbf{Z}(d-1))\right)$. We establish, in Proposition 5.2 below, an analogue of this statement for real varieties.

For a variety $X$ defined over a real closed field $R$, a $G$-module $M$ and any $q \geq 0$, we set $\mathscr{H}_{X}^{q}(M)_{0}=\operatorname{Ker}\left(\mathscr{H}_{X}^{q}(M) \rightarrow \iota_{*} H^{q}(G, M)\right.$ ) (notation as in Proposition 5.1 (iv)).

Proposition 5.2. Let $X$ be a smooth, proper and geometrically irreducible variety, of dimension d, over a real closed field $R$. If $\mathrm{CH}_{0}\left(X_{C^{\prime}}\right)$ is supported on a surface for every algebraically closed field $C^{\prime}$ containing $R$, there is a canonical isomorphism

$$
H^{d-3}\left(X, \mathscr{H}_{X}^{d}(\mathbf{Q} / \mathbf{Z}(d-1))_{0}\right)=\operatorname{Coker}\left(\mathrm{CH}_{1}(X) \rightarrow H_{G}^{2 d-2}(X(C), \mathbf{Z}(d-1))_{0}\right)_{\text {tors }} \text {. }
$$

If moreover $R=\mathbf{R}$ or $H^{2}\left(X, \mathscr{O}_{X}\right)=0$, the real integral Hodge conjecture (see \$2.2) holds for 1 -cycles on $X$ if and only if $H^{d-3}\left(X, \mathscr{H}_{X}^{d}(\mathbf{Q} / \mathbf{Z}(d-1))_{0}\right)=0$.

Proof. Let us consider the commutative square

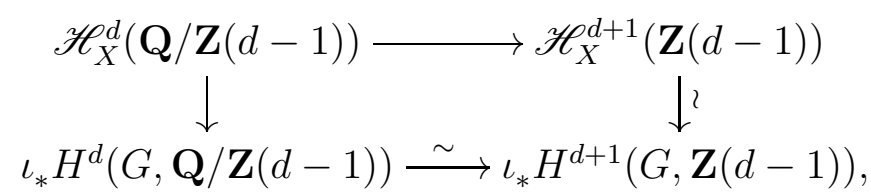

whose horizontal maps come from the short exact sequence $0 \rightarrow \mathbf{Z} \rightarrow \mathbf{Q} \rightarrow \mathbf{Q} / \mathbf{Z} \rightarrow 0$. The vertical map on the right is an isomorphism, by Proposition 5.1 (iv). Hence the kernel of the top horizontal map is $\mathscr{H}_{X}^{d}(\mathbf{Q} / \mathbf{Z}(d-1))_{0}$ and we therefore obtain, in view of Proposition 5.1 (ii), a short exact sequence

$$
0 \longrightarrow \mathscr{H}_{X}^{d}(\mathbf{Z}(d-1)) \longrightarrow \mathscr{H}_{X}^{d}(\mathbf{Q}(d-1)) \longrightarrow \mathscr{H}_{X}^{d}(\mathbf{Q} / \mathbf{Z}(d-1))_{0} \longrightarrow 0
$$

The sheaf $\mathscr{H}_{X}^{d}(\mathbf{Q}(d-1))$ is a direct summand of $\pi_{*} \mathscr{H}_{X_{C}}^{d}(\mathbf{Q})$. By Proposition 5.1 (i), it follows that $H^{d-3}\left(X, \mathscr{H}_{X}^{d}(\mathbf{Q}(d-1))\right)$ injects into $H^{d-3}\left(X_{C}, \mathscr{H}_{X_{C}}^{d}(\mathbf{Q})\right)$. The latter group vanishes since $\mathrm{CH}_{0}\left(X_{C^{\prime}}\right)$ is supported on a surface for every algebraically closed fields $C^{\prime}$ containing $C$ (see [CTV12, Proposition 3.3 (ii)], whose proof goes through over an arbitrary algebraically closed field of characteristic 0 provided one replaces the hypothesis on $\mathrm{CH}_{0}\left(X_{C}\right)$ with the same hypothesis on $\mathrm{CH}_{0}\left(X_{C^{\prime}}\right)$ for all $\left.C^{\prime}\right)$. In view of (5.5), we deduce that

$$
H^{d-3}\left(X, \mathscr{H}_{X}^{d}(\mathbf{Q} / \mathbf{Z}(d-1))_{0}\right)=H^{d-2}\left(X, \mathscr{H}_{X}^{d}(\mathbf{Z}(d-1))\right)_{\text {tors }} .
$$


Let us now consider the coniveau spectral sequence (5.1) for $M=\mathbf{Z}(d-1)$. We have $H^{d-2}\left(X, \mathscr{H}_{X}^{d}(\mathbf{Z}(d-1))\right)=E_{2}^{d-2, d}($ see $(5.2))$. By Proposition 5.1 (vi) and in view of the fact that $E_{1}^{p, q}=0$ for $p>q$, we also have $E_{\infty}^{d-2, d}=E_{2}^{d-2, d}$. On the other hand, we have

$$
N^{d-1} H_{G}^{2 d-2}(X(C), \mathbf{Z}(d-1))=\operatorname{Im}\left(\mathrm{CH}_{1}(X) \rightarrow H_{G}^{2 d-2}(X(C), \mathbf{Z}(d-1))\right)
$$

as a consequence of equivariant purity (see (1.21)) and

$$
N^{d-2} H_{G}^{2 d-2}(X(C), \mathbf{Z}(d-1))=H_{G}^{2 d-2}(X(C), \mathbf{Z}(d-1))_{0}
$$

according to Proposition 5.1 (v) applied with $i=2 d-2, j=d-1$. As the quotient of (5.8) by (5.7) is $E_{\infty}^{d-2, d}$, we conclude that

$$
H^{d-2}\left(X, \mathscr{H}_{X}^{d}(\mathbf{Z}(d-1))\right)=\operatorname{Coker}\left(\mathrm{CH}_{1}(X) \rightarrow H_{G}^{2 d-2}(X(C), \mathbf{Z}(d-1))_{0}\right) .
$$

Together with (5.6) , this proves the first statement. If $R=\mathbf{R}$ or $H^{2}\left(X, \mathscr{O}_{X}\right)=0$, the cycle class map $\mathrm{CH}_{1}\left(X_{C}\right) \otimes_{\mathbf{Z}} \mathbf{Q} \rightarrow \operatorname{Hdg}^{2 d-2}(X(C), \mathbf{Q}(d-1))$ is onto (we use the convention that all classes are Hodge if $\left.H^{2}\left(X, \mathscr{O}_{X}\right)=0\right)$ : by the Lefschetz principle one reduces to the case $C=\mathbf{C}$, for which see [Lew99, p. 91]. It follows, by a trace argument, that the torsion subgroup of the right-hand side of (5.9) is canonically isomorphic to Coker $\left(\mathrm{CH}_{1}(X) \rightarrow \operatorname{Hdg}_{G}^{2 d-2}(X(C), \mathbf{Z}(d-1))_{0}\right)$, as desired.

Remarks 5.3. (i) According to the generalised Bloch conjecture, the hypothesis that $\mathrm{CH}_{0}\left(X_{C^{\prime}}\right)$ is supported on a surface for every algebraically closed field $C^{\prime}$ containing $R$ should be equivalent to the vanishing of $H^{i}\left(X, \mathscr{O}_{X}\right)$ for all $i \geq 3$ (see [Voi14, Conjecture 1.11], [Jan94, §3], Voi02, Théorème 22.17]).

(ii) If $R$ has infinite transcendence degree over $\mathbf{Q}$ (for instance, if $R=\mathbf{R}$ ), the group $\mathrm{CH}_{0}\left(X_{C^{\prime}}\right)$ is supported on a surface for every algebraically closed field $C^{\prime}$ containing $R$ if and only if it is so for $C^{\prime}=C$ (by an argument known as decomposition of the diagonal, see [Blo80, Appendix to Lecture 1]).

(iii) As a consequence of Proposition [5.2, if $X$ is a real threefold such that $\mathrm{CH}_{0}\left(X_{\mathbf{C}}\right)$ is supported on a surface (for instance, a uniruled threefold), the defect of the real integral Hodge conjecture for $X$ is measured by the subgroup $H_{\mathrm{nr}}^{3}(X, \mathbf{Q} / \mathbf{Z}(2))_{0}$ of the unramified cohomology group $H_{\mathrm{nr}}^{3}(X, \mathbf{Q} / \mathbf{Z}(2))=H^{0}\left(X, \mathscr{H}_{X}^{3}(\mathbf{Q} / \mathbf{Z}(2))\right)$ consisting of those classes whose restriction to any real point of $X$ vanishes.

5.3. Torsion 1-cycles. Thanks to Proposition 5.2, we are now in a position to derive consequences of the real integral Hodge conjecture on the study of $\mathrm{CH}_{1}(X)_{\text {tors }}$.

If $X$ is a smooth, proper and irreducible variety of dimension $d$ over $R$, we denote by $\lambda: \mathrm{CH}_{1}(X)_{\text {tors }} \rightarrow H_{\text {ét }}^{2 d-3}(X, \mathbf{Q} / \mathbf{Z}(d-1))$ Bloch's Abel-Jacobi map. (See [Blo79] for its construction over an algebraically closed field; the construction goes through over a real closed field, as explained in [vH00b, Theorem 3.1].) The map $\lambda$ takes its 
values in the inverse image $H_{G}^{2 d-3}(X(C), \mathbf{Q} / \mathbf{Z}(d-1))_{0}$ of $H_{G}^{2 d-2}(X(C), \mathbf{Z}(d-1))_{0}$ by the boundary map

$$
H_{G}^{2 d-3}(X(C), \mathbf{Q} / \mathbf{Z}(d-1)) \rightarrow H_{G}^{2 d-2}(X(C), \mathbf{Z}(d-1))
$$

since the composition of $\lambda$ with this boundary map coincides with the equivariant cycle class map (see [CTSS83, Corollaire 1] and Theorem 1.18).

In the next statements, we denote by $\mathrm{cl}_{R}$ the two Borel-Haefliger cycle class maps $\operatorname{Pic}(X) \rightarrow H^{1}(X(R), \mathbf{Z} / 2 \mathbf{Z})$ and $\mathrm{CH}_{1}(X) \rightarrow H^{d-1}(X(R), \mathbf{Z} / 2 \mathbf{Z})$.

Theorem 5.4. Let $X$ be a smooth, proper and geometrically irreducible variety, of dimension d, over a real closed field $R$. Assume that $R=\mathbf{R}$ or $H^{2}\left(X, \mathscr{O}_{X}\right)=0$, and that $X$ satisfies the real integral Hodge conjecture for 1-cycles. Finally, assume that for every algebraically closed field $C^{\prime}$ containing $R$, the group $\mathrm{CH}_{0}\left(X_{C^{\prime}}\right)$ is supported on a surface.

(i) Bloch's Abel-Jacobi map induces a surjection

$$
\lambda: \mathrm{CH}_{1}(X)_{\text {tors }} \rightarrow H_{G}^{2 d-3}(X(C), \mathbf{Q} / \mathbf{Z}(d-1))_{0}
$$

(even an isomorphism if $d \leq 3$ ).

(ii) The subgroup $\mathrm{cl}_{R}\left(\mathrm{CH}_{1}(X)\left[2^{\infty}\right]\right)$ and the image of the map

$$
H_{G}^{2}(X(C), \mathbf{Z}(1)) \rightarrow H_{G}^{2}(X(R), \mathbf{Z}(1))=H^{1}(X(R), \mathbf{Z} / 2 \mathbf{Z})
$$

obtained by composing the restriction map and the decomposition (1.30) are exact orthogonal complements under the Poincaré duality pairing.

In view of Proposition 2.9 and Theorem 1.18, Theorem 5.4 (ii) has the following corollary. It is the twin of Theorem 3.16 (i); when $d=2$, the two are equivalent.

Corollary 5.5. Let $X$ be as in Theorem 5.4. If $H^{2}\left(X, \mathscr{O}_{X}\right)=0$, the subgroups $\mathrm{cl}_{R}\left(\mathrm{CH}_{1}(X)\left[2^{\infty}\right]\right)$ and $\mathrm{cl}_{R}(\mathrm{Pic}(X))$ are exact orthogonal complements under the Poincaré duality pairing.

Proof of Theorem 5.4. We start with (i). It is well known that $\lambda$ is injective if $d \leq 3$ (see CTSS83, Corollaire 1]). It is also a general fact that $\operatorname{Im}(\lambda)=$ $N^{d-2} H_{G}^{2 d-3}(X(C), \mathbf{Q} / \mathbf{Z}(d-1))$ (see [vH00b, Theorem 3.1]). These remarks, together with (5.8) and with the next lemma, imply (i).

Lemma 5.6. The inverse image of $N^{d-2} H_{G}^{2 d-2}(X(C), \mathbf{Z}(d-1))$ by the boundary map (5.10) is equal to $N^{d-2} H_{G}^{2 d-3}(X(C), \mathbf{Q} / \mathbf{Z}(d-1))$.

Proof. Let us denote by $A_{r}^{p, q}$ (resp., $B_{r}^{p, q}$ ) the term $E_{r}^{p, q}$ (resp., $E_{r}^{p, q+1}$ ) of the coniveau spectral sequence (5.1) associated with $M=\mathbf{Q} / \mathbf{Z}(d-1)$ (resp., $M=\mathbf{Z}(d-1))$. The short exact sequence $0 \rightarrow \mathbf{Z} \rightarrow \mathbf{Q} \rightarrow \mathbf{Q} / \mathbf{Z} \rightarrow 0$ induces a morphism of cohomology 
theories with supports $H_{G, Z(C)}^{*}(X(C), \mathbf{Q} / \mathbf{Z}(d-1)) \rightarrow H_{G, Z(C)}^{*+1}(X(C), \mathbf{Z}(d-1))$ in the sense of [CTHK97, §5.1] and, hence, a morphism of spectral sequences

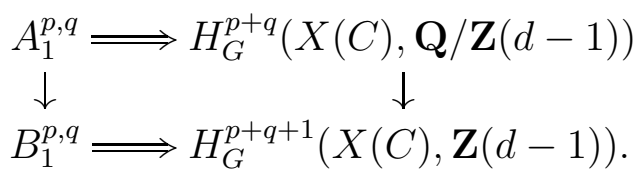

The sheaf $\mathscr{H}_{X}^{q}(\mathbf{Q}(d-1))$ vanishes for $q>d$, being a direct summand of $\pi_{*} \mathscr{H}_{X_{C}}^{q}(\mathbf{Q})$; hence, the boundary map $\mathscr{H}_{X}^{q}(\mathbf{Q} / \mathbf{Z}(d-1)) \rightarrow \mathscr{H}_{X}^{q+1}(\mathbf{Z}(d-1))$ is an isomorphism for $q>d$ and is surjective for $q=d$. We deduce, on the one hand, that $A_{2}^{p, q} \stackrel{\sim}{\longrightarrow} B_{2}^{p, q}$ for $q>d$ (thanks to (5.2) ), and, on the other hand, that the sequence

$$
0 \longrightarrow \mathscr{H}_{X}^{d}(\mathbf{Q} / \mathbf{Z}(d-1))_{0} \longrightarrow \mathscr{H}_{X}^{d}(\mathbf{Q} / \mathbf{Z}(d-1)) \longrightarrow \mathscr{H}_{X}^{d+1}(\mathbf{Z}(d-1)) \longrightarrow 0
$$

is exact (as we have already observed its exactness on the left, in (5.4)). Now, our hypothesis that $X$ satisfies the real integral Hodge conjecture for 1-cycles implies, by Proposition [5.2, that $H^{d-3}\left(X, \mathscr{H}_{X}^{d}(\mathbf{Q} / \mathbf{Z}(d-1))_{0}\right)=0$. It follows, in view of (5.12) and (5.2), that $A_{2}^{d-3, d} \hookrightarrow B_{2}^{d-3, d}$. Thus, the map $A_{2}^{p, q} \rightarrow B_{2}^{p, q}$ is injective for all $p, q$ such that $p+q=2 d-3$ and $q \geq d$. As the differentials $B_{r}^{p-r, q+r-1} \rightarrow B_{r}^{p, q}$ vanish for $r \geq 2$ and $q \geq d$ (indeed, even for $q \geq d+1-r$, see Proposition 5.1 (vi)), we conclude that $A_{\infty}^{p, q} \hookrightarrow B_{\infty}^{p, q}$ for all $p, q$ such that $p+q=2 d-3$ and $q \geq d$.

We now deduce (ii) from (i). By (i), by Theorem 1.18, and by the compatibility between $\lambda$ and the equivariant cycle class map (see [CTSS83, Corollaire 1]), we have

$$
\mathrm{cl}_{R}\left(\mathrm{CH}_{1}(X)\left[2^{\infty}\right]\right)=\mathrm{cl}_{R}\left(\mathrm{CH}_{1}(X)_{\text {tors }}\right)=\psi\left(\left(H_{G}^{2 d-2}(X(C), \mathbf{Z}(d-1))_{0}\right)_{\text {tors }}\right),
$$

where $\psi$ is as in Definition 3.2 .

From this point on, we proceed as in the proof of Theorem 3.3. Let us take up, from \$1.3.1, the notation $H^{p}=H^{p}(X(R), \mathbf{Z} / 2 \mathbf{Z})$. The map $u_{2}$ appearing in (1.46) can be inserted into a diagram

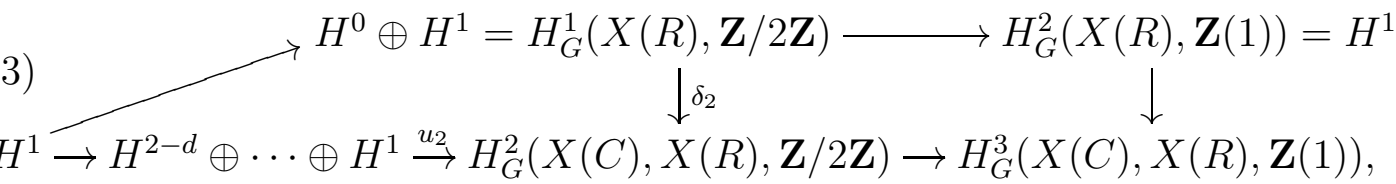

in which the leftmost arrows are the obvious inclusions, the vertical maps are the connecting homomorphisms of the localisation exact sequences, and the canonical isomorphisms of the first row are the decompositions (1.26) and (1.30). By the description of $u_{2}$ given after (1.46), this diagram commutes. In addition, the map from the bottom left $H^{1}$ to the top right $H^{1}$ is the identity map. It follows that the kernel of the composition $\theta_{1}^{\prime}: H^{1} \rightarrow H_{G}^{3}(X(C), X(R), \mathbf{Z}(1))$ of all of the maps 
of the bottom row of (5.13) coincides with the image of the map appearing in the statement of Theorem 5.4 (ii).

Let $\theta: H_{G}^{2 d-2}(X(C) \backslash X(R), \mathbf{Z}(d-1)) \rightarrow H^{d-1}$ denote the map defined at the end of the proof of Theorem 3.3 and let $\theta_{1}: H_{G}^{2 d-3}(X(C) \backslash X(R), \mathbf{Q} / \mathbf{Z}(d-1)) \rightarrow H^{d-1}$ denote its composition with the boundary map arising from the short exact sequence $0 \rightarrow \mathbf{Z} \rightarrow \mathbf{Q} \rightarrow \mathbf{Q} / \mathbf{Z} \rightarrow 0$. As remarked during the proof of Theorem 3.3, the map $\psi$ coincides with the composition of $\theta$ with the isomorphism of Lemma 3.5. Hence $\psi\left(\left(H_{G}^{2 d-2}(X(C), \mathbf{Z}(d-1))_{0}\right)\right.$ tors $)$ is equal to the image of $\theta_{1}$.

Unravelling the definitions of $\theta_{1}$ and of $\theta_{1}^{\prime}$ and applying Theorem 1.12, Proposition 1.10, and Remark 1.11 (ii), we see that the Pontrjagin dual of $\theta_{1}^{\prime}$ is $\theta_{1}$. The kernel of $\theta_{1}^{\prime}$ and the image of $\theta_{1}$ are therefore exact orthogonal complements, which completes the proof of (ii).

5.4. An example: torsion 1-cycles on real quartic threefolds. To illustrate the contents of \$5, we now determine the torsion subgroup of the Chow group of 1-cycles of a real quartic threefold with no real point.

Proposition 5.7. Let $R$ be a real closed field and $X \subset \mathbf{P}_{R}^{4}$ a smooth quartic threefold such that $X(R)=\varnothing$. If $X$ satisfies the real integral Hodge conjecture for 1-cycles, then there exists an isomorphism of abelian groups $\mathrm{CH}_{1}(X)_{\text {tors }} \simeq \mathbf{Z} / 2 \mathbf{Z} \oplus(\mathbf{Q} / \mathbf{Z})^{30}$.

We shall prove, in [BW18, $\S 7$, that if $R=\mathbf{R}$, then $X$ does satisfy the real integral Hodge conjecture for 1-cycles (an assertion which is equivalent to the existence of a geometrically irreducible curve of even geometric genus in $X$, by Theorem 3.22), so that the conclusion of Proposition 5.7 holds unconditionally in this case. We do not know whether the conclusion of Proposition 5.7 holds with no assumption on $R$.

Proof of Proposition 5.7. Applying [Roil72, Theorem 6] or the rational connectedness of Fano varieties shows that the group $\mathrm{CH}_{0}\left(X_{C^{\prime}}\right)$ is supported on a point for every algebraically closed field $C^{\prime}$ containing $R$. We can therefore apply Theorem 5.4 (i) and conclude that Bloch's Abel-Jacobi map induces an isomorphism $\mathrm{CH}_{1}(X)_{\text {tors }}=$ $H_{G}^{3}(X(C), \mathbf{Q} / \mathbf{Z}(2))$. The latter group is an extension of $H_{G}^{4}(X(C), \mathbf{Z}(2))_{\text {tors }}$ by $H_{G}^{3}(X(C), \mathbf{Z}(2)) \otimes_{\mathbf{Z}} \mathbf{Q} / \mathbf{Z}$. The next two lemmas now imply the proposition.

Lemma 5.8. There is a canonical isomorphism $H_{G}^{4}(X(C), \mathbf{Z}(2))=\mathbf{Z} \oplus \mathbf{Z} / 2 \mathbf{Z}$, the first summand being generated by $\operatorname{cl}(L+\bar{L})$, where $L \subset X_{C}$ denotes a line and $\bar{L}$ its conjugate.

Proof. The class $\operatorname{cl}(L+\bar{L}) \in H_{G}^{4}(X(C), \mathbf{Z}(2))$ generates a subgroup of index 2, by Proposition 3.14. In addition, this class is not divisible by 2 . Indeed, if it were, one would deduce, by taking the cup product with the class of $\mathscr{O}_{X}(1)$, the surjectivity of the natural map $H_{G}^{6}(X(C), \mathbf{Z}(3)) \rightarrow H^{6}(X(C), \mathbf{Z}(3))=\mathbf{Z}$, which would contradict Proposition 2.10 since $X(R)=\varnothing$. The lemma follows. 
Lemma 5.9. The finitely generated abelian group $H_{G}^{3}(X(C), \mathbf{Z}(2))$ has rank 30 .

Proof. Let us apply the Lefschetz fixed-point theorem to the complex conjugation involution of $X(C)$ (over an arbitrary real closed field, see [Bru92]). As this involution has no fixed point, we deduce from the canonical $G$-equivariant isomorphisms $H^{2 i}(X(C), \mathbf{Q})=\mathbf{Q}(-i)$ for $i \in\{0,1,2,3\}$ and from the vanishing of $H^{1}(X(C), \mathbf{Q})$ and of $H^{5}(X(C), \mathbf{Q})$ that the generator of $G$ acts on $H^{3}(X(C), \mathbf{Q})$ with trace 0 . On the other hand, the vector space $H^{3}(X(C), \mathbf{Q})$ has dimension 60 (see [EH16, Example 5.24]). This implies the lemma, since $H_{G}^{3}(X(C), \mathbf{Q})=H^{3}(X(C), \mathbf{Q})^{G}$.

Remarks 5.10. (i) When $R=\mathbf{R}$, one can exploit the structure of the group of real points of the intermediate Jacobian of $X$ to verify that in the situation of Proposition 5.7, Theorem 5.4 (i) allows one to produce, at the price of a significantly more involved computation, an isomorphism $\mathrm{CH}_{1}(X) \simeq \mathbf{Z} \oplus \mathbf{Z} / 2 \mathbf{Z} \oplus(\mathbf{R} / \mathbf{Z})^{30}$.

(ii) It is possible to perform the computations of Proposition 5.7 for some smooth quartic threefolds with real points. In this setting, Lemma 5.9 still holds, by Sil89, Chapter I, p. 12, (2.5)], and [BW18, Proposition 7.7] allows us to prove, when $R=\mathbf{R}$, that at least some of these varieties still satisfy the real integral Hodge conjecture. As an example, we have verified that $\mathrm{CH}_{1}(X)_{\text {tors }} \simeq \mathbf{Z} / 2 \mathbf{Z} \oplus(\mathbf{Q} / \mathbf{Z})^{30}$ if $X$ has homogeneous equation $x_{0}^{4}+x_{1}^{4}=x_{2}^{4}+x_{3}^{4}+x_{4}^{4}$.

\section{REFERENCES}

[AH61] M. F. Atiyah and F. Hirzebruch, Cohomologie-Operationen und charakteristische Klassen, Math. Z. 77 (1961), 149-187.

[AH62] Analytic cycles on complex manifolds, Topology 1 (1962), 25-45.

[AK85] S. Akbulut and H. King, Submanifolds and homology of nonsingular real algebraic varieties, Amer. J. Math. 107 (1985), no. 1, 45-83.

[AK88] _ Polynomial equations of immersed surfaces, Pacific J. Math. 131 (1988), no. 2, 209-217.

[AK93] _ Transcendental submanifolds of $\mathbf{R}^{n}$, Comment. Math. Helv. 68 (1993), no. 2, 308-318.

[AK03] C. Araujo and J. Kollár, Rational curves on varieties, Higher dimensional varieties and rational points (Budapest, 2001), Bolyai Soc. Math. Stud., vol. 12, Springer, Berlin, 2003, pp. 13-68.

[AP93] C. Allday and V. Puppe, Cohomological methods in transformation groups, Cambridge Studies in Advanced Mathematics, vol. 32, Cambridge University Press, Cambridge, 1993.

[BCC92] E. Ballico, F. Catanese and C. Ciliberto, Trento examples, Classification of irregular varieties (Trento, 1990), Lecture Notes in Math., vol. 1515, Springer, Berlin, 1992, pp. 134-139.

[BCR98] J. Bochnak, M. Coste and M.-F. Roy, Real algebraic geometry, Ergeb. Math. Grenzgeb. (3), vol. 36, Springer-Verlag, Berlin, 1998, translated from the 1987 French original, revised by the authors. 
[BD84] R. Benedetti and M. Dedò, Counterexamples to representing homology classes by real algebraic subvarieties up to homeomorphism, Compositio Math. 53 (1984), no. 2, 143151, erratum in 55, no. 3 (1985), p. 400.

[Ben16] O. Benoist, On Hilbert's 17th problem in low degree, preprint 2016, arXiv:1602.07330.

[Ber71] P. Berthelot, Quelques calculs de groupes $K$, Exp. IX, Théorie des intersections et théorème de Riemann-Roch, Séminaire de géométrie algébrique du Bois-Marie 19661967 (SGA 6), Lecture Notes in Mathematics, vol. 225, Springer-Verlag, Berlin, 1971.

[BH61] A. Borel and A. Haefliger, La classe d'homologie fondamentale d'un espace analytique, Bull. Soc. Math. France 89 (1961), 461-513.

[BK89] J. Bochnak and W. Kucharz, Algebraic models of smooth manifolds, Invent. math. 97 (1989), no. 3, 585-611.

[BK98] _ On homology classes represented by real algebraic varieties, Singularities Symposium - Łojasiewicz 70 (Kraków, 1996; Warsaw, 1996), Banach Center Publ., vol. 44, Polish Acad. Sci. Inst. Math., Warsaw, 1998, pp. 21-35.

[BK03] _ On approximation of smooth submanifolds by nonsingular real algebraic subvarieties, Ann. Sci. École Norm. Sup. (4) 36 (2003), no. 5, 685-690.

[Blo79] S. Bloch, Torsion algebraic cycles and a theorem of Roitman, Compositio Math. 39 (1979), no. 1, 107-127.

[Blo80] Lectures on algebraic cycles, Duke University Mathematics Series, IV, Duke University, Mathematics Department, Durham, N.C., 1980.

[BLR90] S. Bosch, W. Lütkebohmert and M. Raynaud, Néron models, Ergeb. Math. Grenzgeb. (3), vol. 21, Springer-Verlag, Berlin, 1990.

[BO74] S. Bloch and A. Ogus, Gersten's conjecture and the homology of schemes, Ann. Sci. École Norm. Sup. (4) 7 (1974), 181-201.

[BO18] O. Benoist and J. C. Ottem, Failure of the integral Hodge conjecture for threefolds of Kodaira dimension 0, arXiv:1802.01845, to appear in Comment. Math. Helv.

[Bre97] G. E. Bredon, Sheaf theory, second ed., Graduate Texts in Mathematics, vol. 170, Springer-Verlag, New York, 1997.

[Bro94] K. S. Brown, Cohomology of groups, Graduate Texts in Mathematics, vol. 87, SpringerVerlag, New York, 1994.

[Bru87] G. W. Brumfiel, Quotient spaces for semialgebraic equivalence relations, Math. Z. 195 (1987), no. 1, 69-78.

[Bru92] _ A Hopf fixed point theorem for semi-algebraic maps, Real algebraic geometry (Rennes, 1991), Lecture Notes in Math., vol. 1524, Springer, Berlin, 1992, pp. 163-169.

[BS58] A. Borel and J-P. Serre, Le théorème de Riemann-Roch, Bull. Soc. Math. France 86 (1958), 97-136.

[BS83] S. Bloch and V. Srinivas, Remarks on correspondences and algebraic cycles, Amer. J. Math. 105 (1983), no. 5, 1235-1253.

[BT82] R. Benedetti and A. Tognoli, Remarks and counterexamples in the theory of real algebraic vector bundles and cycles, Real algebraic geometry and quadratic forms (Rennes, 1981), Lecture Notes in Math., vol. 959, Springer, Berlin, 1982, pp. 198-211.

[BW18] O. Benoist and O. Wittenberg, On the integral Hodge conjecture for real varieties, II, preprint 2018.

[CT93] J.-L. Colliot-Thélène, Cycles algébriques de torsion et $K$-théorie algébrique, Arithmetic algebraic geometry (Trento, 1991), Lecture Notes in Math., vol. 1553, Springer, Berlin, 1993, pp. 1-49. 
[CTHK97] J.-L. Colliot-Thélène, R. T. Hoobler and B. Kahn, The Bloch-Ogus-Gabber theorem, Algebraic $K$-theory (Toronto, ON, 1996), Fields Inst. Commun., vol. 16, Amer. Math. Soc., Providence, RI, 1997, pp. 31-94.

[CTM04] J.-L. Colliot-Thélène and D. A. Madore, Surfaces de del Pezzo sans point rationnel sur un corps de dimension cohomologique un, J. Inst. Math. Jussieu 3 (2004), no. 1, 1-16.

[CTP90] J.-L. Colliot-Thélène and R. Parimala, Real components of algebraic varieties and étale cohomology, Invent. math. 101 (1990), no. 1, 81-99.

[CTS96] J.-L. Colliot-Thélène and C. Scheiderer, Zero-cycles and cohomology on real algebraic varieties, Topology 35 (1996), no. 2, 533-559.

[CTSS83] J.-L. Colliot-Thélène, J.-J. Sansuc and C. Soulé, Torsion dans le groupe de Chow de codimension deux, Duke Math. J. 50 (1983), no. 3, 763-801.

[CTV12] J.-L. Colliot-Thélène and C. Voisin, Cohomologie non ramifiée et conjecture de Hodge entière, Duke Math. J. 161 (2012), no. 5, 735-801.

[Del73] P. Deligne, La formule de dualité globale, Exp. XVIII, Théorie des topos et cohomologie étale des schémas, Séminaire de géométrie algébrique du Bois-Marie 1963-1964 (SGA 4), Tome 3, Lecture Notes in Mathematics, vol. 305, Springer-Verlag, Berlin, 1973.

[Del85] H. Delfs, The homotopy axiom in semialgebraic cohomology, J. reine angew. Math. 355 (1985), 108-128.

[Del91] Homology of locally semialgebraic spaces, Lecture Notes in Mathematics, vol. 1484, Springer-Verlag, Berlin, 1991.

[DK81a] H. Delfs and M. Knebusch, Semialgebraic topology over a real closed field I: Paths and components in the set of rational points of an algebraic variety, Math. Z. 177 (1981), no. $1,107-129$.

[DK81b] - Semialgebraic topology over a real closed field II: Basic theory of semialgebraic spaces, Math. Z. 178 (1981), no. 2, 175-213.

[DK82a] On the homology of algebraic varieties over real closed fields, J. reine angew. Math. 335 (1982), 122-163.

[DK82b] _ Semialgebraic topology over a real closed field, Ordered fields and real algebraic geometry (San Francisco, Calif., 1981), Contemp. Math., vol. 8, Amer. Math. Soc., Providence, R.I., 1982, pp. 61-78.

[DK84] _ An introduction to locally semialgebraic spaces, Rocky Mountain J. Math. 14 (1984), no. 4, 945-963, Ordered fields and real algebraic geometry (Boulder, Colo., 1983).

[DK85] L Locally semialgebraic spaces, Lecture Notes in Mathematics, vol. 1173, SpringerVerlag, Berlin, 1985.

[DK96] A. Degtyarev and V. Kharlamov, Topological classification of real Enriques surfaces, Topology 35 (1996), no. 3, 711-729.

[dSLF09] P. F. dos Santos and P. Lima-Filho, Bigraded equivariant cohomology of real quadrics, Adv. Math. 221 (2009), no. 4, 1247-1280.

[EH16] D. Eisenbud and J. Harris, 3264 and All That, A Second Course in Algebraic Geometry, Cambridge University Press, Cambridge, 2016.

[ELW15] H. Esnault, M. Levine and O. Wittenberg, Index of varieties over Henselian fields and Euler characteristic of coherent sheaves, J. Algebraic Geom. 24 (2015), no. 4, 693-718.

[EP14] M. J. Edmundo and L. Prelli, The six Grothendieck operations on o-minimal sheaves, preprint 2014, arXiv:1401.0846.

[Eps66] D. B. A. Epstein, Steenrod operations in homological algebra, Invent. math. 1 (1966), $152-208$. 
[Flo13] E. Floris, Fundamental divisors on Fano varieties of index $n-3$, Geom. Dedicata 162 (2013), 1-7.

[Ful98] W. Fulton, Intersection theory, second ed., Ergeb. Math. Grenzgeb. (3), vol. 2, SpringerVerlag, Berlin, 1998.

[Gey67] W.-D. Geyer, Ein algebraischer Beweis des Satzes von Weichold über reele algebraische Funktionenkörper, Algebraische Zahlentheorie (Ber. Tagung Math. Forschungsinst. Oberwolfach, 1964), Bibliographisches Institut, Mannheim, 1967, pp. 83-98.

[GH81] B. H. Gross and J. Harris, Real algebraic curves, Ann. Sci. École Norm. Sup. (4) 14 (1981), no. 2, 157-182.

[GH85] P. Griffiths and J. Harris, On the Noether-Lefschetz theorem and some remarks on codimension-two cycles, Math. Ann. 271 (1985), no. 1, 31-51.

[God49] L. Godeaux, Sur la construction de surfaces non rationnelles de genres zéro, Acad. Roy. Belgique. Bull. Cl. Sci. (5) 35 (1949), 688-693.

[Gra04] C. Grabowski, On the integral Hodge conjecture for 3-folds, Ph. D. thesis, Duke University, 2004.

[Gro57] A. Grothendieck, Sur quelques points d'algèbre homologique, Tôhoku Math. J. (2) 9 (1957), 119-221.

[Gro68] ㄴ Le groupe de Brauer I, II, III, Dix exposés sur la cohomologie des schémas, North-Holland, Amsterdam, 1968, pp. 46-188.

[Hau13] O. Haution, Degree formula for the Euler characteristic, Proc. Amer. Math. Soc. 141 (2013), no. 6, 1863-1869.

[Hir64] H. Hironaka, Resolution of singularities of an algebraic variety over a field of characteristic zero I, Ann. of Math. (2) 79 (1964), 109-203.

[Hir94] M. W. Hirsch, Differential topology, Graduate Texts in Mathematics, vol. 33, SpringerVerlag, New York, 1994, corrected reprint of the 1976 original.

[HV11] A. Höring and C. Voisin, Anticanonical divisors and curve classes on Fano manifolds, Pure Appl. Math. Q. 7 (2011), no. 4, 1371-1393.

[HV12] J. Heller and M. Voineagu, Equivariant semi-topological invariants, Atiyah's KR-theory, and real algebraic cycles, Trans. Amer. Math. Soc. 364 (2012), no. 12, 6565-6603.

[Jan94] U. Jannsen, Motivic sheaves and filtrations on Chow groups, Motives (Seattle, WA, 1991), Proc. Sympos. Pure Math., vol. 55, Amer. Math. Soc., Providence, RI, 1994, pp. 245-302.

[KA79] S. Kleiman and A. Altman, Bertini theorems for hypersurface sections containing a subscheme, Comm. Algebra 7 (1979), no. 8, 775-790.

[Kah87] B. Kahn, Construction de classes de Chern équivariantes pour un fibré vectoriel réel, Comm. Algebra 15 (1987), no. 4, 695-711.

[Kah93] _ Deux théorèmes de comparaison en cohomologie étale: applications, Duke Math. J. 69 (1993), no. 1, 137-165.

[Kar90] N. A. Karpenko, Algebro-geometric invariants of quadratic forms, Algebra i Analiz 2 (1990), no. 1, 141-162.

[Kat73] N. Katz, Etude cohomologique des pinceaux de Lefschetz, Exp. XVIII, Groupes de monodromie en géométrie algébrique, II, Séminaire de géométrie algébrique du BoisMarie 1967-1969 (SGA 7 II), Lecture Notes in Mathematics, vol. 340, Springer-Verlag, Berlin-New York, 1973.

[Kne76a] M. Knebusch, On algebraic curves over real closed fields. I, Math. Z. 150 (1976), no. 1, 49-70. 
[Kne76b] - On algebraic curves over real closed fields. II, Math. Z. 151 (1976), no. 2, 189-205.

[Kol13] J. Kollár, Esnault-Levine-Wittenberg indices, preprint 2013, arXiv:1312.3923.

[Kra91] V. A. Krasnov, Characteristic classes of vector bundles on a real algebraic variety, Izv. Akad. Nauk SSSR Ser. Mat. 55 (1991), no. 4, 716-746.

[Kra94]_. On the equivariant Grothendieck cohomology of a real algebraic variety and its application, Izv. Ross. Akad. Nauk Ser. Mat. 58 (1994), no. 3, 36-52.

[KS94] M. Kashiwara and P. Schapira, Sheaves on manifolds, Grundlehren der mathematischen Wissenschaften, vol. 292, Springer-Verlag, Berlin, 1994.

[Kuc85] W. Kucharz, On homology of real algebraic sets, Invent. math. 82 (1985), no. 1, 19-25.

[Kuc96] - Algebraic equivalence and homology classes of real algebraic cycles, Math. Nachr. 180 (1996), 135-140.

[Kuc01] - Algebraic equivalence of real divisors, Math. Z. 238 (2001), no. 4, 817-827.

[Lew99] J. D. Lewis, A survey of the Hodge conjecture, second ed., CRM Monograph Series, vol. 10, American Mathematical Society, Providence, RI, 1999.

[Lic69] S. Lichtenbaum, Duality theorems for curves over p-adic fields, Invent. math. 7 (1969), $120-136$.

[Man97] F. Mangolte, Cycles algébriques sur les surfaces K3 réelles, Math. Z. 225 (1997), no. 4, $559-576$.

[Man17] _ Variétés algébriques réelles, Cours spécialisés, vol. 24, Société Mathématique de France, Paris, 2017.

[Mil80] J. S. Milne, Étale cohomology, Princeton Mathematical Series, vol. 33, Princeton University Press, Princeton, N.J., 1980.

[MS74] J. W. Milnor and J. D. Stasheff, Characteristic classes, Princeton University Press, Princeton, N. J.; University of Tokyo Press, Tokyo, 1974, Annals of Mathematics Studies, No. 76.

[MvH98] F. Mangolte and J. van Hamel, Algebraic cycles and topology of real Enriques surfaces, Compositio Math. 110 (1998), no. 2, 215-237.

[Pan04] I. Panin, Riemann-Roch theorems for oriented cohomology, Axiomatic, enriched and motivic homotopy theory, NATO Sci. Ser. II Math. Phys. Chem., vol. 131, Kluwer Acad. Publ., Dordrecht, 2004, pp. 261-333.

[Pfi65] A. Pfister, Zur Darstellung von -1 als Summe von Quadraten in einem Körper, J. London Math. Soc. 40 (1965), 159-165.

[Ray68] Mme M. Raynaud, Modules projectifs universels, Invent. math. 6 (1968), 1-26.

[Rei79] M. Reid, Surfaces with $p_{g}=0, K^{2}=2$, preprint 1979, available from the author's webpage, http://homepages . warwick.ac.uk/ masda/surf/K2=2.pdf.

[Ris82] J.-J. Risler, Sur l'homologie des surfaces algébriques réelles, Real algebraic geometry and quadratic forms (Rennes, 1981), Lecture Notes in Math., vol. 959, Springer, Berlin-New York, 1982 , pp. 381-385.

[Rob83] R. Robson, Embedding semi-algebraic spaces, Math. Z. 183 (1983), no. 3, 365-370.

[Roĭ72] A. A. Roŭtman, Rational equivalence of 0-cycles, Mat. Sb. (N.S.) 89 (1972), 569-585.

[Sai89] S. Saito, A global duality theorem for varieties over global fields, Algebraic $K$-theory: connections with geometry and topology (Lake Louise, AB, 1987), NATO Adv. Sci. Inst. Ser. C Math. Phys. Sci., vol. 279, Kluwer Acad. Publ., Dordrecht, 1989, pp. 425-444.

[Sch94] C. Scheiderer, Real and étale cohomology, Lecture Notes in Mathematics, vol. 1588, Springer-Verlag, Berlin, 1994. 
[Sch95] - Purity theorems for real spectra and applications, Real analytic and algebraic geometry (Trento, 1992), de Gruyter, Berlin, 1995, pp. 229-250.

[Sch98] C. Schoen, An integral analog of the Tate conjecture for one-dimensional cycles on varieties over finite fields, Math. Ann. 311 (1998), no. 3, 493-500.

[Sch18] S. Schreieder, Stably irrational hypersurfaces of small slopes, preprint 2018, arXiv:1801.05397.

[Sil82] R. Silhol, $A$ bound on the order of $H_{n-1}^{(a)}(X, \mathbf{Z} / 2)$ on a real algebraic variety, Real algebraic geometry and quadratic forms (Rennes, 1981), Lecture Notes in Math., vol. 959, Springer, Berlin-New York, 1982, pp. 443-450.

[Sil89] _ Real algebraic surfaces, Lecture Notes in Mathematics, vol. 1392, SpringerVerlag, Berlin, 1989.

[SV05] C. Soulé and C. Voisin, Torsion cohomology classes and algebraic cycles on complex projective manifolds, Adv. Math. 198 (2005), no. 1, 107-127.

[Tei95] P. Teichner, 6-dimensional manifolds without totally algebraic homology, Proc. Amer. Math. Soc. 123 (1995), no. 9, 2909-2914.

[Tot13] B. Totaro, On the integral Hodge and Tate conjectures over a number field, Forum Math. Sigma 1 (2013), e4, 13 pages.

[vH00a] J. van Hamel, Algebraic cycles and topology of real algebraic varieties, CWI Tract, vol. 129, Stichting Mathematisch Centrum, Centrum voor Wiskunde en Informatica, Amsterdam, 2000, Dissertation, Vrije Universiteit Amsterdam, Amsterdam.

[vH00b] - Torsion zero-cycles and the Abel-Jacobi map over the real numbers, The arithmetic and geometry of algebraic cycles (Banff, AB, 1998), CRM Proc. Lecture Notes, vol. 24, Amer. Math. Soc., Providence, RI, 2000, pp. 329-359.

[Voi02] C. Voisin, Théorie de Hodge et géométrie algébrique complexe, Cours Spécialisés, vol. 10, Société Mathématique de France, Paris, 2002.

[Voi06] On integral Hodge classes on uniruled or Calabi-Yau threefolds, Moduli spaces and arithmetic geometry, Adv. Stud. Pure Math., vol. 45, Math. Soc. Japan, Tokyo, 2006, pp. 43-73.

[Voi07] _ Some aspects of the Hodge conjecture, Jpn. J. Math. 2 (2007), no. 2, 261-296.

[Voi13] L Remarks on curve classes on rationally connected varieties, A celebration of algebraic geometry, Clay Math. Proc., vol. 18, Amer. Math. Soc., Providence, RI, 2013, pp. 591-599.

[Voi14] Chow rings, decomposition of the diagonal, and the topology of families, Annals of Mathematics Studies, vol. 187, Princeton University Press, Princeton, NJ, 2014.

[Wit34] E. Witt, Zerlegung reeller algebraischer Funktionen in Quadrate. Schiefkörper über reellem Funktionenkörper, J. reine angew. Math. 171 (1934), 4-11.

Institut de Recherche Mathématique AvancÉe, UMR 7501, Université De StrasBourg et CNRS, 7 rue René Descartes, 67000 Strasbourg, FRANCE

E-mail address: olivier.benoist@unistra.fr

DÉPARTEMENT DE MATHÉMATIQUES ET APPLICATIONS, ÉCOLE NORMALE SUPÉRIEURE, 45 RUE D'Ulm, 75230 PARIS CEDEX 05, France

E-mail address: wittenberg@dma.ens.fr 